\title{
MULTIPLICATIVE PARAMETRIZED HOMOTOPY THEORY VIA SYMMETRIC SPECTRA IN RETRACTIVE SPACES
}

\author{
FABIAN HEBESTREIT ${ }^{1}$, STEFFEN SAGAVE ${ }^{2}$ and \\ CHRISTIAN SCHLICHTKRULL ${ }^{3}$ \\ ${ }^{1}$ Mathematical Institute, University of Bonn, Endenicher Allee 60, 53115 Bonn, Germany; \\ email: f.hebestreit@math.uni-bonn.de \\ 2 IMAPP, Radboud University Nijmegen, PO Box 9010, 6500 GL Nijmegen, The Netherlands; \\ email:s.sagave@math.ru.nl \\ ${ }^{3}$ Department of Mathematics, University of Bergen, P.O. Box 7803, 5020 Bergen, Norway; \\ email: christian.schlichtkrull@math.uib.no
}

Received 2 May 2019; accepted 28 January 2020

\begin{abstract}
In order to treat multiplicative phenomena in twisted (co)homology, we introduce a new point-setlevel framework for parametrized homotopy theory. We provide a convolution smash product that descends to the corresponding $\infty$-categorical product and allows for convenient constructions of commutative parametrized ring spectra. As an immediate application, we compare various models for generalized Thom spectra. In a companion paper, this approach is used to compare homotopical and operator algebraic models for twisted $K$-theory.
\end{abstract}

2010 Mathematics Subject Classification: 55P43 (primary); 55P42 (secondary)

\section{Introduction}

Stable parametrized homotopy theory originally arose from the study of transfer maps and fiberwise duality for generalized (co)homology theories. To analyze these phenomena, Clapp and Puppe [13] introduced a first homotopy category of parametrized spectra. Later, May and Sigurdsson [37] studied a more refined model category of orthogonal parametrized spectra over a base space $B$ enjoying favorable point-set topological properties. Both these approaches relate duality to

(c) The Author(s) 2020. This is an Open Access article, distributed under the terms of the Creative Commons Attribution-NonCommercialShareAlike licence (http://creativecommons.org/licenses/by-nc-sa/4.0/), which permits noncommercial re-use, distribution, and reproduction in any medium, provided the same Creative Commons licence is included and the original work is properly cited. The written permission of Cambridge University Press must be obtained for commercial re-use. 
a smash product obtained by first forming an external fiberwise smash product lying over $B \times B$ and then internalizing it by pullback along the diagonal $B \rightarrow$ $B \times B$.

When studying cross and cup products in twisted (co)homology through the representing parametrized spectra, one needs a different symmetric monoidal structure. Suppose that the base space $B$ has a homotopy coherent commutative multiplication, that is, an $E_{\infty}$ structure. Then one can also attempt to internalize the external fiberwise smash product over $B \times B$ by pushout along the multiplication $\mu: B \times B \rightarrow B$. We will refer to this type of product as a convolution smash product to distinguish it from the fiberwise smash product considered above. However, the setup of May-Sigurdsson does not provide such a symmetric monoidal convolution smash product unless the multiplication of $B$ is strictly associative and commutative, ruling out the parameter spaces of many interesting parametrized spectra, such as those representing twisted $K$-theory or bordism theories. Ando et al. [1] implemented the convolution smash product in the $\infty$-categorical setup from [2] and showed how it, for example, gives rise to twisted Umkehr maps.

The primary aim of the present paper is to introduce a convenient point-set-level category of parametrized spectra that admits a symmetric monoidal convolution smash product descending to the $\infty$-categorical product of [1]. Our main new idea is to also allow the base space to vary for the different levels of a parametrized spectrum. More precisely, the base spaces will assemble to an $\mathcal{I}$-space, that is, a functor from the category $\mathcal{I}$ of finite sets $\mathbf{n}=\{1, \ldots, n\}, n \geqslant 0$, and injective maps to the category of spaces $\mathcal{S}$. Replacing the cartesian product of base spaces, the category $\mathcal{S}^{\mathcal{I}}$ of $\mathcal{I}$-spaces is equipped with a symmetric monoidal Day convolution product $\otimes$ induced by the concatenation in $\mathcal{I}$ and the cartesian product of spaces. We call commutative monoids with respect to $\nabla$ commutative $\mathcal{I}$-space monoids.

It is proved in [42] that every $E_{\infty}$ homotopy type arises as the homotopy colimit $M_{h \mathcal{I}}=\operatorname{hocolim}_{\mathcal{I}} M$ for a commutative $\mathcal{I}$-space monoid $M$. We think of $M_{h \mathcal{I}}$ as the underlying $E_{\infty}$ space of $M$. This point of view often leads to simple and explicit models of $E_{\infty}$ spaces. Working with symmetric spectra parametrized over commutative $\mathcal{I}$-space monoids allows us to implement the notion of a convolution smash product in a convenient fashion. Different pointset frameworks for parametrized homotopy theory were recently developed, for example, in $[11,19]$, but, to our knowledge, these approaches again do not allow for a convolution smash product in sufficient generality for the applications we have in mind.

In a companion paper [21], the first named authors use the setup developed here to prove that twisted $K$-theory as defined via operator algebraic methods 
coincides with the version defined via homotopy theoretic methods. More specifically, it is shown that these theories agree as commutative parametrized ring spectra with respect to the convolution smash product. In this case, the commutative $\mathcal{I}$-space monoids serving as base spaces model the classifying space of the projective orthogonal group of a Hilbert space.

1.1. Symmetric spectra in retractive spaces. To implement our approach, we first consider the category $\mathcal{S}_{\mathcal{R}}$ of retractive spaces. Objects of $\mathcal{S}_{\mathcal{R}}$ are pairs of spaces $(U, K)$ with structure maps $K \rightarrow U \rightarrow K$ that compose to the identity. Morphisms in $\mathcal{S}_{\mathcal{R}}$ are pairs of maps making the two obvious squares commutative. The external fiberwise smash product $\bar{\wedge}$ provides a symmetric monoidal structure on $\mathcal{S}_{\mathcal{R}}$ with unit $\left(S^{0}, *\right)$. On base spaces, this $\bar{\wedge}$-product is just the cartesian product.

Following the work of Hovey [26], we form the category $\mathrm{Sp}_{\mathcal{R}}^{\Sigma}$ of symmetric spectrum objects in $\mathcal{S}_{\mathcal{R}}$ with $-\bar{\wedge}\left(S^{1}, *\right)$ as the suspension functor. It comes with a local model category structure whose fibrant objects are $\Omega$-spectra. (We avoid the term stable since in lack of a zero object, $\operatorname{Ho}\left(\operatorname{Sp}_{\mathcal{R}}^{\Sigma}\right)$ is not stable in the technical sense.) The category $\mathrm{Sp}_{\mathcal{R}}^{\Sigma}$ inherits a symmetric monoidal product $\bar{\wedge}$ from $\mathcal{S}_{\mathcal{R}}$ that will play the role of the external smash product. An object $(E, X)$ of $\operatorname{Sp}_{\mathcal{R}}^{\Sigma}$ is a sequence of retractive spaces $\left(E_{n}, X_{n}\right)$ with an action of the symmetric group $\Sigma_{n}$ and structure maps $\left(E_{n}, X_{n}\right) \bar{\wedge}\left(S^{1}, *\right) \rightarrow\left(E_{n+1}, X_{n+1}\right)$ compatible with the $\Sigma_{n}$-actions. Inspecting definitions, the projection $\pi_{b}: \mathcal{S}_{\mathcal{R}} \rightarrow \mathcal{S}$ to the base space induces a projection $\pi_{b}: \mathrm{Sp}_{\mathcal{R}}^{\Sigma} \rightarrow \mathcal{S}^{\mathcal{I}}$ to base $\mathcal{I}$-spaces. If $X$ is an $\mathcal{I}$-space, we define the category of $X$-relative symmetric spectra $\operatorname{Sp}_{X}^{\Sigma}$ to be the fiber of $\pi_{b}$ over $X$. We stress that unless $X$ is constant, $\mathrm{Sp}_{X}^{\Sigma}$ is not the category of symmetric spectrum objects in some base category since the levels of $(E, X)$ take values in different categories.

A map of $\mathcal{I}$-spaces $f: X \rightarrow Y$ induces an adjunction $f_{!}: \operatorname{Sp}_{X}^{\Sigma} \rightleftarrows \operatorname{Sp}_{Y}^{\Sigma}: f^{*}$ where $f^{*}$ denotes degreewise pullback. We say that $f$ is an $\mathcal{I}$-equivalence if the map of homotopy colimits $f_{h \mathcal{I}}: X_{h \mathcal{I}} \rightarrow Y_{h \mathcal{I}}$ is a weak homotopy equivalence and note that the $\mathcal{I}$-equivalences are the weak equivalences in an $\mathcal{I}$-model structure on $\mathcal{S}^{\mathcal{I}}$ (see [42]).

THEOREM 1.2. Let $X$ be an $\mathcal{I}$-space. The category $\operatorname{Sp}_{X}^{\Sigma}$ of $X$-relative symmetric spectra admits a local model structure where a map is a cofibration, fibration, or weak equivalence if and only if it is so as a map in $\mathrm{Sp}_{\mathcal{R}}^{\Sigma}$.

With respect to the local model structure, $\left(f_{!}, f^{*}\right)$ is a Quillen adjunction that is a Quillen equivalence if $f$ is an $\mathcal{I}$-equivalence. In particular, $\mathrm{Sp}_{X}^{\Sigma}$ is Quillen equivalent to the stabilization of the category of spaces over and under $X_{h \mathcal{I}}$. 
Consequently, $\mathrm{Sp}_{X}^{\Sigma}$ models the same homotopy theory as the category of $X_{h \mathcal{I}^{-}}$ parametrized spectra in the sense of May-Sigurdsson [37] or [2]. We also show that $\mathbb{R} f^{*}: \operatorname{Ho}\left(\operatorname{Sp}_{Y}^{\Sigma}\right) \rightarrow \operatorname{Ho}\left(\operatorname{Sp}_{X}^{\Sigma}\right)$ admits a right adjoint $\mathbb{R} f_{*}$ that does, however, not arise from a right Quillen functor.

We do, in fact, provide two versions of the local model structure in the theorem, an absolute and a positive one, where as usual the positive version is necessary to lift the model structures to categories of commutative monoids (see (1.4)). The theorem also has a much easier unstable analogue: the category of retractive spaces $\mathcal{S}_{\mathcal{R}}$ inherits a model structure from the category of spaces where a map is a weak equivalence if both of its components are, and the standard model structure on the category of spaces $\mathcal{S}_{K}$ over and under $K$ can be viewed as a 'restriction' of this model structure to the subcategory $\mathcal{S}_{K}$ of $\mathcal{S}_{\mathcal{R}}$. However, the proof of Theorem 1.2 turns out to be not as easy as it may look. The problem is that the factorizations needed for the model category structure on $\operatorname{Sp}_{X}^{\Sigma}$ are not inherited from $\operatorname{Sp}_{\mathcal{R}}^{\Sigma}$ since the factorizations in the latter category may change the base object. To circumvent this problem, we give an intrinsic description of the category $\mathrm{Sp}_{X}^{\Sigma}$ and its local model structure in terms of section categories and then show that its cofibrations, fibrations, and weak equivalences are detected in $\mathrm{Sp}_{\mathcal{R}}^{\Sigma}$.

It is also useful to note that the model category $\operatorname{Sp}_{\mathcal{R}}^{\Sigma}$ can be recovered from the $\operatorname{Sp}_{X}^{\Sigma}$ for varying $X$. As a category, $\mathrm{Sp}_{\mathcal{R}}^{\Sigma}$ is equivalent to the Grothendieck construction of the pseudofunctor $X \mapsto \operatorname{Sp}_{X}^{\Sigma}$ sending an $\mathcal{I}$-space $X$ to $\operatorname{Sp}_{X}^{\Sigma}$ and a map $f: X \rightarrow Y$ to $f_{!}$. Harpaz and Prasma [20] have identified conditions under which a model structure on the base category and model structures on the values of a pseudofunctor assemble to a so-called integral model structure on the Grothendieck construction, and we verify these conditions in the case at hand.

THEOREM 1.3. The $\mathcal{I}$-model structure on $\mathcal{S}^{\mathcal{I}}$ and the local model structures on the $\mathrm{Sp}_{X}^{\Sigma}$ induce an integral model structure on the Grothendieck construction of $X \mapsto \mathrm{Sp}_{X}^{\Sigma}$. Under the equivalence of the Grothendieck construction with $\mathrm{Sp}_{\mathcal{R}}^{\Sigma}$, the integral model structure corresponds to the local model structure on $\mathrm{Sp}_{\mathcal{R}}^{\Sigma}$.

This theorem is again analogous to the unstable situation where it is easy to check that $\mathcal{S}_{\mathcal{R}}$ is equivalent to the Grothendieck construction of the pseudofunctor $K \mapsto \mathcal{S}_{K}$ on $\mathcal{S}$ and that the integral model structure exists and is equivalent to the model structure on $\mathcal{S}_{\mathcal{R}}$ considered earlier.

The fiberwise smash product on $\mathcal{S}_{\mathcal{R}}$ induces a fiberwise smash product $\bar{\wedge}$ on $\mathrm{Sp}_{\mathcal{R}}^{\Sigma}$ that is the $\otimes$-product on the base $\mathcal{I}$-spaces. Therefore, it restricts to an external fiberwise smash product $\operatorname{Sp}_{X}^{\Sigma} \times \mathrm{Sp}_{X}^{\Sigma} \rightarrow \mathrm{Sp}_{X \bowtie X}^{\Sigma}$. For a commutative $\mathcal{I}$-space monoid $M$, we can now use the pushforward along the multiplication 
$\mu: M \otimes M \rightarrow M$ to define a symmetric monoidal convolution product

$$
\mathrm{Sp}_{M}^{\Sigma} \times \mathrm{Sp}_{M}^{\Sigma} \stackrel{\bar{\top}}{\rightarrow} \mathrm{Sp}_{M \otimes M}^{\Sigma} \stackrel{\mu_{!}}{\rightarrow} \mathrm{Sp}_{M}^{\Sigma}
$$

on the category of $M$-relative symmetric spectra.

Now any parametrized spectrum $(E, X) \in \mathrm{Sp}_{\mathcal{R}}^{\Sigma}$ gives rise to a parametrized (co)homology theory as expected. To define it, recall that there is an $\mathcal{I}$ spacification functor $\mathcal{S} / X_{h \mathcal{I}} \rightarrow \mathcal{S}^{\mathcal{I}} / X, \tau \mapsto \tau_{\mathcal{I}}$ that is homotopy inverse to $\operatorname{hocolim}_{\mathcal{I}}[45$, Section 4]. We then set

$$
\begin{aligned}
& (E, X)_{n}: \mathcal{S} / X_{h \mathcal{I}} \longrightarrow \mathrm{Ab}, \quad\left(\tau: A \rightarrow X_{h \mathcal{I}}\right) \longmapsto \pi_{n}(\mathbb{L} \Theta)\left(\mathbb{R} \tau_{\mathcal{I}}^{*}\right)(E, X) \quad \text { and } \\
& (E, X)^{n}: \mathcal{S} / X_{h \mathcal{I}} \longrightarrow \mathrm{Ab}, \quad\left(\tau: A \rightarrow X_{h \mathcal{I}}\right) \longmapsto \pi_{-n}(\mathbb{R} \Gamma)\left(\mathbb{R} \tau_{\mathcal{I}}^{*}\right)(E, X),
\end{aligned}
$$

where, for any $\mathcal{I}$-space $Y$, the functors $\mathbb{L} \Theta, \mathbb{R} \Gamma: \operatorname{Ho}\left(\operatorname{Sp}_{Y}^{\Sigma}\right) \rightarrow \operatorname{Ho}\left(\operatorname{Sp}^{\Sigma}\right)$ denote the left and right adjoint, respectively, of the derived pullback functor along the unique map $Y \rightarrow *$.

Let $(R, M)$ be a parametrized ring spectrum, that is, a monoid object in $\operatorname{Sp}_{\mathcal{R}}^{\Sigma}$. Then we obtain the cross product displayed as the upper horizontal arrow in the following square:

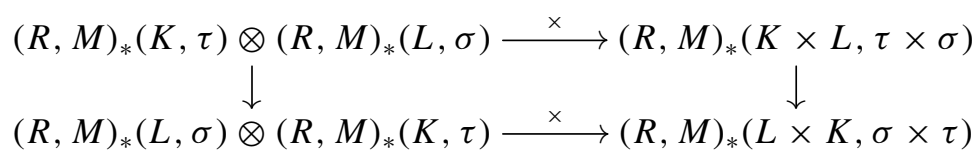

When $(R, M)$ is commutative, the square commutes up to the usual sign where the right-hand vertical map is induced by the essentially unique homotopy between the following two maps arising from the $E_{\infty}$-structure on $M_{h \mathcal{I}}$ :

$$
\begin{gathered}
L \times K \stackrel{\mathrm{tw}}{\rightarrow} K \times L \stackrel{\tau \times \sigma}{\rightarrow} M_{h \mathcal{I}} \times M_{h \mathcal{I}} \stackrel{\mu}{\rightarrow} M_{h \mathcal{I}} \\
L \times K \stackrel{\sigma \times \tau}{\longrightarrow} M_{h \mathcal{I}} \times M_{h \mathcal{I}} \stackrel{\mu}{\rightarrow} M_{h \mathcal{I}}
\end{gathered}
$$

An analogous statement holds for the cup and cross products in cohomology.

1.5. Comparison to the $\infty$-categorical setup. We can also use Theorem 1.3 to compare the categories $\operatorname{Sp}_{X}^{\Sigma}$ to the $\infty$-categorical setup of parametrized homotopy theory. There the category of parametrized spectra over a space $K$ is given by $\operatorname{Fun}\left(K, \mathrm{Sp}_{\infty}\right)$, and these categories also assemble into a category of parametrized spectra with varying base space by Lurie's higher categorical version of the Grothendieck construction. The resulting category is also known as the tangent category $T \mathcal{S}_{\infty}$ of the $\infty$-category of spaces $\mathcal{S}_{\infty}$, and we shall adopt this name to ease notation in the comparison results. 
TheOREM 1.6. For an $\mathcal{I}$-space $X$, the underlying $\infty$-category of the local model structure on $\operatorname{Sp}_{X}^{\Sigma}$ is canonically equivalent to $\operatorname{Fun}\left(X_{h \mathcal{I}}, \mathrm{Sp}_{\infty}\right)$, which translates the Quillen adjunction $\left(f_{!}, f^{*}\right)$ for any map $f: X \rightarrow X^{\prime}$ into its $\infty$ categorical counterpart. Therefore, as $X$ varies, these equivalences assemble into an equivalence between $\left(\operatorname{Sp}_{\mathcal{R}}^{\Sigma}\right)_{\infty}$ and $T \mathcal{S}_{\infty}$.

Furthermore, this equivalence is symmetric monoidal with respect to the exterior smash product on both sides, and for $M$ a commutative $\mathcal{I}$-space monoid, the equivalence $\operatorname{Sp}_{M}^{\Sigma} \simeq \operatorname{Fun}\left(M_{h \mathcal{I}}, \mathrm{Sp}_{\infty}\right)$ is symmetric monoidal for the convolution smash product on the left and Day convolution on the right.

In fact, as far as we know, the symmetric monoidal structure on $T \mathcal{S}_{\infty}$ has not appeared in the literature before. Therefore, extending the recent work of Nikolaus [38], we provide the necessary material on the stabilization of fibrations of $\infty$-operads needed to construct it, which may be of independent interest.

\subsection{Universal bundles and Thom spectra. When $R$ is a (sufficiently fibrant)} commutative symmetric ring spectrum, then its underlying multiplicative infinite loop space and its units arise as commutative $\mathcal{I}$-space monoids $\mathrm{GL}_{1}^{\mathcal{I}} R \subset \Omega^{\mathcal{I}}(R)$ (see [44]). By definition, we have $\Omega^{\mathcal{I}}(R)(\mathbf{n})=\Omega^{n} R_{n}$ with structure maps and multiplication maps induced by those of $R$, and $\mathrm{GL}_{1}^{\mathcal{I}} R$ is the subobject whose path components represent units in $\pi_{0}(R)$. The underlying $E_{\infty}$ space of $\mathrm{GL}_{1}^{\mathcal{I}} R$ is a model of what is usually denoted by $\mathrm{GL}_{1} R$. Writing $G$ for (a suitable cofibrant replacement of) $\mathrm{GL}_{1}^{\mathcal{I}} R$, the inclusion $G \rightarrow \Omega^{\mathcal{I}}(R)$ is adjoint to a map of commutative symmetric ring spectra $\mathbb{S}^{\mathcal{I}}[G] \rightarrow R$ from the spherical monoid ring of $G$.

There also is a parametrized suspension spectrum $\mathbb{S}_{t}^{\mathcal{I}}[G]$ of $G$ with $G$ as base commutative $\mathcal{I}$-space monoid. The above map $\mathbb{S}^{\mathcal{I}}[G] \rightarrow R$ and the projection to the terminal $\mathcal{I}$-space $G \rightarrow *$ induce commutative $\mathbb{S}_{t}^{\mathcal{I}}[G]$-algebra structures on $\mathbb{S}$ and $R$ that allow us to form the two-sided bar construction $B^{\bar{\lambda}}\left(\mathbb{S}, \mathbb{S}_{t}^{\mathcal{I}}[G], R\right)$ in commutative parametrized ring spectra. Its base commutative $\mathcal{I}$-space monoid is the bar construction $B G$ of $G$ with respect to $\bigotimes$ which models the infinite loop space $B \mathrm{GL}_{1} R$. We write $\gamma_{R}$ for (a suitable fibrant replacement of) $B^{\bar{\lambda}}(\mathbb{S}$, $\left.\mathbb{S}_{t}^{\mathcal{I}}[G], R\right)$ and view this $B G$-relative commutative symmetric ring spectrum as the universal $R$-line bundle over $B G$. It only depends on the stable equivalence type of $R$ and is mapped to the $\infty$-categorical version of the universal $R$-line bundle from [1] under the equivalence from Theorem 1.6 (including multiplicative structures). Moreover, $\gamma_{R}$ gives rise to an $R$-module Thom spectrum functor

$$
T_{\mathcal{R}}^{\mathcal{I}}: \mathcal{S}^{\mathcal{I}} / B G \rightarrow \operatorname{Mod}_{R}, \quad(f: X \rightarrow B G) \mapsto(X \rightarrow *) !\left(f^{*} \gamma_{R}\right)
$$

on $\mathcal{I}$-spaces over $B G$ that is homotopy invariant and lax symmetric monoidal. 
Precomposing with the $\mathcal{I}$-spacification $\mathcal{S} /(B G)_{h \mathcal{I}} \rightarrow \mathcal{S}^{\mathcal{I}} / B G$ mentioned above, we also get a Thom spectrum functor $T_{\mathcal{R}}: \mathcal{S} /(B G)_{h \mathcal{I}} \rightarrow \operatorname{Mod}_{R}$ defined on maps of spaces to $(B G)_{h \mathcal{I}} \simeq B \mathrm{GL}_{1} R$.

The functor $T_{\mathcal{R}}^{\mathcal{I}}$ provides a different construction of the $R$-module Thom spectra studied by Ando et al. [2,3] making the underlying parametrized spectra explicit on the point-set level. We give the following multiplicative comparison of these two and the multiplicative Thom spectrum functors studied by Basu et al. [6].

THEOREM 1.8. The Thom spectrum functor $T_{\mathcal{R}}$ defined in terms of parametrized spectra, the R-module Thom spectrum functor $T_{E G}$ from [6], and the Thom spectrum functor from [2] are all equivalent, and the equivalences respect the monoidal structures and the operad actions preserved by these functors.

Over the sphere spectrum $\mathbb{S}$, we provide a new interpretation of the 'classical' Thom spectrum functor considered in $[33,45]$ : Let $F(\mathbf{n})$ denote the topological monoid of basepoint preserving homotopy equivalences of $S^{n}$. Letting $\mathbf{n}$ vary, we show that the usual one-sided bar construction on $F(\mathbf{n})$ gives rise to a commutative parametrized symmetric ring spectrum $B^{\times}(*, F, \mathbb{S})$ that is locally equivalent to the universal line bundle for $\mathbb{S}$. Using this, we get an explicit multiplicative equivalence relating the classical description of the Thom spectrum functor to the parametrized approach in the present paper.

\subsection{Homotopical and operator algebraic models for twisted $\boldsymbol{K}$-theory. As} mentioned, in a companion paper [21], the first two authors use the framework developed here to relate operator algebraic models for various twisted $K$-theory spectra to their homotopical counterparts defined in terms of pullbacks of the universal bundle just discussed. To obtain the comparison, there we generalize the construction of $B^{\times}(*, F, \mathbb{S})$ by considering actions on symmetric ring spectra of what we term cartesian $\mathcal{I}$-monoids, that is, $\mathcal{I}$-diagrams in topological monoids. For such an action of $H$ on $R$, one can produce a homotopy quotient spectrum $R / / H \in \mathrm{Sp}_{B^{\times}(H)}^{\Sigma}$, where $B^{\times}(H)$ denotes the bar construction of $H$ with respect to the cartesian product on $\mathcal{S}^{\mathcal{I}}$. In particular, $B^{\times}(*, F, \mathbb{S})=\mathbb{S} / / F$. When $H$ is grouplike and $R$ and $H$ are fibrant, we establish a comparison between $R / / H$ and the pullback of $\gamma_{R}$ along a certain map $B H \rightarrow B \mathrm{GL}_{1}^{\mathcal{I}}(R)$ induced by evaluating the action of $H$ on the unit of $R$ [21, Proposition 4.2]. When $R, H$, and the action are suitably commutative, then this comparison is one of the commutative parametrized ring spectra.

The whole construction can then be applied to actions of the cartesian $\mathcal{I}$ monoid $\mathrm{PO}$ formed by the projective orthogonal groups of the Hilbert spaces $L^{2}\left(\mathbb{R}^{n}\right)$ on the $K$-theory spectra introduced by Joachim in [28], and this action 
satisfies the commutativity assumptions mentioned above. The spectrum $\mathrm{KO} / / \mathrm{PO}$ is easily related to operator algebraic definitions of twisted $K$-theory, whereas by the comparison results we produce here, the pullback of $\gamma_{\mathrm{KO}}$ is an incarnation of the homotopy theoretic definition. This allows us to deduce the equivalence of the usual definitions of twisted $K$-theory as considered by operator algebraists and homotopy theorists. Furthermore, we describe the resulting map $B \mathrm{PO} \rightarrow$ $B \mathrm{GL}_{1}^{\mathcal{I}} \mathrm{KO}$ in purely homotopical terms, completing partial results by Antieau et al. [4].

1.10. Organization. In Section 2, we recall the category of retractive spaces and describe the relevant features of its model structure. In Section 3, we then introduce our categories of parametrized spectra and their level model structures both in the absolute setting and relative to an $\mathcal{I}$-space. After discussing the external fiberwise and convolution smash products in the short Section 4, we establish the local model structures in Section 5. In Section 6, we compare the different local model structures, prove Theorems 1.2 and 1.3, and show how the positive local model structures lift to commutative parametrized ring spectra. Section 7 is about the (co)homology theories associated with parametrized spectra. In Section 8, we introduce the universal line bundle. Section 9 is about Thom spectra and provides the proof of Theorem 1.8. Section 10 compares our constructions to the $\infty$-categorical approach and provides the proof of Theorem 1.6.

1.11. Notations and conventions. We write $\mathcal{S}$ for the category of spaces, which can either be the category of compactly generated weak Hausdorff spaces Top or the category of simplicial sets sSet. We will only distinguish between the simplicial and the topological case when arguments differ. Moreover, we freely use the language of model categories and refer to [23] as our primary source.

\section{Retractive spaces}

In this section, we collect basic results about retractive spaces. This material is mostly easy and, for example, treated in [11, Section 1.1]. We carry out some details for later reference and to fix notations.

DEFINITION 2.1. A retractive space is a pair of spaces $(U, K)$ with structure maps $K \rightarrow U$ and $U \rightarrow K$ that compose to the identity of $K$. A map of retractive spaces $(U, K) \rightarrow(V, L)$ is a pair of maps $U \rightarrow V$ and $K \rightarrow L$ such that the two 
squares in

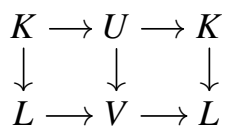

commute. We refer to $U$ as the total space of the retractive space $(U, K)$, call $K$ its base space, and write $\mathcal{S}_{\mathcal{R}}$ for the category of retractive spaces.

Construction 2.2. Spaces and retractive spaces are related in various ways. The following diagram summarizes the constructions relevant for us:
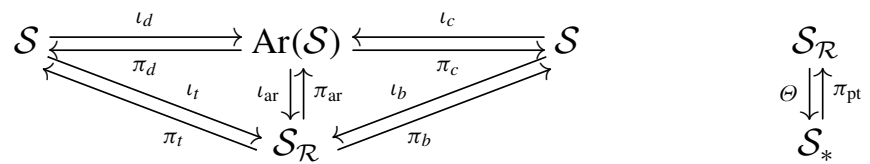

Here $\operatorname{Ar}(\mathcal{S})$ is the arrow category of $\mathcal{S}, \pi_{d}(V \rightarrow L)=V$ and $\pi_{c}(V \rightarrow L)=L$ are the forgetful functors projecting to the domain and codomain, and $\iota_{c}(K)=$ $(\emptyset \rightarrow K)$ and $\iota_{d}(K)=\mathrm{id}_{K}$ are their left adjoints. The forgetful functor $\pi_{\mathrm{ar}}(V, L)=(V \rightarrow L)$ remembers only the projection to the base and has a left adjoint $\iota_{\mathrm{ar}}(f: A \rightarrow B)=(B \amalg A, B)$ with structure maps given by incl $_{B}: B \rightarrow B \amalg A$ and $\left(\mathrm{id}_{B}, f\right): B \amalg A \rightarrow B$. Consequently, we obtain composite functors $\pi_{b}(V, L)=L$ and $\pi_{t}(V, L)=V$ projecting to base and total space. Their left adjoints are given by $\iota_{b}(K)=(K, K)$ and $\iota_{t}(K)=(K \amalg K, K)$. A based space $T$ can be viewed as a retractive space $(T, *)=(* \rightarrow T \rightarrow *)$ that we often denote by $T$. The functor $\pi_{\mathrm{pt}}: \mathcal{S}_{*} \rightarrow \mathcal{S}_{\mathcal{R}}, T \mapsto T$ is right adjoint to $\Theta(U, K)=U / K$.

LEMMA 2.3. The functor $\iota_{b}: \mathcal{S} \rightarrow \mathcal{S}_{\mathcal{R}}$ is both left and right adjoint to $\pi_{b}$.

The category of retractive spaces $\mathcal{S}_{\mathcal{R}}$ is complete and cocomplete since it can be viewed as a category of functors with values in $\mathcal{S}$. The last lemma implies that both $\iota_{b}$ and $\pi_{b}$ preserve limits and colimits.

2.4. Retractive spaces as a model category. In the following, we use the standard model structures on $\mathcal{S}=$ sSet and $\mathcal{S}=$ Top with weak equivalences the weak homotopy equivalences. We say that a map of retractive spaces $(U, K) \rightarrow(V, L)$ is

- a weak equivalence if both $K \rightarrow L$ and $U \rightarrow V$ are weak equivalences in $\mathcal{S}$,

- a cofibration if both $K \rightarrow L$ and $U \cup_{K} L \rightarrow V$ are cofibrations in $\mathcal{S}$, and

- a fibration if both $K \rightarrow L$ and $U \rightarrow V \times_{L} K$ are fibrations in $\mathcal{S}$. 
Proposition 2.5. These classes of maps provide a model structure on $\mathcal{S}_{\mathcal{R}}$.

Proof. This follows from standard model category arguments. Alternatively, one can identify $\mathcal{S}_{\mathcal{R}}$ with the diagrams in $\mathcal{S}$ indexed by a Reedy category with two objects $B$ and $T$, one nonidentity degree raising morphism $s: B \rightarrow T$, one nonidentity degree lowering morphism $r: T \rightarrow B$, and one nonidentity endomorphism $s r$ of $T$. With this, the proposition follows from the general theory of Reedy model structures (see, for example, [23, Theorem 15.3.4]).

To establish more properties of the model category $\mathcal{S}_{\mathcal{R}}$, we note that the following lifting properties hold.

Lemma 2.6. Let $i: A \rightarrow B$ and $(U, K) \rightarrow(V, L)$ be maps in $\mathcal{S}$ and $\mathcal{S}_{\mathcal{R}}$, respectively.

(i) The maps $i: A \rightarrow B$ and $K \rightarrow L$ have the lifting property in $\mathcal{S}$ if and only if the maps $\iota_{b}(i: A \rightarrow B)=((A, A) \rightarrow(B, B))$ and $(U, K) \rightarrow(V, L)$ have the lifting property in $\mathcal{S}_{\mathcal{R}}$.

(ii) The maps $i: A \rightarrow B$ and $U \rightarrow V \times{ }_{L} K$ have the lifting property in $\mathcal{S}$ if and only if $\iota_{\mathrm{ar}}\left(i \rightarrow \mathrm{id}_{B}\right)=((B \amalg A, B) \rightarrow(B \amalg B, B))$ and $(U, K) \rightarrow(V, L)$ have the lifting property in $\mathcal{S}_{\mathcal{R}}$.

If $S$ is a set of maps in $\mathcal{S}$, we write $S_{\mathcal{R}}$ for the set

$$
\left\{\iota_{b}(i) \mid i \in S\right\} \cup\left\{\iota_{\mathrm{ar}}\left(i \rightarrow \mathrm{id}_{B}\right) \mid i: A \rightarrow B \in S\right\}
$$

of maps in $\mathcal{S}_{\mathcal{R}}$. Moreover, we let $I$ and $J$ be the standard sets of generating cofibrations and generating acyclic cofibrations for $\mathcal{S}$.

PROPOSITION 2.7. The model category $\mathcal{S}_{\mathcal{R}}$ is cofibrantly generated with generating cofibrations $I_{\mathcal{R}}$ and generating acyclic cofibrations $J_{\mathcal{R}}$.

We refer to [23, Definition 12.1.1] or [26, Appendix A] for the notion of a cellular model category. This property is useful because left proper cellular model categories admit left Bousfield localizations [23, Theorem 4.1.1].

Proposition 2.8. The model category $\mathcal{S}_{\mathcal{R}}$ is proper and cellular.

Proof. It is easy to see that the properness of $\mathcal{S}_{\mathcal{R}}$ is inherited from $\mathcal{S}$. Cellularity is inherited since $\mathcal{S}$ is cellular and the projection $\mathcal{S}_{\mathcal{R}} \rightarrow \mathcal{S} \times \mathcal{S}$ which forgets the structure maps preserves and detects colimits and limits and sends cofibrations in $\mathcal{S}_{\mathcal{R}}$ to objectwise cofibrations in $\mathcal{S} \times \mathcal{S}$. 
2.9. Retractive spaces as a Grothendieck construction. Recall that

$$
\pi_{b}: \mathcal{S}_{\mathcal{R}} \rightarrow \mathcal{S}, \quad(U, K) \mapsto K
$$

denotes the projection to the base space. Let $\mathcal{S}_{K}$ be the fiber of $\pi_{b}$ over a space $K$, that is, the subcategory of $\mathcal{S}_{\mathcal{R}}$ whose objects have $K$ as the base space and whose morphisms are the identity on the base. The category $\mathcal{S}_{K}$ is equivalent to the category of spaces over and under $K$, that is, to the category of pointed objects in the over-category $\mathcal{S} / K$. Other common notations for $\mathcal{S}_{K}$ are $(\mathcal{S} / K)_{*}$ or $\mathcal{S}_{K}^{K}$.

Every map of spaces $f: K \rightarrow L$ induces an adjoint pair of functors

$$
f_{!}: \mathcal{S}_{K} \rightleftarrows \mathcal{S}_{L}: f^{*}
$$

The left adjoint sends $(U, K)$ to $\left(U \cup_{K} L, L\right)$ with its canonical structure maps, and the right adjoint sends $(V, L)$ to $\left(f^{*}(V), K\right)$ with its canonical structure maps.

We write Cat for the 'category' of (not necessarily small) categories. Recall that a pseudofunctor $\mathcal{C}: \mathcal{K} \rightarrow$ Cat on a category $\mathcal{K}$ consists of categories $\mathcal{C}_{K}$ for every object $K$ of $\mathcal{K}$ and functors $\alpha_{!}: \mathcal{C}_{K} \rightarrow \mathcal{C}_{L}$ for every morphism $\alpha: K \rightarrow L$ of $\mathcal{K}$. The condition that $\mathcal{C}_{-}$is a pseudofunctor (rather than a functor) amounts to saying that there are coherent isomorphisms (rather than identities) $\mathrm{id}_{\mathcal{C}_{K}} \cong$ $\left(\mathrm{id}_{K}\right)_{!}$and $g_{!} f_{!} \cong(g f)$ ! for composable morphisms $f$ and $g$ in $\mathcal{K}$. We refer to [8, Definition 7.5.1] for a complete definition.

The universal property of the pushout implies:

LEMMA 2.12. The categories $\mathcal{S}_{K}$ assemble to a pseudofunctor $\mathcal{S} \rightarrow$ Cat given by

$$
K \mapsto \mathcal{S}_{K}, \quad(f: K \rightarrow L) \mapsto\left(f_{!}: \mathcal{S}_{K} \rightarrow \mathcal{S}_{L}\right)
$$

The Grothendieck construction of a pseudofunctor $F: \mathcal{K} \rightarrow$ Cat is the category $\mathcal{K} \int F$ with objects the pairs $(K, U)$ with $K \in \mathrm{Ob} \mathcal{K}$ and $U \in \mathrm{Ob} F(K)$. Morphisms $(K, U) \rightarrow(L, V)$ are pairs of morphisms $f: K \rightarrow L$ in $\mathcal{K}$ and $F(f)(U) \rightarrow V$ in $F(L)$, and the composite is defined in the obvious way [50, Definition 3.1.2].

LEMMA 2.14. The Grothendieck construction of (2.13) is equivalent to $\mathcal{S}_{\mathcal{R}}$.

Proof. If $(U, K) \rightarrow(V, L)$ is a morphism in $\mathcal{S}_{\mathcal{R}}$, then its map of total spaces factors as $U \rightarrow(K \rightarrow L) !(U) \rightarrow V$ where the second map is over and under $L$. 
REMARK 2.15. Equivalently, one checks that the projection (2.10) is a (cartesian) fibration; see, for example, [9, Definition 8.1.2 and Theorem 8.3.1].

We equip the categories $\mathcal{S}_{K}$ with the standard model structures where a map is a cofibration, fibration, or weak equivalence if the map of total spaces has this property in $\mathcal{S}$. With these model structures, the categories $\mathcal{S}_{K}$ are cofibrantly generated, cellular, and proper [24]. The following homotopical properties of the adjunction (2.11) easily follow from the properness of $\mathcal{S}$ :

LEMMA 2.16. Let $f: K \rightarrow$ L be a map of spaces.

(i) The adjunction $\left(f_{!}, f^{*}\right)$ is a Quillen adjunction.

(ii) If $f$ is a weak equivalence, then $\left(f_{!}, f^{*}\right)$ is a Quillen equivalence.

(iii) If $f$ is an acyclic cofibration, then $f_{\text {! }}$ preserves weak equivalences.

(iv) If $f$ is an acyclic fibration, then $f^{*}$ preserves weak equivalences.

Next we recall the terminology of [20, Definition 3.0.4].

Definition 2.17. Let $\mathcal{K}$ be a model category and let $F: \mathcal{K} \rightarrow$ Cat be a pseudofunctor such that each $F(K)$ is equipped with a model structure and such that $F$ maps each morphism $f: K \rightarrow L$ in $\mathcal{K}$ to a left Quillen functor $F(f): F(K) \rightarrow F(L)$. We write $f_{\text {! }}$ for $F(f)$ and $f^{*}$ for its right adjoint. We say that a morphism in $\mathcal{K} \int F$ consisting of $f: K \rightarrow L$ and $f_{!}(U) \rightarrow V$ is

- an integral cofibration if $f$ and $f_{!}(U) \rightarrow V$ are cofibrations,

- an integral fibration if $f$ and $U \rightarrow f^{*}(V)$ are fibrations, and

- an integral weak equivalence if $f$ is a weak equivalence and for any cofibrant replacement $U^{\text {cof }} \rightarrow U$ in $F(K)$, the composite of $f_{!}\left(U^{\text {cof }}\right) \rightarrow f_{!}(U) \rightarrow V$ is a weak equivalence.

It is shown in [20, Theorem 3.0.12] that these classes of maps form a model structure when $F$ is a proper relative pseudofunctor (in the language of [20, Section 3]). This amounts to requiring that the conditions of Lemma 2.16 hold for $F$. Applying this discussion to the pseudofunctor (2.13) thus provides the following statement.

Proposition 2.18. These classes form a model structure on the Grothendieck construction of $K \mapsto \mathcal{S}_{K}$, called the integral model structure. Under the 
equivalence of Lemma 2.14, it coincides with the model structure on $\mathcal{S}_{\mathcal{R}}$ considered earlier.

2.19. The monoidal structure on retractive spaces. Let $(U, K)$ and $(V, L)$ be retractive spaces. Their structure maps induce a commutative diagram

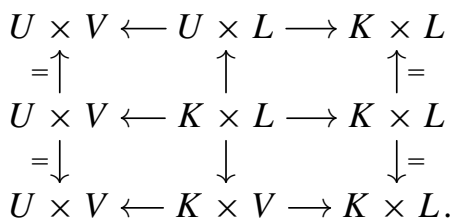

DEFINITION 2.21. Let $U \bar{\wedge} V$ be the colimit of (2.20). It is the total space of the fiberwise smash product $(U, K) \bar{\wedge}(V, L)=(U \bar{\wedge} V, K \times L)$ of $(U, K)$ and $(V, L)$. Its structure maps are induced by the universal property of the pushout and the structure maps of $(U, K)$ and $(V, L)$. (We stress that we use the symbol $\bar{\wedge}$ both for the fiberwise smash product in $\mathcal{S}_{\mathcal{R}}$ and for its total space.)

Equivalently, $U \bar{\wedge} V$ is the iterated pushout obtained by first forming the vertical pushouts in (2.20) and then forming the pushout of the resulting diagram

$$
U \times V \leftarrow\left((U \times L) \cup_{K \times L}(K \times V)\right) \rightarrow K \times L .
$$

Writing $W^{V}$ for the cotensor in $\mathcal{S}$, there is an internal Hom-functor

$$
\operatorname{Hom}_{\mathcal{R}}: \mathcal{S}_{\mathcal{R}}^{\mathrm{op}} \times \mathcal{S}_{\mathcal{R}} \rightarrow \mathcal{S}_{\mathcal{R}}, \quad((V, L),(W, M)) \mapsto\left(W^{V} \times{ }_{\left(W^{L} \times_{M^{L}} M^{V}\right)} M^{L}, M^{L}\right),
$$

where the latter pair of spaces has the obvious structure maps making it an object of $\mathcal{S}_{\mathcal{R}}$.

PROPOSITION 2.24. The fiberwise smash product is a closed symmetric monoidal product on the category of retractive spaces $\mathcal{S}_{\mathcal{R}}$ with monoidal unit $S^{0}$. In particular, there is a natural isomorphism

$$
\mathcal{S}_{\mathcal{R}}((U, K) \bar{\wedge}(V, L),(W, M)) \cong \mathcal{S}_{\mathcal{R}}\left((U, K), \operatorname{Hom}_{\mathcal{R}}((V, L),(W, M))\right) .
$$

Proof. It is clear that the fiberwise smash product is symmetric monoidal. To establish the adjunction isomorphism (2.25), we note that on both sides, a morphism is given by a pair of maps $U \times V \rightarrow W$ and $K \times L \rightarrow M$ in $\mathcal{S}$ such that the following three diagrams commute: 


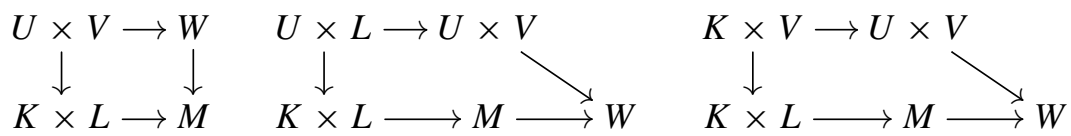

REMARK 2.26. The Hom-object considered here is an 'external' one in that the base space of $\operatorname{Hom}_{\mathcal{R}}((V, L),(W, L))$ is $L^{L}$ and not $L$. It does not appear to give rise to an 'internal' Hom-object in the category $\mathcal{S}_{L}$. While the latter 'internal' Hom is not relevant for our work, it plays an important role in the approach by May-Sigurdsson. Implementing it requires them to deal with considerably more involved point-set topological issues (see, for example, [37, Section 1.3]).

For later reference, we describe some $\bar{\wedge}$-products of retractive spaces considered so far. In the notation of Construction 2.2 and Lemma 2.6, we have

$$
\begin{aligned}
(A, A) & \wedge(V, L) \\
(B \amalg A, B) \bar{\wedge}(V, L) & \cong\left((B \times L, A \times L) \amalg_{(A \times L)}(A \times V), B \times L\right) \\
(B \amalg A, B) \bar{\wedge}\left(B^{\prime} \amalg A^{\prime}, B^{\prime}\right) & \cong\left(\left(B \times B^{\prime}\right) \amalg\left(A \times A^{\prime}\right),\left(B \times B^{\prime}\right)\right)
\end{aligned}
$$

PROPOSITION 2.27. The model category $\mathcal{S}_{\mathcal{R}}$ satisfies the pushout product axiom.

Proof. By [48, Lemma 3.5(i)], it suffices to verify the pushout product axiom for the generating (acyclic) cofibrations $I_{\mathcal{R}}$ and $J_{\mathcal{R}}$ in Proposition 2.7. Using the above isomorphisms (2.26), (2.27), and (2.28), the claim follows from the pushout product axiom for $\mathcal{S}$.

LEMMA 2.28. Let $(U, K)$ and $(V, L)$ be retractive spaces, and let $f: K \rightarrow K^{\prime}$ and $g: L \rightarrow L^{\prime}$ be maps in $\mathcal{S}$. Then there is a natural isomorphism

$$
\left(f_{!}(U), K^{\prime}\right) \bar{\wedge}\left(g_{!}(V), L^{\prime}\right) \stackrel{\cong}{\rightrightarrows}\left((f \times g)_{!}(U \bar{\wedge} V), K^{\prime} \times L^{\prime}\right) .
$$

Proof. The products $f_{!}(U) \times g_{!}(V)$ and $K^{\prime} \times L^{\prime}$ can be identified with the colimits of the following diagrams:

$$
\begin{aligned}
& K^{\prime} \times V \leftarrow K^{\prime} \times L \rightarrow K^{\prime} \times L^{\prime} \quad K^{\prime} \times L \leftarrow K^{\prime} \times L \rightarrow K^{\prime} \times L^{\prime}
\end{aligned}
$$

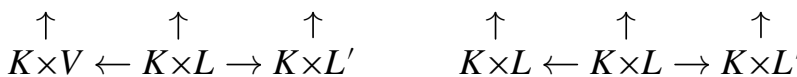

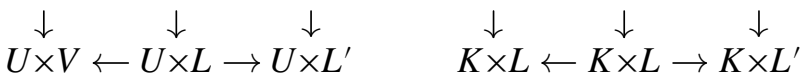

Moreover, $\left(f_{!}(U) \times L^{\prime}\right) \cup_{K^{\prime} \times L^{\prime}}\left(K^{\prime} \times g_{!}(V)\right)$ is isomorphic to the colimit of the following diagram: 


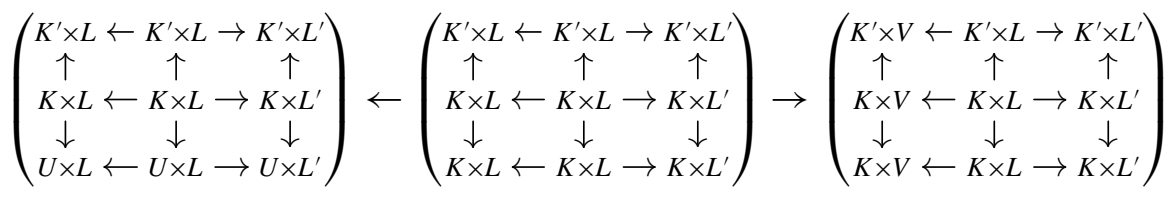

Hence, taking the colimit of each of the five $3 \times 3$-diagrams and then forming the iterated colimit as in (2.22) provides $f_{!}(U) \bar{\wedge} g_{!}(V)$. Since colimits commute among each other, we may alternatively first form the colimit as in (2.22) in each of the nine entries of the $3 \times 3$-diagrams and then form the colimit of the resulting $3 \times 3$-diagram:

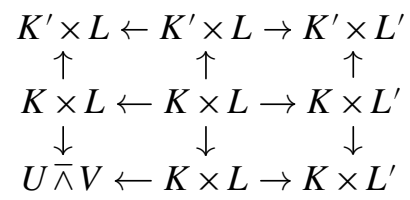

The colimit of the latter diagram is isomorphic to $(f \times g)_{!}(U \bar{\wedge} V)$.

The fiberwise smash product also commutes with base change.

Lemma 2.29. Let $(U, K)$ and $(V, L)$ be retractive spaces, and let $f: K^{\prime} \rightarrow K$ and $g: L^{\prime} \rightarrow L$ be maps in $\mathcal{S}$. Then there is a natural isomorphism

$$
\left(f^{*} U, K^{\prime}\right) \bar{\wedge}\left(g^{*} V, L^{\prime}\right) \rightarrow\left((f \times g)^{*}(U \bar{\wedge} V), K^{\prime} \times L^{\prime}\right) .
$$

Proof. It is clear that $(f \times g)^{*}(U \times V) \cong f^{*}(U) \times g^{*}(V)$. Moreover, as a functor between the underlying categories of sets, $(f \times g)^{*}$ preserves the pushouts that are used to form the fiberwise smash products. This shows the claim if $\mathcal{S}=$ sSet.

When $\mathcal{S}=$ Top, the argument is more involved since forming colimits in compactly generated weak Hausdorff spaces may change the underlying sets. By [32, Proposition 1.3], base change along a map of compactly generated weak Hausdorff spaces preserves colimits in compactly generated spaces. The pushout in compactly generated spaces of a diagram of compactly generated weak Hausdorff spaces in which one map is a closed inclusion coincides with its pushout in compactly generated weak Hausdorff spaces, and the cobase change of the closed inclusion is again a closed inclusion in this case [30, Appendix A, Proposition 7.5]. The structure map from the base to the total space of a retractive space (in compactly generated weak Hausdorff spaces) is a closed inclusion [37, Lemma 1.6.2] and closed inclusions are preserved under products. Hence, the pushout defining $(U \times L) \cup_{K \times L}(K \times V)$ is preserved under base change. Because $K \rightarrow U$ and $L \rightarrow V$ are closed inclusions, so is $(U \times L) \cup_{K \times L}(K \times V) \rightarrow U \times V$, and it follows from the description in (2.22) that the pushout defining $U \wedge V$ is preserved under base change. 
REMARK 2.31. Given retractive spaces $(U, K)$ and $(L, V)$ and points $x \in K$ and $y \in L$, the proposition shows that the fiber of $U \bar{\wedge} V \rightarrow K \times L$ over $(x, y)$ is isomorphic to the smash product of the fiber of $U \rightarrow K$ over $x$ with the fiber of $V \rightarrow L$ over $y$. This justifies the name fiberwise smash product.

2.32. Simplicial structure of $\mathcal{S}_{K}$. The symmetric monoidal structure on $\mathcal{S}_{\mathcal{R}}$ can be used to define simplicial and pointed simplicial structures on the category $\mathcal{S}_{K}$ for a fixed space $K$. If $Q$ is an unbased simplicial set, we define a functor

$$
Q \otimes-: \mathcal{S}_{K} \rightarrow \mathcal{S}_{K}, \quad(U, K) \mapsto\left(Q_{+}, *\right) \bar{\wedge}(U, K) .
$$

Here we implicitly compose with the cobase change along $K \times\{*\} \stackrel{\cong}{\rightarrow} K$ and apply the geometric realization to $Q$ when working with topological spaces.

Proposition 2.33. This action equips $\mathcal{S}_{K}$ with the structure of a simplicial model category.

Proof. An application of [17, Lemma II.2.4] shows that $\mathcal{S}_{K}$ becomes a simplicial category. The compatibility with the model structure follows from Proposition 2.27 and the compatibility of the model structures on $\mathcal{S}_{K}$ and $\mathcal{S}_{\mathcal{R}}$.

Since $\mathcal{S}_{K}$ is pointed, its simplicial structure induces a pointed simplicial structure. The tensor of $(U, K)$ with a pointed simplicial set $P$ is the pushout of $(K, K) \leftarrow\{*\} \otimes(U, K) \rightarrow P \otimes(U, K)$. It follows that this tensor is $(P, *) \bar{\wedge}(U, K)$. Consequently, the cotensor is $\operatorname{Hom}_{\mathcal{R}}((P, *),(U, K))$, and we deduce from (2.23) that it has the total space $U^{P} \times_{\left(U \times K^{P}\right)} K$. So a point in the total space consists of a map $h: P \rightarrow U$ whose image is contained in a single fiber and which sends the basepoint of $P$ to the canonical basepoint of the fiber.

\section{Twisted symmetric spectra}

We will now introduce a generalized form of symmetric spectra for which we allow the individual levels of a symmetric spectrum $X$ to take values in different categories. We will also construct level model structures on these categories that we will use in Section 5 to build the local model structures we are really after. Both the level and the local model structures come in an absolute and a positive version with different cofibrations. The positive version will be needed to get a lifted model structure on commutative parametrized ring spectra (see Section 6.16).

Let $\mathcal{I}$ be the category with objects the finite sets $\mathbf{m}=\{1, \ldots, m\}, m \geqslant 0$, and morphisms the injections. The ordered concatenation $\mathbf{m} \sqcup \mathbf{n}=\mathbf{m}+\mathbf{n}$ of finite 
sets makes $\mathcal{I}$ a symmetric strict monoidal category with unit $\mathbf{0}$. Its symmetry isomorphism is the shuffle $\chi_{m, n}: \mathbf{m} \sqcup \mathbf{n} \rightarrow \mathbf{n} \sqcup \mathbf{m}$ moving the first $m$ elements past the last $n$ elements.

We first recall some notions needed for a description of symmetric spectra using the category $\mathcal{I}$ (see, for example, [45, Section 3.1]). This viewpoint will be convenient for the discussion of convolution products in Section 4. Given a finite set $P$, we let $S^{P}=\bigwedge_{P} S^{1}$ be the $P$ fold smash power of $S^{1}$. If $\alpha: \mathbf{m} \rightarrow \mathbf{n}$ is a morphism in $\mathcal{I}$, we write $\mathbf{n}-\alpha$ for the complement of its image. The canonical extension of $\alpha$ to a bijection $\mathbf{m} \amalg(\mathbf{n}-\alpha) \rightarrow \mathbf{n}$ induces a homeomorphism

$$
S^{\mathbf{m}} \wedge S^{\mathbf{n}-\alpha} \stackrel{\cong}{\rightarrow} S^{\mathbf{n}} .
$$

More generally, if $\alpha: \mathbf{m} \rightarrow \mathbf{n}$ and $\beta: \mathbf{n} \rightarrow \mathbf{p}$ are composable morphisms in $\mathcal{I}$, then the canonical bijection $(\mathbf{n}-\alpha) \amalg(\mathbf{p}-\beta) \rightarrow \mathbf{p}-\beta \alpha$ induces a homeomorphism

$$
S^{\mathbf{n}-\alpha} \wedge S^{\mathbf{p}-\beta} \stackrel{\cong}{\rightarrow} S^{\mathbf{p}-\beta \alpha} \text {. }
$$

3.3. Quillen $\mathcal{I}$-categories. The next definition again uses the language of pseudofunctors [8, Definition 7.5.1] with values in Cat.

Definition 3.4. A Quillen $\mathcal{I}$-category is a pseudofunctor $\mathcal{C}: \mathcal{I} \rightarrow$ Cat with each $\mathcal{C}_{\mathbf{m}}$ equipped with a cofibrantly generated model structure and each $\alpha_{!}: \mathcal{C}_{\mathbf{m}} \rightarrow \mathcal{C}_{\mathbf{n}}$ left Quillen.

REMARK 3.5. This notion of a Quillen $\mathcal{I}$-category corresponds to the 'right Quillen presheaves' of [5, Definition 2.21] given by contravariant pseudofunctors on $\mathcal{I}$ that send maps to right Quillen functors. We use the present terminology since our examples below make it more natural to treat the left adjoints as primary data.

DefinITION 3.6. Let $\mathcal{C}: \mathcal{I} \rightarrow$ Cat be a Quillen $\mathcal{I}$-category. Its section category $\mathcal{C}^{\mathcal{I}}$ has as objects $X$ families of objects $X_{\mathbf{m}}$ in $\mathcal{C}_{\mathbf{m}}$ equipped with structure maps $\alpha_{!} X_{\mathbf{m}} \rightarrow X_{\mathbf{n}}$ for every $\alpha: \mathbf{m} \rightarrow \mathbf{n}$ in $\mathcal{I}$ such that the structure map $\left(\mathrm{id}_{\mathbf{m}}\right)_{!} X_{\mathbf{m}} \rightarrow$ $X_{\mathrm{m}}$ is the isomorphism given by the pseudofunctor and such that the square

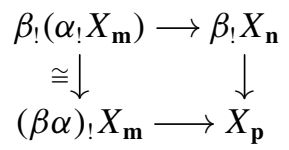

commutes for all maps $\alpha: \mathbf{m} \rightarrow \mathbf{n}$ and $\beta: \mathbf{n} \rightarrow \mathbf{p}$ in $\mathcal{I}$. Here the left-hand vertical map is the coherence isomorphism of the pseudofunctor $\mathcal{C}$, while the 
three other maps are the structure maps associated with $\alpha, \beta$, and $\beta \alpha$. Morphisms $X \rightarrow Y$ in $\mathcal{C}^{\mathcal{I}}$ are families of morphisms $X_{\mathbf{m}} \rightarrow Y_{\mathbf{m}}$ that make the obvious squares commutative.

If $\mathcal{C}: \mathcal{I} \rightarrow$ Cat is a Quillen $\mathcal{I}$-category and $\mathbf{m}$ is an object in $\mathcal{I}$, we get an adjunction

$$
F_{\mathbf{m}}: \mathcal{C}_{\mathbf{m}} \rightleftarrows \mathcal{C}^{\mathcal{I}}: \mathrm{Ev}_{\mathbf{m}}
$$

with right adjoint the evaluation functor sending $X$ in $\mathcal{C}^{\mathcal{I}}$ to $X_{\mathbf{m}}$. The left adjoint is given in level $\mathbf{n}$ by

$$
\left(F_{\mathbf{m}}(Z)\right)_{\mathbf{n}}=\coprod_{\alpha: \mathbf{m} \rightarrow \mathbf{n}} \alpha ! Z,
$$

where the coproduct is indexed over $\mathcal{I}(\mathbf{m}, \mathbf{n})$ and formed in $\mathcal{C}_{\mathbf{n}}$. The composition in $\mathcal{I}$ induces structure maps turning the $\left(F_{\mathbf{m}}(Z)\right)_{\mathbf{n}}$ into an object of $\mathcal{C}^{\mathcal{I}}$.

3.9. Level model structures. Let $f: X \rightarrow Y$ be a morphism in the section category of a Quillen $\mathcal{I}$-category. Then $f$ is an absolute level fibration (respectively level equivalence) if $f_{\mathbf{m}}: X_{\mathbf{m}} \rightarrow Y_{\mathbf{n}}$ is a fibration (respectively weak equivalence) in $\mathcal{C}_{\mathbf{m}}$ for all $\mathbf{m}$. Positive level fibrations and level weak equivalences are defined by only requiring this condition if $|\mathbf{m}| \geqslant 1$. The absolute (respectively positive) level cofibrations are the maps with the left lifting properties against all maps that are both absolute (respectively positive) level fibrations and level equivalences. The next statement is analogous to [5, Theorem 2.28].

Proposition 3.10. Let $\mathcal{C}: \mathcal{I} \rightarrow$ Cat be a Quillen $\mathcal{I}$-category. Then the above classes of maps form absolute and positive level model structures on $\mathcal{C}^{\mathcal{I}}$. Both level model structures are cofibrantly generated, and they are proper if each $\mathcal{C}_{\mathbf{m}}$ is.

Proof. For the absolute level model structure, we define

$$
I=\left\{F_{\mathbf{m}}(i) \mid \mathbf{m} \in \mathcal{I}, i \in I_{\mathbf{m}}\right\} \quad \text { and } \quad J=\left\{F_{\mathbf{m}}(j) \mid \mathbf{m} \in \mathcal{I}, j \in J_{\mathbf{m}}\right\},
$$

where $I_{\mathbf{m}}$ (respectively $J_{\mathbf{m}}$ ) is a set of generating (respectively generating acyclic) cofibrations for $\mathcal{C}_{\mathbf{m}}$. Now we apply the recognition theorem for cofibrantly generated model structures [25, Theorem 2.1.19]. The least obvious condition to check is that the relative $J$-cell complexes are absolute level equivalences. To see this, we use that colimits in $\mathcal{C}^{\mathcal{I}}$ are formed levelwise and deduce from (3.8) that for $j \in J_{\mathbf{m}}$ and $\mathbf{n}$ in $\mathcal{I}$, the map $F_{\mathbf{m}}(j)_{\mathbf{n}}$ is an acyclic cofibration in $\mathcal{C}_{\mathbf{m}}$ because the $\alpha$ ! are left Quillen. The fact that transfinite compositions of cobase changes 
of acyclic cofibrations in $\mathcal{C}_{\mathbf{n}}$ are weak equivalences shows that the relative $J$-cell complexes are absolute level equivalences.

To treat the positive level model structure, we write $\mathcal{I}_{\geqslant 1}$ for the full subcategory of $\mathcal{I}$ on the objects $\mathbf{m}$ with $|\mathbf{m}| \geqslant 1$, define

$$
I=\left\{F_{\mathbf{m}}(i) \mid \mathbf{m} \in \mathcal{I}_{\geqslant 1}, i \in I_{\mathbf{m}}\right\} \quad \text { and } \quad J=\left\{F_{\mathbf{m}}(j) \mid \mathbf{m} \in \mathcal{I}_{\geqslant 1}, j \in J_{\mathbf{m}}\right\}
$$

and argue as before. The statement about the relative $J$-cell complexes in the absolute case implies the one in the positive case.

EXAMPLE 3.13. We now discuss how various well-known categories of relevance for us can be expressed in terms of Quillen $\mathcal{I}$-categories $\mathcal{C}: \mathcal{I} \rightarrow$ Cat. Here $\mathcal{S}$ and $\mathcal{S}_{*}$ are equipped with the standard model structures, and $\mathcal{S}_{\mathcal{R}}$ and $\mathcal{S}_{K}$ are equipped with the model structures discussed in the previous section.

(i) Let $\mathcal{C}_{\mathbf{m}}=\mathcal{S}$ and $\alpha_{\text {! }}=$ id for all $\mathbf{m}$ and $\alpha$. Then the section category $\mathcal{C}^{\mathcal{I}}$ is the functor category $\mathcal{S}^{\mathcal{I}}$ of $\mathcal{I}$-spaces, and the model structures of Proposition 3.10 are the absolute and positive level model structure on $\mathcal{I}$ spaces that arise from [42, Proposition 6.7].

(ii) For each $\mathbf{m}$ in $\mathcal{I}$, let $\mathcal{C}_{\mathbf{m}}$ be the category of based spaces $\mathcal{S}_{*}$. The functor $\alpha_{!}: \mathcal{S}_{*} \rightarrow \mathcal{S}_{*}$ induced by $\alpha: \mathbf{m} \rightarrow \mathbf{n}$ is defined to be $-\wedge S^{\mathbf{n}-\alpha}$, the smash product with the sphere $S^{\mathbf{n}-\alpha}$ indexed by the finite set $\mathbf{n}-\alpha$. We note that the coherence isomorphism of the smash product and the isomorphisms (3.2) equip $\mathcal{C}$ with the structure of a pseudofunctor. The section category of this pseudofunctor is equivalent to the usual category of symmetric spectra $\mathrm{Sp}^{\Sigma}$. Here the structure maps in the definition of the section category correspond to the generalized structure maps of symmetric spectra (see, for example, [45, Section 3.1]). Under this equivalence of categories, the model structures of Proposition 3.10 correspond to the absolute and positive level model structures on $\mathrm{Sp}^{\Sigma}$.

(iii) Analogously to (i), the section category of the constant pseudofunctor with value $\mathcal{S}_{\mathcal{R}}$ is equivalent to $\mathcal{S}_{\mathcal{R}}^{\mathcal{I}}$, the category of $\mathcal{I}$-diagrams in retractive spaces which is in turn equivalent to the category of retractive objects in $\mathcal{I}$ spaces. Under the latter equivalence, the model structures of Proposition 3.10 correspond to the model structures on retractive objects in the absolute or positive level model structures on $\mathcal{S}^{\mathcal{I}}$ that arise by the argument in the proof of Proposition 2.5.

(iv) Let $X$ be an $\mathcal{I}$-space. We define $\mathcal{C}_{\mathbf{m}}$ to be $\mathcal{S}_{X(\mathbf{m})}$, the category of spaces over and under $X(\mathbf{m})$, and $\alpha_{!}=(X(\mathbf{m}) \rightarrow X(\mathbf{n}))_{!}: \mathcal{S}_{X(\mathbf{m})} \rightarrow \mathcal{S}_{X(\mathbf{n})}$ as 
in (2.11). The universal property of the pushout gives rise to coherence isomorphisms making this a pseudofunctor. Its section category is equivalent to $\mathcal{S}_{X}^{\mathcal{I}}$, the category of $\mathcal{I}$-spaces over and under $X$. The model structures of Proposition 3.10 correspond to those induced by the absolute and positive $\mathcal{I}$-model structure on the category of objects over and under $X$ in the usual way.

By mixing (ii) and (iii), we obtain a pseudofunctor with each $\mathcal{C}_{\mathbf{m}}$ the category of retractive spaces $\mathcal{S}_{\mathcal{R}}$ and with $\alpha_{!}: \mathcal{S}_{\mathcal{R}} \rightarrow \mathcal{S}_{\mathcal{R}}$ being the functor $-\bar{\wedge} S^{\mathbf{n}-\alpha}$ (where $S^{\mathbf{n}-\alpha}$ is a shorthand notation for $\left(S^{\mathbf{n}-\alpha}, *\right)$; see Construction 2.2).

DEFINITION 3.14. The section category of this pseudofunctor defines the category of symmetric spectra in retractive spaces $\mathrm{Sp}_{\mathcal{R}}^{\Sigma}$.

Explicitly, an object $(E, X)$ in $\operatorname{Sp}_{\mathcal{R}}^{\Sigma}$ is a sequence of retractive spaces $(E, X)_{\mathbf{m}}$ for $\mathbf{m}$ in $\mathcal{I}$ with structure maps $\alpha_{!}:(E, X)_{\mathbf{m}} \bar{\wedge}^{\mathbf{n}-\alpha} \rightarrow(E, X)_{\mathbf{n}}$ for each $\alpha: \mathbf{m} \rightarrow \mathbf{n}$ in $\mathcal{I}$ such that the obvious diagrams commute. As we will see (and heavily exploit) below, the base spaces $X(\mathbf{m})$ of $(E, X)$ assemble to an $\mathcal{I}$-space. Analogous to the discussion in (ii), one can check that the category $\mathrm{Sp}_{\mathcal{R}}^{\Sigma}$ is equivalent to the category $\operatorname{Sp}^{\Sigma}\left(\mathcal{S}_{\mathcal{R}}, S^{1}\right)$ of symmetric spectrum objects in $\mathcal{S}_{\mathcal{R}}$ with suspension functor $-\bar{\wedge} S^{1}$; see [26, Definition 7.2]. Under this equivalence, the absolute level model structure on $\mathrm{Sp}_{\mathcal{R}}^{\Sigma}$ corresponds to the level model structure established in [26, Theorem 8.2].

Let $\mathcal{C}$ and $\mathcal{D}$ be Quillen $\mathcal{I}$-categories and let $\Phi: \mathcal{C} \rightarrow \mathcal{D}$ be a pseudonatural transformation [8, Definition 7.5.2], that is, a family of functors $\Phi_{\mathbf{m}}: \mathcal{C}_{\mathbf{m}} \rightarrow \mathcal{D}_{\mathbf{m}}$ together with natural isomorphisms $\alpha_{!} \Phi_{\mathbf{m}} \stackrel{\cong}{\rightarrow} \Phi_{\mathbf{n}} \alpha_{\text {! }}$ of functors $\mathcal{C}_{\mathbf{m}} \rightarrow \mathcal{D}_{\mathbf{n}}$ that are compatible with the coherence isomorphisms of $\mathcal{C}$ and $\mathcal{D}$. Then $\Phi$ induces a functor $\Phi: \mathcal{C}^{\mathcal{I}} \rightarrow \mathcal{D}^{\mathcal{I}}$ of section categories. For $X$ in $\mathcal{C}^{\mathcal{I}}$, the object $\Phi(X)$ consists of the family of objects $\Phi_{\mathbf{m}}\left(X_{\mathbf{m}}\right)$ together with structure maps

$$
\alpha_{!}\left(\Phi_{\mathbf{m}}\left(X_{\mathbf{m}}\right)\right) \stackrel{\cong}{\rightarrow} \Phi_{\mathbf{n}}\left(\alpha_{!}\left(X_{\mathbf{m}}\right)\right) \rightarrow \Phi_{\mathbf{n}}\left(X_{\mathbf{n}}\right)
$$

induced by the structure maps of $X$ and the coherence isomorphism of $\Phi$.

LEMMA 3.15. Let $\Phi: \mathcal{C} \rightarrow \mathcal{D}$ be a pseudonatural transformation of Quillen $\mathcal{I}$ categories with each $\Phi_{\mathbf{m}}: \mathcal{C}_{\mathbf{m}} \rightarrow \mathcal{D}_{\mathbf{m}}$ a left Quillen functor. Then $\Phi: \mathcal{C}^{\mathcal{I}} \rightarrow \mathcal{D}^{\mathcal{I}}$ is a left Quillen functor with respect to the absolute and positive level model structures.

Proof. We fix right adjoints $\Psi_{\mathbf{n}}$ and units and counits for each of these adjunctions. The inverses of the coherence isomorphisms $\alpha_{!} \Phi_{\mathbf{m}} \rightarrow \Phi_{\mathbf{n}} \alpha_{!}$and the units and 
counits give rise to natural maps $\alpha_{!} \Psi_{\mathbf{m}} \rightarrow \Psi_{\mathbf{n}} \alpha_{!}$that equip the $\Psi_{\mathrm{m}}$ with the structure of a left op-lax natural transformation in the sense of [50, Definition 3.1.2]. The $\Psi_{\mathrm{m}}$ induce a functor $\Psi: \mathcal{D}^{\mathcal{I}} \rightarrow \mathcal{C}^{\mathcal{I}}$ on section categories where $\Psi(Y)$ has structure maps $\alpha_{!} \Psi_{\mathbf{m}}\left(Y_{\mathbf{m}}\right) \rightarrow \Psi_{\mathbf{n}} \alpha_{!}\left(Y_{\mathbf{m}}\right) \rightarrow \Psi_{\mathbf{n}}\left(Y_{\mathbf{n}}\right)$. Then $\Phi: \mathcal{C}^{\mathcal{I}} \rightleftarrows \mathcal{D}^{\mathcal{I}}: \Psi$ is an adjunction, and it is immediate from the definition of the fibrations and weak equivalences that $\Phi$ is right Quillen.

CONSTRUCTION 3.16. We will now apply Lemma 3.15 to various pseudonatural transformations relating the Quillen $\mathcal{I}$-categories appearing in Example 3.13(i)(iii) and in Definition 3.14. Using the functors of Construction 2.2 and Lemma 2.3, the values of the pseudonatural transformations are the horizontal arrows in the following diagrams where all squares commute up to isomorphism:

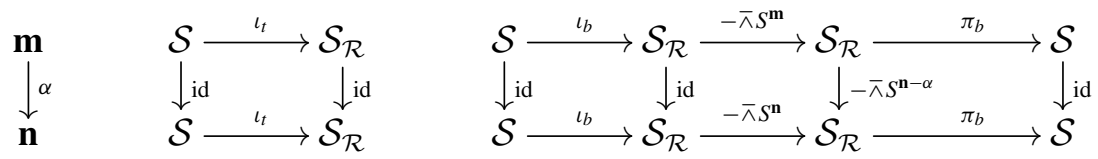

Hence, we get left Quillen functors

$$
\mathcal{S}^{\mathcal{I}} \stackrel{t_{t}}{\longrightarrow} \mathcal{S}_{\mathcal{R}}^{\mathcal{I}} \quad \mathcal{S}^{\mathcal{I}} \stackrel{\iota_{b}}{\longrightarrow} \mathcal{S}_{\mathcal{R}}^{\mathcal{I}} \stackrel{\mathbb{S}_{\mathcal{R}}^{\mathcal{I}}}{\longrightarrow} \operatorname{Sp}_{\mathcal{R}}^{\Sigma} \stackrel{\pi_{b}}{\longrightarrow} \mathcal{S}^{\mathcal{I}}
$$

Explicitly, the values of the functors $\iota_{t}$ and $\iota_{b}$ on an $\mathcal{I}$-space $X$ are

$$
\iota_{t}(X)(\mathbf{m})=(X(\mathbf{m}) \amalg X(\mathbf{m}), X(\mathbf{m})) \quad \text { and } \quad \iota_{b}(X)(\mathbf{m})=(X(\mathbf{m}), X(\mathbf{m})),
$$

and their right adjoints $\mathcal{S}_{\mathcal{R}}^{\mathcal{I}} \rightarrow \mathcal{S}^{\mathcal{I}}$ are the projections to the base and total $\mathcal{I}$ spaces. The functor $\mathbb{S}_{\mathcal{R}}^{\mathcal{I}}$ sends an object $(Z, X)$ in $\mathcal{S}_{\mathcal{R}}^{\mathcal{I}}$ to the symmetric spectrum in $\mathcal{S}_{\mathcal{R}}$ given in degree $m$ by $(Z(\mathbf{m}), X(\mathbf{m})) \bar{\wedge} S^{\mathbf{m}}$. Its right adjoint $\Omega_{\mathcal{R}}^{\mathcal{I}}$ is given by $\Omega_{\mathcal{R}}^{\mathcal{I}}(E, X)(\mathbf{m})=\operatorname{Hom}_{\mathcal{R}}\left(\left(S^{\mathbf{m}}, *\right),(E, X)(\mathbf{m})\right)$. We obtain two composite functors

$$
\mathbb{S}_{b}^{\mathcal{I}}=\mathbb{S}_{\mathcal{R}}^{\mathcal{I}} \circ \iota_{b}: \mathcal{S}^{\mathcal{I}} \rightarrow \operatorname{Sp}_{\mathcal{R}}^{\Sigma} \quad \text { and } \quad \mathbb{S}_{t}^{\mathcal{I}}=\mathbb{S}_{\mathcal{R}}^{\mathcal{I}} \circ \iota_{t}: \mathcal{S}^{\mathcal{I}} \rightarrow \operatorname{Sp}_{\mathcal{R}}^{\Sigma}
$$

Their evaluations on an $\mathcal{I}$-space $X$ are given in level $\mathbf{m}$ by

$$
\mathbb{S}_{b}^{\mathcal{I}}[X]_{m}=(X(\mathbf{m}), X(\mathbf{m})) \quad \text { and } \quad \mathbb{S}_{t}^{\mathcal{I}}[X]_{m}=\left(X(\mathbf{m}) \times S^{\mathbf{m}}, X(\mathbf{m})\right) .
$$

Their right adjoints $\pi_{b}$ and $\Omega_{t}^{\mathcal{I}}$ are given by composing $\Omega_{\mathcal{R}}^{\mathcal{I}}$ with the projection to the base and total $\mathcal{I}$-space. Finally, the functor $\pi_{b}$ in (3.17) is the projection to the underlying $\mathcal{I}$-space $X$ of a symmetric spectrum in retractive spaces $(E, X)$.

LEMMA 3.19. The functor $\mathbb{S}_{b}^{\mathcal{I}}: \mathcal{S}^{\mathcal{I}} \rightarrow \operatorname{Sp}_{\mathcal{R}}^{\Sigma}$ is both left and right adjoint to $\pi_{b}$ and both left and right Quillen with respect to the absolute and positive level model structures. 
Proof. The adjunction statement follows from Lemma 2.3. The two adjunctions $\left(\mathbb{S}_{b}^{\mathcal{I}}, \pi_{b}\right)$ and $\left(\pi_{b}, \mathbb{S}_{b}^{\mathcal{I}}\right)$ are Quillen adjunctions since $\mathbb{S}_{b}^{\mathcal{I}}=\mathbb{S}_{\mathcal{R}}^{\mathcal{I}} \circ \iota_{b}$ and $\pi_{b}$ are left Quillen.

3.20. The category of $X$-relative symmetric spectra. Let $X$ be an $\mathcal{I}$-space. By combining parts (ii) and (iv) of Example 3.13, we get another Quillen $\mathcal{I}$ category with values $\mathcal{C}_{\mathbf{m}}=\mathcal{S}_{X(\mathbf{m})}$ on objects. In this case, we define $\alpha_{!}: \mathcal{S}_{X(\mathbf{m})} \rightarrow$ $\mathcal{S}_{X(\mathbf{n})}$ to be the composite

$$
\mathcal{S}_{X(\mathbf{m})} \stackrel{X(\alpha)}{\longrightarrow} \mathcal{S}_{X(\mathbf{n})} \stackrel{-\bar{\wedge}\left(S^{\mathbf{n}-\alpha}\right)}{\longrightarrow} \mathcal{S}_{X(\mathbf{n})} .
$$

The universal property of the pushout, the coherence isomorphism of the symmetric monoidal product $\bar{\wedge}$ on $\mathcal{S}_{\mathcal{R}}$ and the isomorphisms (3.2) provide the coherence isomorphisms for this pseudofunctor.

Definition 3.22. Let $X$ be an $\mathcal{I}$-space. Then the section category of the previous Quillen $\mathcal{I}$-category is the category of $X$-relative symmetric spectra $\operatorname{Sp}_{X}^{\Sigma}$. We will also refer to it as the category of symmetric spectra parametrized by $X$.

When $K$ is a space, the category $\mathrm{Sp}_{K}^{\Sigma}=\mathrm{Sp}_{\text {const }_{\mathcal{I}} K}^{\Sigma}$ is equivalent to the category $\operatorname{Sp}^{\Sigma}\left(\mathcal{S}_{K}, S^{1}\right)$ of symmetric spectrum objects in the category $\mathcal{S}_{K}$ of spaces over and under $K$, and the absolute level model structure on $\mathrm{Sp}_{K}^{\Sigma}$ corresponds to the level model structure from [26, Theorem 8.2].

Lemma 3.23. A map of $\mathcal{I}$-spaces $f: X \rightarrow Y$ induces a Quillen adjunction

$$
f_{!}: \mathrm{Sp}_{X}^{\Sigma} \rightleftarrows \operatorname{Sp}_{Y}^{\Sigma}: f^{*}
$$

with respect to the absolute and positive model structures.

Proof. We apply Lemma 3.15 to the functors $f(\mathbf{m})_{!}: \mathcal{S}_{X(\mathbf{m})} \rightarrow \mathcal{S}_{Y(\mathbf{m})}$.

Analogous to Lemma 2.12, we obtain a pseudofunctor

$$
X \mapsto \mathrm{Sp}_{X}^{\Sigma}, \quad(f: X \rightarrow Y) \mapsto\left(f_{!}: \mathrm{Sp}_{X}^{\Sigma} \rightarrow \mathrm{Sp}_{Y}^{\Sigma}\right)
$$

LEMMA 3.26. The Grothendieck construction of (3.25) is equivalent to $\mathrm{Sp}_{\mathcal{R}}^{\Sigma}$.

Proof. This follows from a levelwise application of Lemma 2.14.

Under this equivalence, the category $\operatorname{Sp}_{X}^{\Sigma}$ corresponds to the fiber of $\pi_{b}$ over $X$. This identification of $\operatorname{Sp}_{X}^{\Sigma}$ allows us to give a different description of the 
adjunction (3.24): there are natural isomorphisms

$$
f_{!}(E, X) \cong(E, X) \cup_{\mathbb{S}_{b}^{\mathcal{I}}[X]} \mathbb{S}_{b}^{\mathcal{I}}[Y] \quad \text { and } \quad f^{*}(F, Y) \cong(F, Y) \times_{\mathbb{S}_{b}^{\mathcal{I}}[Y]} \mathbb{S}_{b}^{\mathcal{I}}[X]
$$

where the pushouts and pullbacks are formed in $\mathrm{Sp}_{\mathcal{R}}^{\Sigma}$. In other words, $f_{!}$ corresponds to the cobase change along $\mathbb{S}_{b}^{\mathcal{I}}[f]$, and $f^{*}$ corresponds to the base change along $\mathbb{S}_{b}^{\mathcal{I}}[f]$.

We write $F_{\mathbf{m}}^{\mathrm{Sp}_{\mathcal{R}}^{\Sigma}}: \mathcal{S}_{\mathcal{R}} \rightarrow \mathrm{Sp}_{\mathcal{R}}^{\Sigma}, F_{\mathbf{m}}^{\mathrm{Sp}_{X}^{\Sigma}}: \mathcal{S}_{X(\mathbf{m})} \rightarrow \mathrm{Sp}_{X}^{\Sigma}$, and $F_{\mathbf{m}}^{\mathcal{S}^{\mathcal{I}}}: \mathcal{S} \rightarrow \mathcal{S}^{\mathcal{I}}$ for the free functors obtained by implementing (3.7) in the categories $\operatorname{Sp}_{\mathcal{R}}^{\Sigma}, \operatorname{Sp}_{X}^{\Sigma}$, and $\mathcal{S}^{\mathcal{I}}$.

For later use, we record how the free functors to $\mathrm{Sp}_{\mathcal{R}}^{\Sigma}$ and $\mathrm{Sp}_{X}^{\Sigma}$ are related.

LEMMA 3.28. Let $(V, L)$ be an object in $\mathcal{S}_{L}$, let $f: F_{\mathbf{m}}^{\mathcal{S}^{\mathcal{I}}}(L) \rightarrow X$ be a map in $\mathcal{S}^{\mathcal{I}}$, and let $\widetilde{f}: L \rightarrow X(\mathbf{m})$ be the adjoint of $f$. Then there is a natural isomorphism $F_{\mathbf{m}}^{\mathrm{Sp}_{X}^{\Sigma}}\left(\tilde{f}_{!}(V, L)\right) \cong f_{!} F_{\mathbf{m}}^{\mathrm{Sp}_{\mathcal{R}_{\mathcal{R}}}^{\Sigma}}(V, L)$.

Applying Definition 2.17 to the pseudofunctor (3.25), the absolute and positive level model structures on $\mathcal{S}^{\mathcal{I}}$ and the $\mathrm{Sp}_{X}^{\Sigma}$ give rise to integral cofibrations, fibrations, and weak equivalences on the Grothendieck construction.

PROPOSITION 3.29. These classes of maps form absolute and positive integral level model structures on the Grothendieck construction. Under the equivalence with $\mathrm{Sp}_{\mathcal{R}}^{\Sigma}$, they correspond to the absolute and positive level model structures on $\mathrm{Sp}_{\mathcal{R}}^{\Sigma}$.

Proof. Inspecting the generating cofibrations of $\mathcal{S}^{\mathcal{I}}$, we see that a cofibration in the absolute level model structure on $\mathcal{S}^{\mathcal{I}}$ is levelwise a cofibration in $\mathcal{S}$. A similar result holds for the absolute level model structure on $\operatorname{Sp}_{X}^{\Sigma}$. It follows that the analogue of Lemma 2.16 holds for the adjunction (3.24). Hence, [20, Theorem 3.0.12] applies and shows the existence of the integral model structure. It matches with the absolute level model structure on $\mathrm{Sp}_{\mathcal{R}}^{\Sigma}$ since the fibrations and weak equivalences are the same. The case of the positive model structures is analogous.

This proposition, the definition of the integral model structure, and the identification of $\operatorname{Sp}_{X}^{\Sigma}$ with the fiber of $\pi_{b}: \mathrm{Sp}_{\mathcal{R}}^{\Sigma} \rightarrow \mathcal{S}^{\mathcal{I}}$ over $X$ imply the following.

COROLlary 3.30. A map in the absolute (respectively positive) level model structure on $\mathrm{Sp}_{X}^{\Sigma}$ is a cofibration, fibration, or weak equivalence if and only if it is so when viewed as a map in the absolute (respectively positive) model structure on $\mathrm{Sp}_{\mathcal{R}}^{\Sigma}$. 


\section{The convolution smash product}

The reason for using $\mathcal{I}$ as an indexing category in the definition of a Quillen $\mathcal{I}$ category is that this allows us to define symmetric monoidal products on section categories.

In the case of $\mathcal{I}$-spaces mentioned in Example 3.13(i), the monoidal product $X \otimes Y$ of two functors $X, Y: \mathcal{I} \rightarrow \mathcal{S}$ is the left Kan extension of the $\mathcal{I} \times \mathcal{I}$ diagram $(\mathbf{m}, \mathbf{n}) \mapsto X(\mathbf{m}) \times Y(\mathbf{n})$ along the concatenation $-\sqcup-: \mathcal{I} \times \mathcal{I} \rightarrow \mathcal{I}$. This is an example of a Day convolution product, and more explicitly, we have

$$
(X \otimes Y)(\mathbf{p})=\operatorname{colim}_{\mathbf{m} \sqcup \mathbf{n} \rightarrow \mathbf{p}} X(\mathbf{m}) \times Y(\mathbf{n})
$$

with the colimit taken over the over-category $-\sqcup-\downarrow \mathbf{p}$. The $\bigotimes$-product provides a symmetric monoidal product on $\mathcal{S}^{\mathcal{I}}$ with unit $*=F_{\mathbf{0}}^{\mathcal{S}^{\mathcal{I}}}(*) \cong \mathcal{I}(\mathbf{0},-) \cong$ $\operatorname{const}_{\mathcal{I}}(*)$.

DEFINITION 4.1. A commutative $\mathcal{I}$-space monoid is a commutative monoid in $\left(\mathcal{S}^{\mathcal{I}}, \bigotimes, *\right)$.

Equivalently, a commutative $\mathcal{I}$-space monoid is a lax symmetric monoidal functor $(\mathcal{I}, \sqcup, \mathbf{0}) \rightarrow\left(\mathcal{S}^{\mathcal{I}}, \bigotimes, *\right)$. Every $E_{\infty}$ space can be represented by a commutative $\mathcal{I}$-space monoid in the sense explained in [42, Corollary 3.7].

In the case of symmetric spectra mentioned in Example 3.13(ii), the monoidal product is the well-known smash product of symmetric spectra. In this description of symmetric spectra employing $\mathcal{I}$, the smash product $E \wedge F$ of $E, F$ is in level $p$ given by the colimit

$$
(E \wedge F)_{p}=\operatorname{colim}_{\alpha: \mathbf{m} \sqcup \mathbf{n} \rightarrow \mathbf{p}} E_{m} \wedge F_{n} \wedge S^{\mathbf{p}-\alpha}
$$

taken over the over-category $-\sqcup-\downarrow \mathbf{p}$. The maps in the colimit system arise from the structure maps of $E$ and $F$, the isomorphism (3.1), and the isomorphism

$$
S^{\left(\mathbf{n}_{1} \sqcup \mathbf{n}_{2}\right)-\left(\alpha_{1} \sqcup \alpha_{2}\right)} \cong S^{\mathbf{n}_{1}-\alpha_{1}} \wedge S^{\mathbf{n}_{2}-\alpha_{2}}
$$

that is induced by the canonical bijection

$$
\left(\mathbf{n}_{1}-\alpha_{1}\right) \amalg\left(\mathbf{n}_{2}-\alpha_{2}\right) \rightarrow\left(\mathbf{n}_{1} \sqcup \mathbf{n}_{2}\right)-\left(\alpha_{1} \sqcup \alpha_{2}\right)
$$

associated with a pair of morphisms $\alpha_{1}: \mathbf{m}_{1} \rightarrow \mathbf{n}_{\mathbf{1}}$ and $\alpha_{2}: \mathbf{m}_{2} \rightarrow \mathbf{n}_{2}$ in $\mathcal{I}$. The structure maps of $E \wedge F$ also arise from (3.1). 
4.3. The monoidal structure on symmetric spectra in retractive spaces. In analogy with the smash product in $\mathrm{Sp}^{\Sigma}$ and the $\otimes$-product of $\mathcal{I}$-spaces, there is a symmetric monoidal product

$$
-\bar{\wedge}-: \mathrm{Sp}_{\mathcal{R}}^{\Sigma} \times \mathrm{Sp}_{\mathcal{R}}^{\Sigma} \rightarrow \mathrm{Sp}_{\mathcal{R}}^{\Sigma}
$$

given in level $p$ by the colimit

$$
((E, X) \bar{\wedge}(F, Y))_{p}=\operatorname{colim}_{\alpha: \mathbf{m} \sqcup \mathbf{n} \rightarrow \mathbf{p}}\left(E_{m}, X_{m}\right) \bar{\wedge}\left(F_{n}, X_{n}\right) \bar{\wedge} S^{\mathbf{p}-\alpha}
$$

in $\mathcal{S}_{\mathcal{R}}$ taken over the category $-\sqcup-\downarrow \mathbf{p}$. The maps in the colimit system and the structure maps of $(E, X) \bar{\wedge}(F, Y)$ are defined as for symmetric spectra. We also note that there are natural isomorphisms

$$
F_{\mathbf{m}}^{\mathrm{Sp}_{\mathcal{R}}^{\Sigma}}(U, K) \bar{\wedge} F_{\mathbf{n}}^{\mathrm{Sp}_{\mathcal{R}}^{\Sigma}}(V, L) \cong F_{\mathbf{m} \sqcup \mathbf{n}}^{\mathrm{Sp}_{\mathcal{R}}^{\Sigma}}((U, K) \bar{\wedge}(V, L))
$$

PROPOSITION 4.5. The $\bar{\wedge}$-product defines a closed symmetric monoidal structure on $\mathrm{Sp}_{\mathcal{R}}^{\Sigma}$ with unit $\mathbb{S}$ that satisfies the pushout product axiom with respect to the absolute and positive level model structures.

Proof. Since the pushout product axiom can be checked on the generating (acyclic) cofibrations, it follows from the isomorphisms (4.4) and the pushout product axiom for $\mathcal{S}_{\mathcal{R}}$ established in Proposition 2.27. The $\bar{\wedge}$-product on $\operatorname{Sp}_{\mathcal{R}}^{\Sigma}$ is closed because $\left(\mathcal{S}_{\mathcal{R}}, \bar{\wedge}, S^{0}\right)$ is closed by Proposition 2.24 (compare [26, Section 7]).

We write $E \bar{\wedge} F$ for the total space of $(E, X) \bar{\wedge}(F, Y)$. Observing that its base $\mathcal{I}$-space can be identified with $X \otimes Y$, we have

$$
(E, X) \bar{\wedge}(F, Y)=(E \bar{\wedge} F, X \otimes Y)
$$

LEMMA 4.6. If $(E, X)$ and $(F, Y)$ are objects in $\mathrm{Sp}_{\mathcal{R}}^{\Sigma}$ and $f: X \rightarrow X^{\prime}$ and $g: Y \rightarrow Y^{\prime}$ are morphisms in $\mathcal{S}^{\mathcal{I}}$, then there is an isomorphism

$$
f_{!}(E, X) \bar{\wedge} g_{!}(F, Y) \stackrel{\cong}{\rightarrow}(f \otimes g)_{!}((E, X) \bar{\wedge}(F, Y)) .
$$

It is natural with respect to the coherence isomorphisms $\left(f^{\prime} f\right)_{!} \cong f_{!}^{\prime} f_{!}$for composable maps of $\mathcal{I}$-spaces $f$ and $f^{\prime}$.

Proof. Commuting the colimit over $-\sqcup-\downarrow \mathbf{p}$ with the pushout computing the total space identifies the total space in level $p$ of the right-hand expression with 
$\operatorname{colim}_{\alpha: \mathbf{m} \sqcup \mathbf{n} \rightarrow \mathbf{p}}(f(\mathbf{m}) \times g(\mathbf{n}))_{!}\left(E_{m} \bar{\wedge} F_{m} \bar{\wedge} S^{\mathbf{p}-\alpha}\right)$. Composing it with the colimit over $\alpha$ in $-\sqcup-\downarrow \mathbf{p}$ of the natural isomorphisms

$$
\begin{aligned}
& f(\mathbf{m})_{!}\left(E_{m}, X(\mathbf{m})\right) \bar{\wedge} g(\mathbf{n})_{!}\left(F_{n}, Y(\mathbf{n})\right) \bar{\wedge} S^{\mathbf{p}-\alpha} \\
& \quad \stackrel{\cong}{\rightarrow}(f(\mathbf{m}) \times g(\mathbf{n}))_{!}\left(\left(E_{m}, X(\mathbf{m})\right) \bar{\wedge}\left(F_{n}, F(\mathbf{n})\right) \bar{\wedge} S^{\mathbf{p}-\alpha}\right)
\end{aligned}
$$

provided by Lemma 2.28 gives the desired isomorphism.

As a consequence, we note that given maps of $\mathcal{I}$-spaces $f: X^{\prime} \rightarrow X$ and $g: Y^{\prime} \rightarrow Y$ as well as objects $(E, X)$ in $\operatorname{Sp}_{X}^{\Sigma}$ and $(F, Y)$ in $\operatorname{Sp}_{Y}^{\Sigma}$, there is a chain of maps

$$
\begin{aligned}
& f^{*}(E, X) \bar{\wedge} g^{*}(F, Y) \rightarrow(f \otimes g)^{*}(f \otimes g) !\left(f^{*}(E, X) \bar{\wedge} g^{*}(F, Y)\right) \\
& \quad \cong(f \otimes g)^{*}\left(f_{!} f^{*}(E, X) \bar{\wedge} g_{!} g^{*}(F, Y)\right) \rightarrow(f \nabla g)^{*}((E, X) \bar{\wedge}(F, Y))
\end{aligned}
$$

induced by the adjunction unit of $\left((f \otimes g)_{!},(f \otimes g)^{*}\right)$, the isomorphism of Lemma 4.6, and the adjunction counits of $\left(f_{!}, f^{*}\right)$ and $\left(g_{!}, g^{*}\right)$. We will show in Proposition 7.3 that this morphism descends to an isomorphism between the derived functors in the homotopy category.

The category $\mathcal{S}_{\mathcal{R}}^{\mathcal{I}}$ of $\mathcal{I}$-diagrams in $\mathcal{S}_{\mathcal{R}}$ has a Day convolution product induced by the $\bar{\wedge}$-product on $\mathcal{S}_{\mathcal{R}}$ and the concatenation in $\mathcal{I}$. Analogously, the cartesian product on $\operatorname{Ar}(\mathcal{S})$ induces a Day convolution product on $(\operatorname{Ar}(\mathcal{S}))^{\mathcal{I}}$ that coincides with the objectwise $\triangle$-product in $\mathcal{S}^{\mathcal{I}}$ under the identification $(\operatorname{Ar}(\mathcal{S}))^{\mathcal{I}} \cong \operatorname{Ar}\left(\mathcal{S}^{\mathcal{I}}\right)$. In the next diagram, the first adjunction is induced by the corresponding space level adjunction from Construction 2.2 and the second is from Construction 3.16.

$$
\operatorname{Ar}\left(\mathcal{S}^{\mathcal{I}}\right) \underset{\pi_{\mathrm{ar}}}{\stackrel{\iota_{\mathrm{ar}}}{\rightleftarrows}} \mathcal{S}_{\mathcal{R}}^{\mathcal{I}} \underset{\Omega_{\text {R }}^{\mathcal{I}}}{\stackrel{\mathbb{S}_{\mathcal{R}}^{\mathcal{I}}}{\rightleftarrows}} \operatorname{Sp}_{\mathcal{R}}^{\Sigma}
$$

LEMMA 4.9. The left adjoint functors $\iota_{\mathrm{ar}}$ and $\mathbb{S}_{\mathcal{R}}^{\mathcal{I}}$ in (4.8) are strong symmetric monoidal; hence, so is their composite $\mathbb{S}_{\mathrm{ar}}^{\mathcal{I}}=\mathbb{S}_{\mathcal{R}}^{\mathcal{I}} \circ \iota_{\mathrm{ar}}$, and the right adjoints $\pi_{\mathrm{ar}}$, $\Omega_{\mathcal{R}}^{\mathcal{I}}$, and $\Omega_{\mathrm{ar}}^{\mathcal{I}}=\pi_{\mathrm{ar}} \circ \Omega_{\mathcal{R}}^{\mathcal{I}}$ are lax symmetric monoidal.

4.10. The monoidal structure on $M$-relative symmetric spectra. Let $X$ and $Y$ be $\mathcal{I}$-spaces. Via the identification of $\mathrm{Sp}_{X}^{\Sigma}, \mathrm{Sp}_{Y}^{\Sigma}$, and $\mathrm{Sp}_{X \bowtie Y}^{\Sigma}$ with subcategories of $\mathrm{Sp}_{\mathcal{R}}^{\Sigma}$, the $\bar{\wedge}$-product on $\mathrm{Sp}_{\mathcal{R}}^{\Sigma}$ induces an external product

$$
-\bar{\wedge}-: \operatorname{Sp}_{X}^{\Sigma} \times \operatorname{Sp}_{Y}^{\Sigma} \rightarrow \operatorname{Sp}_{X \bowtie Y}^{\Sigma}
$$


If $M$ is a commutative $\mathcal{I}$-space monoid, then this external product and the multiplication $\mu: M \otimes M \rightarrow M$ of $M$ induce a symmetric monoidal convolution product

$$
\mathrm{Sp}_{M}^{\Sigma} \times \mathrm{Sp}_{M}^{\Sigma} \rightarrow \mathrm{Sp}_{M \otimes M}^{\Sigma} \stackrel{\mu_{1}}{\rightarrow} \mathrm{Sp}_{M}^{\Sigma}
$$

Let $\iota: * \rightarrow M$ be the unit and write $\mathbb{S}_{M}=\iota_{!}(\mathbb{S})$, where $\mathbb{S}=(\mathbb{S}, *)$ is the monoidal unit in $\mathrm{Sp}^{\Sigma}=\mathrm{Sp}_{*}^{\Sigma}$. It follows from Lemma 4.6 that $\mathbb{S}_{M}$ is the monoidal unit for $\mathrm{Sp}_{M}^{\Sigma}$.

Proposition 4.13. This symmetric monoidal product satisfies the pushout product axiom with respect to the absolute level model structure on $\mathrm{Sp}_{M}^{\Sigma}$.

Proof. This follows from the pushout product axiom for $\mathrm{Sp}_{\mathcal{R}}^{\Sigma}$ and the fact that $\mu$ ! preserves cofibrations and acyclic cofibrations by Lemma 3.23.

In a similar fashion, the category $\mathcal{S}_{M}^{\mathcal{I}}$ of $\mathcal{I}$-spaces over and under $M$ inherits a symmetric monoidal product from $\mathcal{S}_{\mathcal{R}}^{\mathcal{I}}$. For later use, we note the following compatibility.

LEMMA 4.14. The functor $\mathbb{S}_{\mathcal{R}}^{\mathcal{I}}: \mathcal{S}_{M}^{\mathcal{I}} \rightarrow \mathrm{Sp}_{M}^{\Sigma}$ is strong symmetric monoidal.

If $M \rightarrow N$ is a morphism of commutative $\mathcal{I}$-space monoids, then Lemma 4.6 implies that the induced functor $(M \rightarrow N)_{!}: \operatorname{Sp}_{M}^{\Sigma} \rightarrow \operatorname{Sp}_{N}^{\Sigma}$ is strong symmetric monoidal. In particular,

$$
\Theta=(M \rightarrow *) !: \mathrm{Sp}_{M}^{\Sigma} \rightarrow \mathrm{Sp}^{\Sigma}
$$

is strong symmetric monoidal so that commutative monoids in $\operatorname{Sp}_{M}^{\Sigma}$ give rise to commutative symmetric ring spectra if their base $\mathcal{I}$-space is collapsed.

4.15. The simplicial structure on $X$-relative symmetric spectra. Let $X$ be an $\mathcal{I}$-space. If $Q$ is an unbased simplicial set, we define a functor

$$
Q \otimes-: \mathrm{Sp}_{X}^{\Sigma} \rightarrow \mathrm{Sp}_{X}^{\Sigma}, \quad(E, X) \mapsto F_{0}^{\mathrm{Sp}_{\mathcal{R}}^{\Sigma}}\left(Q_{+}\right) \bar{\wedge}(E, X)
$$

Here we again identify $\operatorname{Sp}_{X}^{\Sigma}$ with a subcategory of $\operatorname{Sp}_{\mathcal{R}}^{\Sigma}$, view $Q_{+}$as the retractive space $\left(Q_{+}, *\right)$, and apply geometric realization to $Q$ when working with $\mathcal{S}=$ Top.

PROPOSITION 4.16. This action turns $\mathrm{Sp}_{X}^{\Sigma}$ into a simplicial model category.

Proof. An application of [17, Lemma II.2.4] shows that $\mathrm{Sp}_{X}^{\Sigma}$ becomes a simplicial category since $\bar{\wedge}$ is a closed symmetric monoidal structure on $\operatorname{Sp}_{\mathcal{R}}^{\Sigma}$. The 
compatibility with the model structure follows from the pushout product axiom for $\left(\mathrm{Sp}_{\mathcal{R}}^{\Sigma}, \bar{\wedge}\right)$ established in Proposition 4.5 and the compatibility of the model structures on $\mathrm{Sp}_{X}^{\Sigma}$ and $\mathrm{Sp}_{\mathcal{R}}^{\Sigma}$ established in Corollary 3.30.

It follows from the definitions that $(Q \otimes(E, X))_{n}$ can be identified with the tensor $Q \otimes\left(E_{n}, X_{n}\right)$ in $\mathcal{S}_{X_{n}}$ (see Proposition 2.33). Since the category $\mathrm{Sp}_{X}^{\Sigma}$ has a zero object, the tensor structure over sSet induces a tensor over $\mathrm{sSet}_{*}$, and one can check that for a based simplicial set $B$, the based tensor is just the levelwise $\bar{\wedge}$-product with $(B, *)$. Particularly, the suspension is the levelwise $\bar{\wedge}$-product with $S^{1}$.

4.17. Tensor structures over $\mathcal{I}$-spaces. There is a functor

$$
\mathcal{S}^{\mathcal{I}} \times \operatorname{Sp}_{\mathcal{R}}^{\Sigma} \rightarrow \operatorname{Sp}_{\mathcal{R}}^{\Sigma}, \quad(Y,(E, X)) \mapsto \mathbb{S}_{t}^{\mathcal{I}}[Y] \bar{\wedge}(E, X)
$$

that exhibits $\operatorname{Sp}_{\mathcal{R}}^{\Sigma}$ as a category tensored over $\left(\mathcal{S}^{\mathcal{I}}, \bigotimes, *\right)$, meaning that $\operatorname{Sp}_{\mathcal{R}}^{\Sigma}$ is a $\mathcal{S}^{\mathcal{I}}$-module in the sense of [25, Definition 4.1.6]. For the applications in [21] and in Section 9.15, it is important that $\operatorname{Sp}_{\mathcal{R}}^{\Sigma}$ is also tensored over $\left(\mathcal{S}^{\mathcal{I}}, \times, *\right)$, that is, $\mathcal{I}$-spaces with the cartesian product. To define this tensor, we first introduce a monoidal product on the category $\mathcal{S}_{\mathcal{R}}^{\mathcal{I}}$ of $\mathcal{I}$-diagrams in $\mathcal{S}_{\mathcal{R}}$ and an accompanying tensor structure on $\operatorname{Sp}_{\mathcal{R}}^{\Sigma}$. The monoidal structure on $\mathcal{S}_{\mathcal{R}}^{\mathcal{I}}$ is the degreewise $\bar{\wedge}$ product and will be denoted by $\widetilde{\wedge}$. Its unit is $\iota_{t}(*)=\operatorname{const}_{\mathcal{I}}\left(S^{0}, *\right)$, and the functor $\iota_{t}:\left(\mathcal{S}^{\mathcal{I}}, \times, *\right) \rightarrow\left(\mathcal{S}_{\mathcal{R}}^{\mathcal{I}}, \widetilde{\wedge}, \iota_{t}(*)\right)$ sending $X$ to $(X \amalg X, X)$ is strong symmetric monoidal by (2.28).

The category $\operatorname{Sp}_{\mathcal{R}}^{\Sigma}$ is tensored over $\left(\mathcal{S}_{\mathcal{R}}^{\mathcal{I}}, \widetilde{\wedge}, \iota_{t}(*)\right)$ with tensor structure

$$
-\widetilde{\wedge}-: \mathcal{S}_{\mathcal{R}}^{\mathcal{I}} \times \operatorname{Sp}_{\mathcal{R}}^{\Sigma} \rightarrow \operatorname{Sp}_{\mathcal{R}}^{\Sigma}, \quad((Z, Y),(E, X)) \mapsto(\mathbf{n} \mapsto(Z, Y)(\mathbf{n}) \bar{\wedge}(E, X)(\mathbf{n})) .
$$

Here the structure maps act diagonally, that is, $\alpha: \mathbf{m} \rightarrow \mathbf{n}$ acts via

$$
(Z, Y)(\mathbf{m}) \bar{\wedge}(E, X)(\mathbf{m}) \bar{\wedge} S^{\mathbf{n}-\alpha} \stackrel{\alpha_{*} \bar{\wedge} \alpha_{*}}{\longrightarrow}(Z, Y)(\mathbf{n}) \bar{\wedge}(E, X)(\mathbf{n}) .
$$

Restricting (4.19) along $\iota_{t}$ in the first variable, we get the desired tensor structure of $\operatorname{Sp}_{\mathcal{R}}^{\Sigma}$ over $\left(\mathcal{S}^{\mathcal{I}}, \times, *\right)$ given by

$$
-\times-: \mathcal{S}^{\mathcal{I}} \times \operatorname{Sp}_{\mathcal{R}}^{\Sigma} \rightarrow \operatorname{Sp}_{\mathcal{R}}^{\Sigma}
$$

with $(Y \times(E, X))(\mathbf{n}) \cong\left(Y(\mathbf{n}) \times E_{n}, Y(\mathbf{n}) \times X(\mathbf{n})\right)$. The latter isomorphism results from (2.27) and justifies the symbol $\times$. Although $\times$ admits this easier description, we have chosen to define it via $\widetilde{\wedge}$ since this makes the structure maps of the object $Y \times(E, X)$ more transparent. We shall primarily use the action $\times$ when $(E, X)$ is just a symmetric spectrum $E$ viewed as the object $E=(E, *)$ of $\mathrm{Sp}_{\mathcal{R}}^{\Sigma}$.

Now we relate this tensor structure to that of (4.18). 
CONSTRUCTION 4.21. There is a natural transformation

$$
\rho_{Y,(E, X)}: \mathbb{S}_{t}^{\mathcal{I}}[Y] \bar{\wedge}(E, X) \rightarrow Y \times(E, X)
$$

of functors $\mathcal{S}^{\mathcal{I}} \times \mathrm{Sp}_{\mathcal{R}}^{\Sigma} \rightarrow \mathrm{Sp}_{\mathcal{R}}^{\Sigma}$. On the term in the colimit system defining the $\bar{\wedge}$-product that is indexed by $\alpha: \mathbf{k} \sqcup \mathbf{I} \rightarrow \mathbf{n}$, it is given by the composite

$$
\begin{aligned}
\left(\iota_{t} Y\right)(\mathbf{k}) \bar{\wedge} S^{\mathbf{k}} \bar{\wedge}(E, X)(\mathbf{l}) \bar{\wedge} S^{\mathbf{n}-\alpha} & \cong\left(\iota_{t} Y\right)(\mathbf{k}) \bar{\wedge}(E, X)(\mathbf{l}) \bar{\wedge} S^{\mathbf{n}-\left.\alpha\right|_{\mathbf{1}}} \\
& \rightarrow\left(\iota_{t} Y\right)(\mathbf{n}) \bar{\wedge}(E, X)(\mathbf{n})=(Y \times(E, X))(\mathbf{n}) .
\end{aligned}
$$

Here the first map interchanges the two inner factors and uses the isomorphism of spheres induced by the bijection $\mathbf{k} \sqcup(\mathbf{n}-\alpha) \rightarrow\left(\mathbf{n}-\left.\alpha\right|_{\mathbf{l}}\right)$ defined by $\alpha$, and the second map is given by the action of $\left.\alpha\right|_{\mathbf{k}}$ and the structure map of $(E, X)$.

It is shown in [21, Proposition 4.1] that under suitable conditions on $Y$ and ( $E, X)$, the map (4.22) is a local equivalence in the sense of Section 5.7. The latter result plays a central role in our applications to models of twisted $K$-theory spectra in [21]. We also note that on base $\mathcal{I}$-spaces, $\rho_{Y,(E, X)}$ is just the natural map $Y \otimes X \rightarrow Y \times X$ studied in [43, Proposition 2.27].

It will also be useful to know that the different products are related by the following commutative square:

$$
\begin{aligned}
& \mathbb{S}_{t}^{\mathcal{I}}[Y] \bar{\wedge}(E, X) \bar{\wedge} \mathbb{S}_{t}^{\mathcal{I}}\left[Y^{\prime}\right] \bar{\wedge}\left(E^{\prime}, X^{\prime}\right) \longrightarrow \mathbb{S}_{t}^{\mathcal{I}}\left[Y \otimes Y^{\prime}\right] \bar{\wedge}(E, X) \bar{\wedge}\left(E^{\prime}, X^{\prime}\right)
\end{aligned}
$$

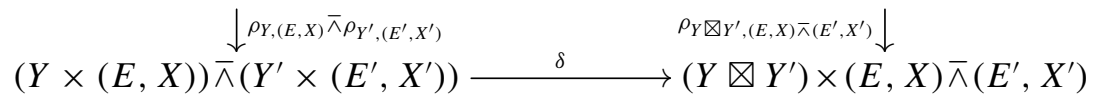

The vertical maps are instances of (4.22), the upper horizontal map is the composite of the twists of the middle terms and the isomorphism $\mathbb{S}_{t}^{\mathcal{I}}[Y] \bar{\wedge} \mathbb{S}_{t}^{\mathcal{I}}\left[Y^{\prime}\right] \rightarrow \mathbb{S}_{t}^{\mathcal{I}}\left[Y \otimes Y^{\prime}\right]$, and the lower horizontal map $\delta$ is the distributivity map induced by the maps

$$
\begin{aligned}
& (Y(\mathbf{k}) \times(E, X)(\mathbf{k})) \bar{\wedge}\left(Y^{\prime}(\mathbf{l}) \times\left(E^{\prime}, X^{\prime}\right)(\mathbf{l})\right) \bar{\wedge} S^{\mathbf{n}-\alpha} \\
& \quad \rightarrow\left(Y \otimes Y^{\prime}\right)(\mathbf{n}) \times\left((E, X) \bar{\wedge}\left(E^{\prime}, X^{\prime}\right)\right)(\mathbf{n})
\end{aligned}
$$

for $\alpha: \mathbf{k} \sqcup \mathbf{l} \rightarrow \mathbf{n}$ in $\mathcal{I}$ which are given by the twist of the middle factors and the canonical maps to the $\otimes$ and $\bar{\wedge}$-products.

\section{Local model structures}

Let $\mathcal{C}: \mathcal{I} \rightarrow$ Cat be a Quillen $\mathcal{I}$-category. If $\alpha: \mathbf{m} \rightarrow \mathbf{n}$ is a map in $\mathcal{I}$ and $Z$ is an object in $\mathcal{C}_{\mathbf{m}}$, then the inclusion $\alpha_{!} Z \rightarrow \bigsqcup_{\beta: \mathbf{m} \rightarrow \mathbf{n}} \beta_{!} Z=\left(F_{\mathbf{m}}(Z)\right)_{\mathbf{n}}$ of the 
summand indexed by $\alpha$ gives rise to an adjoint map

$$
\alpha_{Z}: F_{\mathbf{n}}(\alpha ! Z) \rightarrow F_{\mathbf{m}}(Z)
$$

in the section category $\mathcal{C}^{\mathcal{I}}$. We define $S_{\mathcal{C}}$ to be the set of maps $\alpha_{Z}$ where $\alpha: \mathbf{m} \rightarrow \mathbf{n}$ is any morphism in $\mathcal{I}$ and $Z$ is the cofibrant replacement of a domain or codomain of a generating cofibration of $\mathcal{C}_{\mathbf{m}}$. Writing $\mathcal{I}^{+}$for the full subcategory of $\mathcal{I}$ on the objects $\mathbf{m}$ with $|\mathbf{m}| \geqslant 1$, we let $S_{\mathcal{C}}^{+}$be the subset of $S_{\mathcal{C}}$ where $\alpha$ runs through the morphisms in $\mathcal{I}^{+}$.

Our aim is to form the left Bousfield localizations [23, Section 3] of the level model structures on $\mathcal{C}^{\mathcal{I}}$ at $S_{\mathcal{C}}$ and $S_{\mathcal{C}}^{+}$. We need an additional hypothesis to ensure their existence and say that a Quillen $\mathcal{I}$-category $\mathcal{C}$ is cellular and left proper if each $\mathcal{C}_{\mathbf{m}}$ is.

Proposition 5.2. Let $\mathcal{C}: \mathcal{I} \rightarrow$ Cat be a cellular and left proper Quillen $\mathcal{I}$ category. Then the left Bousfield localizations of the absolute level model structure on $\mathcal{C}^{\mathcal{I}}$ at the set $S_{\mathcal{C}}$ and the positive level model structure at $S_{\mathcal{C}}^{+}$exist and are cellular and left proper again.

Proof. This follows from [23, Theorem 4.1] once we verified that the absolute level model structure on $\mathcal{C}^{\mathcal{I}}$ is cellular and left proper. Since cofibrations in $\mathcal{C}^{\mathcal{I}}$ are in particular cofibrations in each level and colimits in $\mathcal{C}^{\mathcal{I}}$ are formed levelwise, this is immediate.

DEFINITION 5.3. The model structures from the previous proposition are called the absolute and positive local model structures on the section category $\mathcal{C}^{\mathcal{I}}$.

LEMMA 5.4. An object $X$ in $\mathcal{C}^{\mathcal{I}}$ is fibrant in the absolute (respectively positive) local model structure if and only if for each $\alpha$ in $\mathcal{I}$ (respectively $\mathcal{I}^{+}$), the adjoint structure map $X_{\mathbf{m}} \rightarrow \alpha^{*}\left(X_{\mathbf{n}}\right)$ is a weak equivalence between fibrant objects in $\mathcal{C}_{\mathbf{m}}$.

Proof. We write $\operatorname{Map}_{\mathcal{C}^{\mathcal{I}}}$ and $\operatorname{Map}_{\mathcal{C}_{\mathrm{m}}}$ for the homotopy function complexes in $\mathcal{C}^{\mathcal{I}}$ and in $\mathcal{C}_{\mathrm{m}}$ (see [23, Section 17.4]). By definition, an object $X$ is fibrant in the absolute local model structure on $\mathcal{C}^{\mathcal{I}}$ if and only if it is absolute level fibrant and $\operatorname{Map}_{\mathcal{C}^{\mathcal{I}}}\left(\alpha_{Z}, X\right)$ is a weak equivalence of simplicial sets for all $\alpha_{Z}$ in $S_{\mathcal{C}}$. Since homotopy function complexes are compatible with Quillen adjunctions [23, Proposition 17.4.15], the latter condition is equivalent to asking that the map $\operatorname{Map}_{\mathcal{C}_{\mathbf{m}}}\left(Z, X_{\mathbf{m}}\right) \rightarrow \operatorname{Map}_{\mathcal{C}_{\mathbf{m}}}\left(Z, \alpha^{*}\left(X_{\mathbf{n}}\right)\right)$ is a weak equivalence of simplicial sets when $Z$ is the cofibrant replacement of a domain or codomain of a generating cofibration for $\mathcal{C}_{\mathbf{m}}$ and $\alpha: \mathbf{m} \rightarrow \mathbf{n}$ is a map in $\mathcal{I}$. By [14, Proposition A.5], for fixed $\alpha$ and varying $Z$, this condition is equivalent to $X_{\mathbf{m}} \rightarrow \alpha^{*}\left(X_{\mathbf{n}}\right)$ being a weak equivalence in $\mathcal{C}_{\mathbf{m}}$. The positive case is analogous. 
The argument in the last proof also implies the following statement.

COROLlary 5.5. If $Z$ is any cofibrant object in $\mathcal{C}_{\mathbf{m}}$ and $\alpha$ is a map in $\mathcal{I}$ (respectively $\mathcal{I}^{+}$), then $\alpha_{Z}$ is a weak equivalence in the absolute (respectively positive) local model structure on $\mathcal{C}^{\mathcal{I}}$.

The identifications of the model structures in the next example uses the fact that a model structure is determined by its cofibrations and fibrant objects [29, Proposition E.1.10].

EXAMPLE 5.6. (i) In the situation of Example 3.13(i), the absolute and positive local model structures are the absolute and positive $\mathcal{I}$-model structures on $\mathcal{S}^{\mathcal{I}}$; see [42, Proposition 3.2]. The weak equivalences in these model structures are called $\mathcal{I}$-equivalences and are given by the maps $X \rightarrow Y$ that induce weak homotopy equivalences $X_{h \mathcal{I}} \rightarrow Y_{h \mathcal{I}}$ on the (Bousfield-Kan) homotopy colimits of the $\mathcal{I}$-diagrams $X$ and $Y$.

(ii) In the situation of Example 3.13(ii), the absolute and positive local model structures are the respective stable model structures on $\mathrm{Sp}^{\Sigma}$; see [27, Theorem 3.4.4] and [36, Section 14].

(iii) In the situation of Example 3.13(iii), we obtain absolute and positive $\mathcal{I}$ model structures on the category $\mathcal{S}_{\mathcal{R}}^{\mathcal{I}}$ of $\mathcal{I}$-diagrams in $\mathcal{S}_{\mathcal{R}}$. They can also be constructed by identifying $\mathcal{S}_{\mathcal{R}}^{\mathcal{I}}$ with the category of retractive objects in $\mathcal{S}^{\mathcal{I}}$ and applying the argument in the proof of Proposition 2.5 to the $\mathcal{I}$ model structures on $\mathcal{S}^{\mathcal{I}}$. Moreover, the absolute local model structure on $\mathcal{S}_{\mathcal{R}}^{\mathcal{I}}$ coincides with the hocolim model structure obtained from [14, Theorem 5.2].

(vi) In the situation of Example 3.13(iv), the absolute local model structure on $\mathcal{S}_{X}^{\mathcal{I}}$ corresponds to the model structure on the category $\left(\mathcal{S}^{\mathcal{I}}\right)_{X}$ of $\mathcal{I}$-spaces over and under $X$ induced by the $\mathcal{I}$-model structure on $\mathcal{S}^{\mathcal{I}}$. To see this, we note that the explicit description of the $\mathcal{I}$-fibrations in $\mathcal{S}^{\mathcal{I}}$ in terms of homotopy cartesian squares [42, Section 3.1] implies that the fibrant objects in $\left(\mathcal{S}^{\mathcal{I}}\right)_{X}$ match the local objects in $\mathcal{S}_{X}^{\mathcal{I}}$.

\subsection{Local model structures on symmetric spectra in retractive spaces.} Next we consider the category of symmetric spectra in retractive spaces $\mathrm{Sp}_{\mathcal{R}}^{\Sigma}$ introduced in Definition 3.14. An object is fibrant in the resulting absolute (respectively positive) local model structure if and only if it is absolute (respectively positive) level fibrant and the adjoint structure maps $(E, X)(\mathbf{m}) \rightarrow \operatorname{Hom}_{\mathcal{R}}\left(\left(S^{\mathbf{n}-\alpha}, *\right),(E, X)(\mathbf{n})\right)$ are weak equivalences in $\mathcal{S}_{\mathcal{R}}$ for 
all $\alpha$ in $\mathcal{I}$ (respectively $\mathcal{I}^{+}$). In view of the definition of the cotensor in (2.23), the latter condition means that the horizontal maps in the following diagram are required to be weak equivalences:

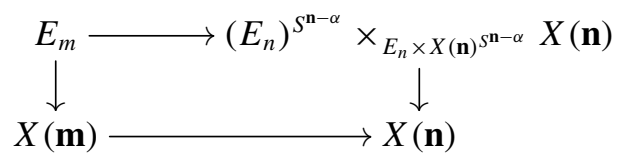

Our absolute and positive local model structures on $\mathrm{Sp}_{\mathcal{R}}^{\Sigma}$ coincide with the corresponding model structures on symmetric spectra in $\mathcal{S}_{\mathcal{R}}$ considered elsewhere in the literature (compare, for example, $[18,26]$ ).

Proposition 5.9. The weak equivalences in the absolute and positive local model structures on $\mathrm{Sp}_{\mathcal{R}}^{\Sigma}$ coincide.

Proof. This follows from [18, Theorem 10].

REMARK 5.10. We resist from calling the model structures from Proposition 5.2 stable since they are not necessarily stable in the sense that suspension becomes invertible on the homotopy category. In fact, $\mathcal{S}^{\mathcal{I}}$ and $\mathrm{Sp}_{\mathcal{R}}^{\Sigma}$ have no zero objects and cannot be stable in the latter sense.

Proposition 5.11. The absolute and positive local model structures on $\mathrm{Sp}_{\mathcal{R}}^{\Sigma}$ satisfy the pushout product axiom with respect to $\bar{\wedge}$.

Proof. The absolute case follows from [26, Theorem 8.11]. Using Proposition 5.9, the positive case follows from the pushout product axiom for the absolute local and the positive level model structure.

LEMMA 5.12. The left adjoint functors $\iota_{t}, \iota_{b}, \mathbb{S}_{\mathcal{R}}^{\mathcal{I}}$, and $\pi_{b}$ introduced in (3.17) are left Quillen functors with respect to the absolute and positive local model structures.

Proof. For the functor $\mathbb{S}_{\mathcal{R}}^{\mathcal{I}}$, we observe that $\mathbb{S}_{\mathcal{R}}^{\mathcal{I}}\left[F_{\mathbf{n}}^{\mathcal{S}}{ }^{\mathcal{I}}(\alpha ! Z)\right] \cong F_{\mathbf{n}}^{\mathrm{Sp}} \bar{R}_{\mathcal{R}}^{\Sigma}\left(\alpha_{!}\left(Z \bar{\wedge} S^{m}\right)\right)$ where the first $\alpha_{!}$is part of the pseudofunctor defining $\mathcal{S}_{\mathcal{R}}^{\mathcal{I}}$ and the second is part of the pseudofunctor defining $\operatorname{Sp}_{\mathcal{R}}^{\Sigma}$. Since $Z \bar{\wedge} S^{m}$ is cofibrant in $\mathcal{S}_{\mathcal{R}}$ if $Z$ is, Corollary 5.5 and [23, Proposition 3.3.18(1)] show that $\mathbb{S}_{\mathcal{R}}^{\mathcal{I}}$ is left Quillen. The other cases are analogous (but easier). 
COROLlaRY 5.13. Both $\left(\mathbb{S}_{b}^{\mathcal{I}}, \pi_{b}\right)$ and $\left(\pi_{b}, \mathbb{S}_{b}^{\mathcal{I}}\right)$ are Quillen adjunctions with respect to the absolute and positive local model structures.

The absolute and positive $\mathcal{I}$-model structures on $\mathcal{S}^{\mathcal{I}}$ give rise to injective model structures on $\operatorname{Ar}\left(\mathcal{S}^{\mathcal{I}}\right)$ where a map is a cofibration or weak equivalence if and only if its two components have this property in $\mathcal{S}^{\mathcal{I}}$.

LEMMA 5.14. The adjunction $\iota_{\mathrm{ar}}: \operatorname{Ar}\left(\mathcal{S}^{\mathcal{I}}\right) \rightleftarrows \mathcal{S}_{\mathcal{R}}^{\mathcal{I}}: \pi_{\mathrm{ar}}$ from (4.8) is a Quillen adjunction with respect to the absolute or positive model structures on $\operatorname{Ar}\left(\mathcal{S}^{\mathcal{I}}\right)$ and the respective local model structures on $\mathcal{S}_{\mathcal{R}}^{\mathcal{I}}$.

5.15. The local model structures on $X$-relative symmetric spectra. Let $X$ be an $\mathcal{I}$-space. Then Proposition 5.2 gives rise to absolute and positive local model structures on the category $\mathrm{Sp}_{X}^{\Sigma}$ of $X$-relative symmetric spectra. When $K$ is a space, then these local model structures on $\mathrm{Sp}_{K}^{\Sigma}=\mathrm{Sp}_{\text {const }_{\mathcal{I}}}^{\Sigma}$ correspond to the absolute and positive stable model structure on $\operatorname{Sp}^{\Sigma}\left(\mathcal{S}_{K}, S^{1}\right)$, and the fibrant objects are the absolute (respectively positive) $\Omega$-spectra in the latter category. For a general base $\mathcal{I}$-space $X$, an object $(E, X)$ is fibrant in the absolute (respectively positive) local model structure on $\operatorname{Sp}_{X}^{\Sigma}$ if and only if it is absolute (respectively positive) level fibrant and the square (5.8) is homotopy cartesian for all $\alpha$ in $\mathcal{I}$ (respectively $\mathcal{I}^{+}$). Although their base $\mathcal{I}$-space may not be constant, we think of the fibrant objects as fiberwise (positive) $\Omega$-spectra.

REMARK 5.16. In lack of a symmetric monoidal structure on $\mathrm{Sp}_{X}^{\Sigma}$, we cannot directly apply [18, Theorem 10] to show that the weak equivalences in the absolute and positive local model structures coincide. We will derive this from the corresponding result for $\mathrm{Sp}_{\mathcal{R}}^{\Sigma}$ in Corollary 6.5.

LEMMA 5.17. If $f: X \rightarrow Y$ is a map of $\mathcal{I}$-spaces, then $f_{!}: \operatorname{Sp}_{X}^{\Sigma} \rightleftarrows \operatorname{Sp}_{Y}^{\Sigma}: f^{*}$ is a Quillen adjunction with respect to the absolute and positive local model structures. If $f: X \rightarrow Y$ is an absolute (respectively positive) level equivalence, then $\left(f_{!}, f^{*}\right)$ is a Quillen equivalence with respect to the absolute (respectively positive) local model structures.

Proof. An adjunction argument shows that the cobase changes $f_{!}: \operatorname{Sp}_{X}^{\Sigma} \rightarrow \operatorname{Sp}_{Y}^{\Sigma}$ and $f_{!}: \mathcal{S}_{X(\mathbf{m})} \rightarrow \mathcal{S}_{Y(\mathbf{m})}$ commute with the free functors. Since the standard generating cofibrations for $\mathcal{S}_{X(\mathbf{m})}$ have cofibrant domains [24], it follows from Corollary 5.5 and [23, Proposition 3.3.18(1)] that $f_{\text {! }}$ is left Quillen with respect to the local model structures. For the Quillen equivalence statement, it is by [25, Proposition 1.1.13] sufficient that the derived unit and counit of the adjunction $\left(f_{!}, f^{*}\right)$ are natural weak equivalences. For the derived counit, the claim follows 
because $\left(f_{!}, f^{*}\right)$ is a Quillen equivalence in all (respectively all positive) levels. For the derived unit, it is sufficient to show that $(E, X) \rightarrow f^{*}\left(f_{!}(E, X)^{\text {loc-fib }}\right)$ is a weak equivalence when $(E, X)$ is both cofibrant and fibrant in the local model structure. The fibrancy condition implies that a level fibrant replacement of the object $f_{!}(E, X)$ is already a fibrant replacement in the local model structure, and so the map in question is an absolute (respectively a positive) level equivalence because $\left(f_{!}, f^{*}\right)$ is a Quillen equivalence in all (respectively all positive) levels.

The less obvious result that $\left(f_{!}, f^{*}\right)$ is already a Quillen equivalence if $f$ is an $\mathcal{I}$-equivalence will be shown in Corollary 5.23.

LEMMA 5.18. The absolute and positive local model structures on $\operatorname{Sp}_{X}^{\Sigma}$ are simplicial.

Proof. This follows from Proposition 4.16 and [23, Theorem 4.1.1(4)].

Proposition 5.19. The absolute and positive local model structures on $\mathrm{Sp}_{X}^{\Sigma}$ are stable.

Proof. Since the positive case is analogous, we only discuss the absolute case.

The suspension on $\mathrm{Sp}_{X}^{\Sigma}$ is the based tensor with $S^{1}$, which is isomorphic to the functor $F_{0}^{\mathrm{Sp}_{\mathcal{R}}^{\Sigma}}\left(S^{1}\right) \bar{\wedge}-: \mathrm{Sp}_{X}^{\Sigma} \rightarrow \mathrm{Sp}_{X}^{\Sigma}$ arising from restricting the $\bar{\wedge}$-product on $\mathrm{Sp}_{\mathcal{R}}^{\Sigma}$. The latter functor is a left adjoint since $\mathrm{Sp}_{\mathcal{R}}^{\Sigma}$ is a closed monoidal, and left Quillen by the previous lemma. We need to show that it induces an equivalence on homotopy categories. The inclusion $\iota: \mathbf{0} \rightarrow \mathbf{1}$ induces a map

$$
i: F_{1}^{\mathcal{S}^{\mathcal{I}}}(*) \otimes X \rightarrow F_{0}^{\mathcal{S}^{\mathcal{I}}}(*) \otimes X \stackrel{\cong}{\rightarrow} X
$$

This $i$ is an $\mathcal{I}$-equivalence by [42, Proposition 8.2]. We consider the composite

$$
D=i_{!}\left(F_{1}^{\mathrm{Sp}_{\mathcal{R}}^{\Sigma}}\left(S^{0}\right) \bar{\wedge}-\right): \mathrm{Sp}_{X}^{\Sigma} \rightarrow \mathrm{Sp}_{X}^{\Sigma}
$$

Lemma 4.6 implies that both composites of $F_{\mathbf{0}}^{\mathrm{Sp}_{\mathcal{R}}^{\Sigma}}\left(S^{1}\right) \bar{\wedge}-$ and $D$ are isomorphic to the functor $I=i_{!}\left(F_{1}^{\mathrm{Sp}_{\mathcal{R}}^{\Sigma}}\left(S^{1}\right) \bar{\wedge}-\right): \operatorname{Sp}_{X}^{\Sigma} \rightarrow \operatorname{Sp}_{X}^{\Sigma}$. The functors $D$ and $I$ are left adjoint since the symmetric monoidal structure of $\operatorname{Sp}_{\mathcal{R}}^{\Sigma}$ is closed. They are left Quillen with respect to the absolute level model structures by Lemma 3.23, Corollary 3.30, and Proposition 4.5. To see that they are left Quillen with respect to the absolute local model structures, we note that Lemmas 3.28 and 4.6 as well 
as the isomorphism (4.4) give rise to natural isomorphisms

$$
\begin{aligned}
& i_{!}\left(F_{\mathbf{1}}^{\mathrm{Sp}_{\mathcal{R}}^{\Sigma}}\left(S^{k}\right) \bar{\wedge} F_{\mathbf{m}}^{\mathrm{Sp}_{X}^{\Sigma}}(Z)\right) \cong\left(F_{1 \sqcup \mathbf{m}}^{S^{\mathcal{I}}}(X(\mathbf{m})) \rightarrow X\right) ! F_{\mathbf{1}}^{\mathrm{Sp}_{\mathcal{R}}^{\Sigma}}\left(S^{k} \bar{\wedge} Z\right) \\
& \left.\cong F_{\mathbf{1} \mathbf{m}}^{\mathrm{Sp}_{X}^{\Sigma}}\left((\iota \sqcup \mathbf{m})_{*}: X(\mathbf{m}) \rightarrow X(\mathbf{1} \sqcup \mathbf{m})\right)_{!}\left(S^{k} \bar{\wedge} Z\right)\right) .
\end{aligned}
$$

Hence, the functor $i_{!}\left(F_{1}^{\mathrm{Sp}_{\mathcal{R}}^{\Sigma}}\left(S^{k}\right) \bar{\wedge}-\right)$ sends the maps $\alpha_{Z}$ used to form the local model structures to $(1 \sqcup \alpha)_{Y}$ with $Y=\left((\iota \sqcup \mathbf{m})_{*}: X(\mathbf{m}) \rightarrow X(\mathbf{1} \sqcup \mathbf{m})\right)_{!}\left(S^{k} \bar{\wedge} Z\right)$, and $(1 \sqcup \alpha)_{Y}$ is a local equivalence by Corollary 5.5. Combining this identification for $k=0$ and $k=1$ with [23, Proposition 3.3.18(1)] implies that $D$ and $I$ are left Quillen with respect to the absolute local model structures.

Since $F_{1}^{\mathcal{S}^{\mathcal{I}}}(*) \rightarrow F_{\mathbf{0}}^{\mathcal{S}^{\mathcal{I}}}(*)$ is the map of base spaces underlying $\iota_{S^{1}}: F_{1}^{\mathrm{Sp}_{\mathcal{R}}^{\Sigma}}\left(S^{1}\right) \rightarrow$ $F_{\mathbf{0}}^{\mathrm{Sp}_{\mathcal{R}}^{\Sigma}}\left(S^{0}\right)=\mathbb{S}$, the latter map and the identification (3.27) induce a natural transformation $I \rightarrow$ id of endofunctors of $\operatorname{Sp}_{X}^{\Sigma}$. Since both functors are left Quillen, a cell induction argument reduces the claim to showing that $I \rightarrow$ id is a local equivalence when evaluated on the domains and codomains of generating cofibrations. To see this, we note that the isomorphism (5.20) implies that the evaluation of $I \rightarrow$ id on $F_{\mathbf{m}}^{\mathrm{Sp}_{X}^{\Sigma}}(Z)$ is isomorphic to the map $(\iota \sqcup \mathbf{m})_{Z}$, which is a local equivalence by construction.

We now consider the diagram

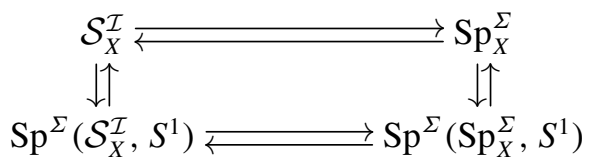

where the vertical adjunctions are the stabilizations [26, Theorem 9.1] and the horizontal left adjoints are given by $\mathbb{S}_{\mathcal{R}}^{\mathcal{I}}$ and its induced functor on symmetric spectrum objects. The left adjoints and the right adjoints commute up to isomorphism.

LEMMA 5.21. With respect to the absolute and positive local model structures, the two adjunctions $\operatorname{Sp}_{X}^{\Sigma} \rightleftarrows \operatorname{Sp}^{\Sigma}\left(\operatorname{Sp}_{X}^{\Sigma}, S^{1}\right)$ and $\operatorname{Sp}^{\Sigma}\left(\mathcal{S}_{X}^{\mathcal{I}}, S^{1}\right) \rightleftarrows \operatorname{Sp}^{\Sigma}\left(\operatorname{Sp}_{X}^{\Sigma}, S^{1}\right)$ are Quillen equivalences. In particular, $\mathbb{S}_{\mathcal{R}}^{\mathcal{I}}$ models the stabilization of $\mathcal{S}_{X}^{\mathcal{I}}$.

Proof. For $\mathrm{Sp}_{X}^{\Sigma} \rightleftarrows \operatorname{Sp}^{\Sigma}\left(\mathrm{Sp}_{X}^{\Sigma}, S^{1}\right)$, this follows from Proposition 5.19 and [26, Theorem 9.1]. For the second adjunction, we note that the category of symmetric spectrum objects in $\mathcal{S}_{X}^{\mathcal{I}}$ is equivalent to the section category of the Quillen $\mathcal{I}$-category $\mathbf{m} \mapsto \operatorname{Sp}^{\Sigma}\left(\mathcal{S}_{X(\mathbf{m})}, S^{1}\right)$ whose structure maps are induced by those discussed in Example 5.6(iv). Inspecting the cofibrations and fibrant objects, it 
follows that the stable model structure on $\operatorname{Sp}^{\Sigma}\left(\mathcal{S}_{X}^{\mathcal{I}}, S^{1}\right)$ corresponds to the local model structure associated with this Quillen $\mathcal{I}$-category where the categories $\operatorname{Sp}^{\Sigma}\left(\mathcal{S}_{X(\mathbf{m})}, S^{1}\right)$ are equipped with the stable model structure. Analogously, we can identify $\operatorname{Sp}^{\Sigma}\left(\operatorname{Sp}_{X}^{\Sigma}, S^{1}\right)$ with the section category of $\mathbf{m} \mapsto \operatorname{Sp}^{\Sigma}\left(\mathcal{S}_{X(\mathbf{m})}, S^{1}\right)$ where now the structure maps are the spectrifications of the structure maps (3.21) for $\mathrm{Sp}_{X}^{\Sigma}$. Again, the stable model structure corresponds to the local model structure on the section category. Under these identifications, the adjunction in question is induced in level $\mathbf{m}$ by the left adjoints $S^{\mathbf{m}} \bar{\wedge}-: \operatorname{Sp}^{\Sigma}\left(\mathcal{S}_{X(\mathbf{m})}, S^{1}\right) \rightarrow \operatorname{Sp}^{\Sigma}\left(\mathcal{S}_{X(\mathbf{m})}, S^{1}\right)$ in the way explained in Lemma 3.15. By stability, the latter functor participates in a Quillen equivalence, and the claim follows by a similar argument as in the proof of Lemma 5.17.

REMARK 5.22. The lemma implies that the model category $\operatorname{Sp}_{X}^{\Sigma}$ we are interested in is also equivalent to $\operatorname{Sp}^{\Sigma}\left(\mathcal{S}_{X}^{\mathcal{I}}, S^{1}\right)$. However, the latter category is more complicated in that it has separate $\mathcal{I}$ - and spectrum directions, and it is less suited for the approach to Thom spectra in Section 9.1 and the analysis of parametrized spectra carried out in [21, Section 5].

Corollary 5.23. If $f: X \rightarrow Y$ is an $\mathcal{I}$-equivalence, then $f_{!}: \operatorname{Sp}_{X}^{\Sigma} \rightleftarrows \operatorname{Sp}_{Y}^{\Sigma}: f^{*}$ is a Quillen equivalence with respect to the absolute and positive local model structures.

Proof. We know from Lemma 5.17 that $\left(f_{!}, f^{*}\right)$ is a Quillen adjunction. By properness of the $\mathcal{I}$-model structures on $\mathcal{S}^{\mathcal{I}}$ (see [42, Proposition 3.2]) and the discussion in Example 5.6 (iv), it follows that $f$ induces a Quillen equivalence $\mathcal{S}_{X}^{\mathcal{I}} \rightleftarrows \mathcal{S}_{Y}^{\mathcal{I}}$. By [26, Theorem 9.3], this Quillen equivalence induces a Quillen equivalence on the stabilization. The claim follows by the last lemma and 2-outof-3 for Quillen equivalences.

Let again $X_{h \mathcal{I}}=\operatorname{hocolim}_{\mathcal{I}} X$ denote the Bousfield-Kan homotopy colimit of an $\mathcal{I}$-space $X: \mathcal{I} \rightarrow \mathcal{S}$ and let $\bar{X}$ be the bar resolution of $X$, that is, the homotopy left Kan extension of $X$ along $\operatorname{id}_{\mathcal{I}}$. Then the adjoint of the isomorphism $\operatorname{colim}_{\mathcal{I}}(\bar{X}) \cong X_{h \mathcal{I}}$ and the canonical map $\bar{X} \rightarrow X$ provide a zig-zag of $\mathcal{I}$ equivalences const $X_{\mathcal{I}} \leftarrow \bar{X} \rightarrow X$ (see, for example, [45, Section 4]). Using this, the previous corollary implies the following.

COROLlary 5.24. Let $X$ be an $\mathcal{I}$-space. Then there is a chain of Quillen equivalences relating $\mathrm{Sp}_{X}^{\Sigma}$ and $\mathrm{Sp}_{X_{h \mathcal{I}}}^{\Sigma}$ with the absolute local model structures. 


\section{Comparison with the local integral model structure}

Our next aim is to prove a version of Proposition 3.29 for the local model structures, that is, we show that the $\mathcal{I}$-model structures on $\mathcal{S}^{\mathcal{I}}$ discussed in Example 5.6(i) and the local model structures on the $\operatorname{Sp}_{X}^{\Sigma}$ assemble to the local model structures on $\mathrm{Sp}_{\mathcal{R}}^{\Sigma}$.

LEMMA 6.1. Let $X$ be an absolute (respectively a positive) $\mathcal{I}$-fibrant $\mathcal{I}$-space and let $(E, X)$ be an object in $\operatorname{Sp}_{X}^{\Sigma}$. Then $(E, X)$ is fibrant in the absolute (respectively positive) local model structure on $\mathrm{Sp}_{\mathcal{R}}^{\Sigma}$ if and only if it is fibrant in the absolute (respectively positive) local model structure on $\mathrm{Sp}_{X}^{\Sigma}$.

Proof. An object $(E, X)$ is absolute local fibrant in $\operatorname{Sp}_{\mathcal{R}}^{\Sigma}$ if and only if it is absolute level fibrant and the horizontal maps in the square (5.8) are weak equivalences for all $\alpha$ in $\mathcal{I}$. Under the assumptions on $X$, this holds if and only if $(E, X)$ is absolute level fibrant in $\operatorname{Sp}_{X}^{\Sigma}$ and (5.8) is homotopy cartesian for all $\alpha$ in $\mathcal{I}$. The positive case is analogous.

LemMA 6.2. Let $K$ be a cofibrant space, let $(U, K)$ be cofibrant in $\mathcal{S}_{K}$, and let $\alpha: \mathbf{m} \rightarrow \mathbf{n}$ be a map in $\mathcal{I}$. Then $\alpha_{(U, K)}: F_{\mathbf{n}}^{\mathrm{Sp}_{K}^{\Sigma}}\left((U, K) \bar{\wedge} S^{\mathbf{n}-\alpha}\right) \rightarrow F_{\mathbf{m}}^{\mathrm{Sp}_{K}^{\Sigma}}(U, K)$ is a local weak equivalence in $\mathrm{Sp}_{\mathcal{R}}^{\Sigma}$.

Proof. We consider the commutative diagram:

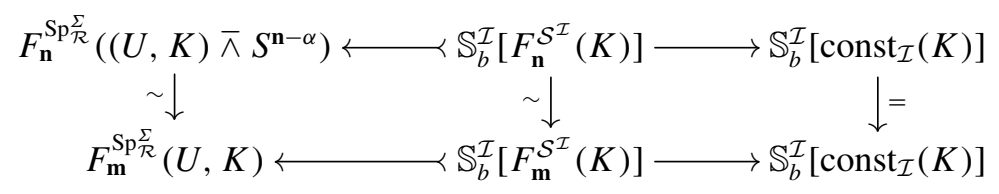

The left-hand vertical map is a local weak equivalence in $\mathrm{Sp}_{\mathcal{R}}^{\Sigma}$ by Corollary 5.5. The middle vertical map is because $F_{\mathbf{n}}^{\mathcal{S}^{\mathcal{I}}}(K) \rightarrow F_{\mathbf{m}}^{\mathcal{S}^{\mathcal{I}}}(K)$ is an $\mathcal{I}$-equivalence and $\mathbb{S}_{b}^{\mathcal{I}}$ is left Quillen by Lemma 5.12. Since $(U, K)$ and $(U, K) \bar{\wedge} S^{\mathbf{n}-\alpha}$ are cofibrant and $\mathbb{S}_{b}^{\mathcal{I}}\left[F_{\mathbf{m}}^{\mathcal{S}^{\mathcal{I}}}(K)\right] \cong F_{\mathbf{m}}^{\mathrm{Sp}_{\mathcal{R}}^{\Sigma}}(K, K)$, the left-hand horizontal maps are cofibrations in $\mathrm{Sp}_{\mathcal{R}}^{\Sigma}$. Since $\mathrm{Sp}_{\mathcal{R}}^{\Sigma}$ is left proper by Proposition 5.2, Lemma 3.28 implies the claim.

LEMMA 6.3. Let $K$ be cofibrant in $\mathcal{S}$. With respect to the absolute or positive local model structures, the inclusion functor $\mathrm{Sp}_{K}^{\Sigma} \rightarrow \mathrm{Sp}_{\mathcal{R}}^{\Sigma}$ preserves acyclic cofibrations with fibrant codomain.

Proof. Given a map $\alpha_{Z}$ in the set of maps we use to form the local model structure 
on $\mathrm{Sp}_{K}^{\Sigma}$, we use the mapping cylinder construction resulting from the simplicial structure of $\mathrm{Sp}_{K}^{\Sigma}$ to factor it into a cofibration $\alpha_{Z}^{c}$ followed by an absolute level equivalence. We let $J$ be the set of maps in $\operatorname{Sp}_{K}^{\Sigma}$ that is the union of the generating acyclic cofibrations for the absolute level model structure and the maps of the form $\alpha_{Z}^{c} \otimes i$ where $i=\left(\partial D^{n} \rightarrow \Delta^{n}\right)$ runs through the generating cofibrations sSet and $\alpha_{Z}$ runs through the maps we are localizing at. Writing $X=$ const $_{\mathcal{I}} K$, an object $(E, X)$ is fibrant in $\operatorname{Sp}_{K}^{\Sigma}$ if and only if $(E, X) \rightarrow \mathbb{S}_{b}^{\mathcal{I}}[X]$ has the right lifting property with respect to $J$ (compare [23, Proposition 4.2.4] for an analogous statement using cosimplicial resolutions). The domains of the maps in $J$ are small relative to $J$-cell complexes because this property is inherited from the cofibrantly generated model category $\mathcal{S}_{K}$ (and preserved by forming the mapping cylinder). Hence, we can apply the small object argument to see that the fibrant replacement in the local model structure on $\mathrm{Sp}_{K}^{\Sigma}$ is the retract of a $J$-cell complex.

By Lemma 6.2 and Proposition 5.11, the maps in $J$ are acyclic cofibrations in the absolute local model structure on $\mathrm{Sp}_{\mathcal{R}}^{\Sigma}$. Since the inclusion $\mathrm{Sp}_{X}^{\Sigma} \rightarrow \operatorname{Sp}_{\mathcal{R}}^{\Sigma}$ preserves pushouts and filtered colimits, it follows that $J$-cell complexes are also acyclic cofibrations in $\operatorname{Sp}_{\mathcal{R}}^{\Sigma}$. The claim follows because the fibrant objects in $\mathrm{Sp}_{K}^{\Sigma}$ and $\mathrm{Sp}_{\mathcal{R}}^{\Sigma}$ coincide by Lemma 6.1.

Proposition 6.4. A map in $\mathrm{Sp}_{X}^{\Sigma}$ is a weak equivalence in the absolute or positive local model structure if and only if it is so as a map in $\mathrm{Sp}_{\mathcal{R}}^{\Sigma}$.

Proof. We consider a map $\varphi:(E, X) \rightarrow\left(E^{\prime}, X\right)$ and prove the claim by gradually allowing more and more general cases. When $X=$ const $_{\mathcal{I}} K$ is the constant $\mathcal{I}$ diagram on a cofibrant space $K$ and both $(E, X)$ and $\left(E^{\prime}, X\right)$ are locally fibrant in $\mathrm{Sp}_{K}^{\Sigma}$, then they are also locally fibrant as objects in $\mathrm{Sp}_{\mathcal{R}}^{\Sigma}$ by Lemma 6.1, and the claim follows since in both categories, weak equivalences between fibrant objects are level equivalences. When $(E, X)$ and $\left(E^{\prime}, X\right)$ are not necessarily fibrant in $\mathrm{Sp}_{K}^{\Sigma}$, we apply the fibrant replacement in $\mathrm{Sp}_{K}^{\Sigma}$ to $\varphi$ and use Lemma 6.3 to see that it is also a fibrant replacement in $\mathrm{Sp}_{\mathcal{R}}^{\Sigma}$. Hence, the claim reduces to the previous case.

In the next step, we assume that $X$ is absolute (respectively positive) cofibrant as an $\mathcal{I}$-space. Setting $K=\operatorname{colim}_{\mathcal{I}} X$, the adjunction counit provides an $\mathcal{I}$ equivalence $f: X \rightarrow$ const $_{\mathcal{I}} K$. Now given a map $\varphi:(E, X) \rightarrow\left(E^{\prime}, X\right)$ of cofibrant objects in $\mathrm{Sp}_{X}^{\Sigma}$, we apply Corollary 5.23 to see that $\left(f_{!}, f^{*}\right)$ is a Quillen equivalence and deduce that $\varphi$ is a local weak equivalence in $\operatorname{Sp}_{X}^{\Sigma}$ if and only if $f_{!}(\varphi)$ is a local weak equivalence in $\operatorname{Sp}_{K}^{\Sigma}$. Left properness of the level model structure on $\mathrm{Sp}_{\mathcal{R}}^{\Sigma}$, the identification (3.27), and Corollary 5.13 imply that $\varphi$ is a local weak equivalence in $\operatorname{Sp}_{\mathcal{R}}^{\Sigma}$ if and only if $f_{!}(\varphi)$ is. So we have reduced the claim to the previous step. Since the cofibrant replacement in $\operatorname{Sp}_{X}^{\Sigma}$ is a level 
equivalence, we may drop the cofibrancy assumption on $(E, X)$ and $\left(E^{\prime}, X\right)$ in the previous argument.

In the last step, we consider a general $X$ and let $f: X^{c} \rightarrow X$ be an absolute (respectively positive) acyclic fibration with absolute (respectively positive) cofibrant domain. Since $f$ is a level equivalence and $\mathcal{S}$ is proper, $\left(f_{!}, f^{*}\right)$ is a Quillen equivalence with respect to the level model structures. Hence, our test map $\varphi:(E, X) \rightarrow\left(E^{\prime}, X\right)$ is level equivalent to the image of a map of cofibrant objects $\varphi^{c}:\left(E^{c}, X^{c}\right) \rightarrow\left(E^{\prime c}, X^{c}\right)$ in $\operatorname{Sp}_{X^{c}}^{\Sigma}$ under $f_{!}$. Since $\left(f_{!}, f^{*}\right)$ is a Quillen equivalence with respect to the local model structures by Corollary 5.13, $\varphi$ is a local equivalence in $\operatorname{Sp}_{X}^{\Sigma}$ if and only if $\varphi^{c}$ is a local equivalence in $\operatorname{Sp}_{X^{c}}^{\Sigma}$. Since the level model structures on $\operatorname{Sp}_{\mathcal{R}}^{\Sigma}$ are right proper by Proposition 3.10, $\varphi$ and $\varphi^{c}$ are level equivalent in $\mathrm{Sp}_{\mathcal{R}}^{\Sigma}$. This reduces the general claim to the previous case.

COROLlary 6.5. The weak equivalences in the absolute and the positive local model structures on $\mathrm{Sp}_{X}^{\Sigma}$ coincide.

Proof. This follows by combining Propositions 5.9 and 6.4.

Corollary 6.6. Let $f: X \rightarrow Y$ be a map of $\mathcal{I}$-spaces. If $f$ is an acyclic cofibration (respectively acyclic fibration) in the absolute $\mathcal{I}$-model structure, then $f_{!}: \mathrm{Sp}_{X}^{\Sigma} \rightarrow \operatorname{Sp}_{Y}^{\Sigma}$ (respectively $f^{*}: \mathrm{Sp}_{Y}^{\Sigma} \rightarrow \mathrm{Sp}_{X}^{\Sigma}$ ) preserves weak equivalences of the local model structures. An analogous statement holds in the positive case.

Proof. If $f: X \rightarrow Y$ is an acyclic cofibration, then $\mathbb{S}_{b}^{\mathcal{I}}[f]$ is an acyclic cofibration in the local model structure on $\operatorname{Sp}_{\mathcal{R}}^{\Sigma}$ by Corollary 5.13. The claim follows by the first isomorphism in (3.27) and Proposition 6.4. The statement about $f^{*}$ can be proved by arguing in a dual way.

Applying Definition 2.17 to the pseudofunctor $X \mapsto \mathrm{Sp}_{X}^{\Sigma}$ from (3.25), the absolute (respectively positive) $\mathcal{I}$-model structure and the absolute (respectively positive) local model structure on the $\mathrm{Sp}_{X}^{\Sigma}$ give rise to absolute (respectively positive) local integral cofibrations, fibrations, and weak equivalences on the Grothendieck construction.

THEOREM 6.7. These classes of maps form an absolute (respectively positive) integral local model structure on the Grothendieck construction. Under the equivalence with $\mathrm{Sp}_{\mathcal{R}}^{\Sigma}$, it coincides with the absolute (respectively positive) local model structures on $\mathrm{Sp}_{\mathcal{R}}^{\Sigma}$.

Proof. Combining Lemma 5.17, Corollary 5.23, and Corollary 6.6, the existence 
of the integral model structure follows from [20, Theorem 3.0.12]. For the comparison, we note that the cofibrations and fibrant objects of the two model structures coincide by Proposition 3.29 and Lemma 6.1. Hence, the claim follows from [29, Proposition E.1.10].

The last theorem and the definition of the integral model structure imply the next two statements.

COROLLARY 6.8. A map in $\mathrm{Sp}_{X}^{\Sigma}$ is a cofibration, fibration, or weak equivalence in the absolute or positive local model structure if and only if it is so as a map in $\mathrm{Sp}_{\mathcal{R}}^{\Sigma}$.

Corollary 6.9. Let $(E, X) \rightarrow(F, Y)$ be a map in $\operatorname{Sp}_{\mathcal{R}}^{\Sigma}$ with $f: X \rightarrow Y$ as map of base $\mathcal{I}$-spaces. Then the following are equivalent:

(i) The map $(E, X) \rightarrow(F, Y)$ is a local weak equivalence in $\mathrm{Sp}_{\mathcal{R}}^{\Sigma}$.

(ii) $f$ is an $\mathcal{I}$-equivalence and a cofibrant replacement $\left(E^{c}, X\right) \rightarrow(E, X)$ in $\mathrm{Sp}_{X}^{\Sigma}$ induces a local weak equivalence $\left(f_{!}\left(E^{c}\right), Y\right) \rightarrow\left(f_{!}(E), Y\right) \rightarrow(F, Y)$ in $\mathrm{Sp}_{Y}^{\Sigma}$.

(iii) $f$ is an $\mathcal{I}$-equivalence and a fibrant replacement $(F, Y) \rightarrow\left(F^{f}, Y\right)$ in $\mathrm{Sp}_{Y}^{\Sigma}$ induces a local weak equivalence $(E, X) \rightarrow\left(f^{*}(F), X\right) \rightarrow\left(f^{*}\left(F^{f}\right), X\right)$ in $\mathrm{Sp}_{X}^{\Sigma}$.

We have now proved the main results about the local model structures stated in Section 1.

Proof of Theorems 1.2 and 1.3. Theorem 1.2 is a combination of Corollary 6.8, Lemma 5.17, and Corollaries 5.23 and 5.24. Theorem 1.3 is Theorem 6.7.

REMARK 6.10. Using [12, Theorem 4.2], Theorem 6.7 also implies that the functors $f_{!}$and $f^{*}$ satisfy the homotopical Beck-Chevalley condition formulated in [12, Definition 4.1].

Let $M$ be a commutative $\mathcal{I}$-space monoid. It is now easy to see that the symmetric monoidal product on $\mathrm{Sp}_{M}^{\Sigma}$ discussed in (4.12) is also compatible with the local model structures.

PROPOSITION 6.11. The category $\mathrm{Sp}_{M}^{\Sigma}$ satisfies the pushout product axiom with respect to the absolute and positive local model structures. 
Proof. Since $\mu_{!}: \mathrm{Sp}_{M \boxplus M}^{\Sigma} \rightarrow \mathrm{Sp}_{M}^{\Sigma}$ is left Quillen, Corollary 6.8 and the pushout product axiom in $\mathrm{Sp}_{\mathcal{R}}^{\Sigma}$ provide the pushout product axiom for $\mathrm{Sp}_{M}^{\Sigma}$.

By the discussion following Theorem 10.6, the previous proposition provides a symmetric monoidal model for the stabilization of the category of spaces over and under a given $E_{\infty}$ space.

REMARK 6.12. In view of Corollary 6.8, one may wonder if one can simply use the local model structure on $\mathrm{Sp}_{\mathcal{R}}^{\Sigma}$ to define the local model structures on the subcategories $\mathrm{Sp}_{X}^{\Sigma}$ and avoid many of the intermediate steps in our construction. The problem with this approach is that the factorizations in $\mathrm{Sp}_{\mathcal{R}}^{\Sigma}$ do not necessarily give rise to factorizations in $\operatorname{Sp}_{X}^{\Sigma}$. Moreover, the important property that an $\mathcal{I}$ equivalence $f: X \rightarrow Y$ induces a Quillen equivalence $f_{!}: \mathrm{Sp}_{X}^{\Sigma} \rightleftarrows \operatorname{Sp}_{Y}^{\Sigma}: f^{*}$ does not appear to be a consequence of the local model structure on $\mathrm{Sp}_{\mathcal{R}}^{\Sigma}$ since this would require a form of right properness of $\mathrm{Sp}_{\mathcal{R}}^{\Sigma}$.

6.13. Comparison of simplicial and topological variants. When developing our model structures, we allowed the underlying category of spaces $\mathcal{S}$ to be either the category sSet of simplicial sets or the category of compactly generated weak Hausdorff spaces Top. The Quillen adjunction

$$
|-| \text { : sSet } \rightleftarrows \text { Top: Sing }
$$

relating them induces an adjunction

$$
|-|: \operatorname{Sp}_{\mathcal{R}}^{\Sigma}(\mathrm{sSet}) \rightleftarrows \operatorname{Sp}_{\mathcal{R}}^{\Sigma}(\mathrm{Top}): \text { Sing }
$$

on the associated categories of symmetric spectra in retractive spaces with $|-|$ strong symmetric monoidal and Sing lax symmetric monoidal.

Proposition 6.14. The adjunction $\mathrm{Sp}_{\mathcal{R}}^{\Sigma}(\mathrm{sSet}) \rightleftarrows \mathrm{Sp}_{\mathcal{R}}^{\Sigma}$ (Top) is a Quillen equivalence with respect to the absolute and positive levels and local model structures.

Proof. This can be checked from the definitions or deduced from [26, Theorem 9.3].

Now let $X$ be an $\mathcal{I}$-diagram of simplicial sets, $Y$ an $\mathcal{I}$-diagram of topological spaces, and $|X| \rightarrow Y$ a map with adjoint $X \rightarrow \operatorname{Sing}(Y)$. Then the two composites

$$
\begin{aligned}
& \operatorname{Sp}_{X}^{\Sigma} \text { (sSet) } \stackrel{|-|}{\longrightarrow} \operatorname{Sp}_{|X|}^{\Sigma} \text { (Top) } \stackrel{(|X| \rightarrow Y)}{\longrightarrow} \operatorname{Sp}_{Y}^{\Sigma}(\text { Top }) \quad \text { and } \\
& \operatorname{Sp}_{Y}^{\Sigma} \text { (Top) } \stackrel{\text { Sing }}{\longrightarrow} \operatorname{Sp}_{\operatorname{Sing}(\mathrm{Y})}^{\Sigma}(\mathrm{sSet}) \stackrel{(X \rightarrow \operatorname{Sing}(\mathrm{Y}))^{*}}{\longrightarrow} \operatorname{Sp}_{X}^{\Sigma}(\mathrm{sSet})
\end{aligned}
$$


define an adjunction $\mathrm{Sp}_{X}^{\Sigma}$ (sSet) $\rightleftarrows \operatorname{Sp}_{Y}^{\Sigma}$ (Top). Taking $|X| \rightarrow Y$ or its adjoint to be the identity gives adjunctions $\operatorname{Sp}_{X}^{\Sigma}$ (sSet) $\rightleftarrows \operatorname{Sp}_{|X|}^{\Sigma}($ Top $)$ and $\operatorname{Sp}_{\operatorname{Sing}(Y)}^{\Sigma}(\mathrm{sSet}) \rightleftarrows$ $\mathrm{Sp}_{Y}^{\Sigma}$ (Top).

Proposition 6.15. The last two adjunctions are Quillen equivalences with respect to the absolute and positive levels and local model structures.

Proof. This follows from Proposition 6.14 and Corollaries 3.30 and 6.8.

It is also easy to check that these adjunctions respect the convolution product (4.12) if the base is a commutative $\mathcal{I}$-space monoid.

6.16. Model structures on parametrized commutative ring spectra. Next, we explain how to lift the previously constructed local model structures to commutative ring spectra, and for this, we wish to apply the general theory from [41]. Since this theory is only applicable in the simplicial setting, we shall limit ourselves to working simplicially when discussing model structures on commutative ring spectra. Thus, for the rest of this section, we specify that the underlying category $\mathcal{S}$ of spaces be the category sSet of simplicial sets. We briefly comment on the topological setting in Remark 6.21.

We write $\mathcal{C} \operatorname{Sp}_{\mathcal{R}}^{\Sigma}$ for the category of commutative ring spectra in $\mathrm{Sp}_{\mathcal{R}}^{\Sigma}$, that is, for commutative monoid objects in $\left(\mathrm{Sp}_{\mathcal{R}}^{\Sigma}, \bar{\wedge}, \mathbb{S}\right)$.

THEOREM 6.17. The category $\mathcal{C} \operatorname{Sp}_{\mathcal{R}}^{\Sigma}$ admits a positive local model structure where a map is a fibration or weak equivalence if and only if the underlying map in $\mathrm{Sp}_{\mathcal{R}}^{\Sigma}$ is.

Proof. We first note that the absolute and positive model structures can also be constructed using [41, Theorem 3.2.1]. For this, we have to show that the category $\operatorname{sSet}_{\mathcal{R}}$ of retractive simplicial sets satisfies the requirements of [41, Definition 2.1]. This holds since $\operatorname{sSet}_{\mathcal{R}}$ is locally presentable, all objects are cofibrant, the domains and codomains of the generating cofibrations are finitely presentable, and $\mathrm{sSet}_{\mathcal{R}}$ satisfies the pushout product axiom. The theorem then follows from [41, Theorem 4.1].

REMARK 6.18. In fact, the result in [41] shows that the positive local model structure on $\mathrm{Sp}_{\mathcal{R}}^{\Sigma}$ has favorable monoidal properties [41, Proposition 3.5.1] that allow it to be lifted to algebras over general colored symmetric operads. In particular, there is also a lifted model structure on associative parametrized ring spectra. 
Now let $M$ be a commutative $\mathcal{I}$-space monoid and consider the category $\operatorname{Sp}_{M}^{\Sigma}$ with the symmetric monoidal product (4.12). We write $\mathcal{C} \operatorname{Sp}_{M}^{\Sigma}$ for the category of commutative $M$-relative symmetric ring spectra, that is, the commutative monoid objects in $\left(\mathrm{Sp}_{M}^{\Sigma}, \bar{\wedge}, \mathbb{S}_{M}\right)$.

THEOREM 6.19. The category $\mathcal{C} \operatorname{Sp}_{M}^{\Sigma}$ admits a positive local model structure where a map is a fibration or weak equivalence if and only if the underlying map in $\mathrm{Sp}_{M}^{\Sigma}$ is.

The proof of this statement is more difficult because $\mathrm{Sp}_{M}^{\Sigma}$ not being equivalent to symmetric spectrum objects in some category prevents us from applying the results of [41] directly. Instead, we rely on the following lemma. To formulate it, we let $\mathbb{C}^{\mathcal{R}}: \operatorname{Sp}_{\mathcal{R}}^{\Sigma} \rightarrow \mathcal{C} \operatorname{Sp}_{\mathcal{R}}^{\Sigma}, \mathbb{C}^{M}: \operatorname{Sp}_{M}^{\Sigma} \rightarrow \mathcal{C} \operatorname{Sp}_{M}^{\Sigma}$, and $\mathbb{C}^{\mathcal{I}}: \mathcal{S}^{\mathcal{I}} \rightarrow \mathcal{C} \mathcal{S}^{\mathcal{I}}$ be the free functors which are left adjoint to the respective forgetful functors. We also note that there is a canonical inclusion functor $\mathcal{C} \operatorname{Sp}_{M}^{\Sigma} \rightarrow \mathcal{C} \operatorname{Sp}_{\mathcal{R}}^{\Sigma}$ that identifies $\mathcal{C} \operatorname{Sp}_{M}^{\Sigma}$ with the fiber of the projection functor $\pi_{b}: \mathcal{C} \operatorname{Sp}_{\mathcal{R}}^{\Sigma} \rightarrow \mathcal{C} \mathcal{S}^{\mathcal{I}}$ and that $\mathbb{S}_{b}^{\mathcal{I}}$ induces a functor $\mathbb{S}_{b}^{\mathcal{I}}: \mathcal{C S}^{\mathcal{I}} \rightarrow \mathcal{C} \operatorname{Sp}_{\mathcal{R}}^{\Sigma}$.

LEMMA 6.20. Let $(A, M)$ be an object in $\mathcal{C S p}_{M}^{\Sigma}$ and consider the maps $f:(D, M) \rightarrow(E, M)$ and $g:(D, M) \rightarrow(A, M)$ in $\operatorname{Sp}_{M}^{\Sigma}$. Let $\widetilde{g}: \mathbb{C}^{\mathcal{R}}(D, M) \rightarrow$ $(A, M)$ and $\hat{g}: \mathbb{C}^{M}(D, M) \rightarrow(A, M)$ be the adjoints of $g$ with respect to the above adjunctions. Then the cobase change of $\mathbb{C}^{M}(f)$ along $\hat{g}$ in $\mathcal{C} \operatorname{Sp}_{M}^{\Sigma}$ is isomorphic to the cobase change of $\mathbb{C}^{\mathcal{R}}(f)$ along $\widetilde{g}$ in $\mathcal{C} \mathrm{Sp}_{\mathcal{R}}^{\Sigma}$.

Proof. The underlying commutative $\mathcal{I}$-space monoid of $\mathbb{C}^{\mathcal{R}}(D, M)$ is $\mathbb{C}^{\mathcal{I}}(M)$. Inspecting the universal properties of the free functors shows that $\mathbb{C}^{M}(D, M)$ is isomorphic to $\mathbb{C}^{\mathcal{R}}(D, M) \bar{\wedge}_{\mathbb{S}_{b}^{\mathcal{I}}\left[\mathbb{C}^{\mathcal{I}}(M)\right]} \mathbb{S}_{b}^{\mathcal{I}}[M]$, the cobase change of $\mathbb{C}^{\mathcal{R}}(D, M)$ along the map given by applying $\mathbb{S}_{b}^{\mathcal{I}}$ to the adjoint $\mathbb{C}^{\mathcal{I}}(M) \rightarrow M$ of $\operatorname{id}_{M}$. Commuting pushouts in $\mathcal{C} \mathrm{Sp}_{\mathcal{R}}^{\Sigma}$, we see that

$$
\begin{aligned}
& (A, M) \bar{\wedge}_{\mathbb{C}^{M}(D, M)} \mathbb{C}^{M}(E, M)
\end{aligned}
$$

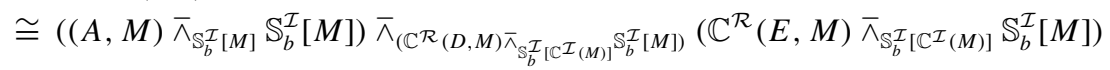

$$
\begin{aligned}
& \cong(A, M) \bar{\wedge}_{\mathbb{C}^{\mathcal{R}}(D, M)} \mathbb{C}^{\mathcal{R}}(E, M) \text {. }
\end{aligned}
$$

As the inclusion functor $\mathcal{C} \operatorname{Sp}_{M}^{\Sigma} \rightarrow \mathcal{C} \operatorname{Sp}_{\mathcal{R}}^{\Sigma}$ preserves pushouts, the claim follows.

Proof of Theorem 6.19. We apply [23, Theorem 11.3.2] to the free/forgetful adjunction $\mathbb{C}^{M}: \mathrm{Sp}_{M}^{\Sigma} \rightleftarrows \mathcal{C} \operatorname{Sp}_{M}^{\Sigma}: U$. Let $J$ be a set of generating acyclic cofibrations for the positive local model structure on $\operatorname{Sp}_{M}^{\Sigma}$ and let $\mathbb{C}^{M}(J)$ be its image under $\mathbb{C}^{M}$. The nontrivial part is to show that relative $\mathbb{C}^{M}(J)$-cell 
complexes are local equivalences. Lemma 6.20 and the fact that filtered colimits in $\mathcal{C} \operatorname{Sp}_{M}^{\Sigma}$ and $\mathcal{C} \operatorname{Sp}_{\mathcal{R}}^{\Sigma}$ are both created in $\operatorname{Sp}_{\mathcal{R}}^{\Sigma}$ imply that this follows from the corresponding property for $\mathcal{C} \operatorname{Sp}_{\mathcal{R}}^{\Sigma}$ resulting from Theorem 6.17 .

REMARK 6.21. We expect that there are analogous model structures on associative and commutative ring spectra in $\operatorname{Sp}_{\mathcal{R}}^{\Sigma}$ and $\mathrm{Sp}_{M}^{\Sigma}$ in the topological setting. However, the construction of such model structures will most likely require an elaborate analysis of $h$-cofibrations that we wish to avoid in the present paper. (Even the associative case is not an immediate consequence of [ 48 , Theorem 4.1(3)] since we do not know if the topological $\mathcal{S}_{\mathcal{R}}, \mathrm{Sp}_{\mathcal{R}}^{\Sigma}$ or $\mathrm{Sp}_{M}^{\Sigma}$ satisfies the monoid axiom.)

Nonetheless, we note that our results suffice to fibrantly replace associative or commutative parametrized ring spectra in the topological $\mathrm{Sp}_{\mathcal{R}}^{\Sigma}$ : combining Lemma 5.4 with the fact that the geometric realization $|-|:$ sSet $\rightarrow$ Top preserves fibrations and weak equivalences, it follows that $|-|: \operatorname{Sp}_{\mathcal{R}}^{\Sigma}$ (sSet) $\rightarrow \operatorname{Sp}_{\mathcal{R}}^{\Sigma}$ (Top) preserves locally fibrant objects. Thus, applying the singular complex, forming a fibrant replacement, and then passing to the realization gives a topological fibrant replacement functor for associative or commutative parametrized ring spectra that is related to the identity functor by a zig-zag of local equivalences.

Since the left adjoint functors $\iota_{t}: \mathcal{S}^{\mathcal{I}} \rightarrow \mathcal{S}_{\mathcal{R}}^{\mathcal{I}}$ and $\mathbb{S}_{\mathcal{R}}^{\mathcal{I}}: \mathcal{S}_{\mathcal{R}}^{\mathcal{I}} \rightarrow \operatorname{Sp}_{\mathcal{R}}^{\Sigma}$ from Construction 3.16 are strong symmetric monoidal, they induce adjunctions

$$
\iota_{t}: \mathcal{C} \mathcal{S}^{\mathcal{I}} \rightleftarrows \mathcal{C} \mathcal{S}_{\mathcal{R}}^{\mathcal{I}}: \pi_{t} \quad \text { and } \quad \mathbb{S}_{\mathcal{R}}^{\mathcal{I}}: \mathcal{C} \mathcal{S}_{\mathcal{R}}^{\mathcal{I}} \rightleftarrows \mathcal{C} \operatorname{Sp}_{\mathcal{R}}^{\Sigma}: \Omega_{\mathcal{R}}^{\mathcal{I}} .
$$

LEMMA 6.22. These adjunctions and their composite $\mathbb{S}_{t}^{\mathcal{I}}: \mathcal{C} \mathcal{S}^{\mathcal{I}} \rightleftarrows \mathcal{C} \operatorname{Sp}_{\mathcal{R}}^{\Sigma}: \Omega_{t}^{\mathcal{I}}$ are Quillen adjunctions with respect to the positive local model structures.

Proof. Arguing with the right adjoints, the claim follows from Lemma 5.12.

\section{Parametrized homology and cohomology}

In this section, we define the parametrized (co)homology theories associated with a parametrized spectrum that were outlined in Section 1. Concrete examples arise from the universal line bundle (see Section 8 and Proposition 10.18) and the twisted $K$-theory spectra studied in [21].

Key ingredients for the definition of parametrized (co)homology are the adjoints of the derived restriction that we discuss now. If $f: Y \rightarrow X$ is a map of $\mathcal{I}$-spaces, then $f^{*}: \mathrm{Sp}_{X}^{\Sigma} \rightarrow \mathrm{Sp}_{Y}^{\Sigma}$ is right Quillen with respect to the absolute local model structures and thus induces a right derived functor $\mathbb{R} f^{*}: \operatorname{Ho}\left(\operatorname{Sp}_{X}^{\Sigma}\right) \rightarrow$ $\operatorname{Ho}\left(\operatorname{Sp}_{Y}^{\Sigma}\right)$ with left adjoint $\mathbb{L} f_{!}: \operatorname{Ho}\left(\operatorname{Sp}_{Y}^{\Sigma}\right) \rightarrow \operatorname{Ho}\left(\operatorname{Sp}_{X}^{\Sigma}\right)$. 
Proposition 7.1. The functor $\mathbb{R} f^{*}: \operatorname{Ho}\left(\operatorname{Sp}_{X}^{\Sigma}\right) \rightarrow \operatorname{Ho}\left(\operatorname{Sp}_{Y}^{\Sigma}\right)$ is also a left adjoint.

The proposition will be proved at the end of this section.

DEFINITION 7.2. We write $\mathbb{R} f_{*}: \operatorname{Ho}\left(\operatorname{Sp}_{Y}^{\Sigma}\right) \rightarrow \operatorname{Ho}\left(\operatorname{Sp}_{X}^{\Sigma}\right)$ for the right adjoint of $\mathbb{R} f^{*}$ that results from the previous proposition. When $X=*$, we use the notation $\mathbb{R} \Gamma$ for the functor $\mathbb{R}(Y \rightarrow *)_{*}: \operatorname{Ho}\left(\operatorname{Sp}_{Y}^{\Sigma}\right) \rightarrow \operatorname{Ho}\left(\operatorname{Sp}^{\Sigma}\right)$ and the notation $\mathbb{L} \Theta$ for $\mathbb{L}(Y \rightarrow *) ! \operatorname{Ho}\left(\operatorname{Sp}_{Y}^{\Sigma}\right) \rightarrow \operatorname{Ho}\left(\operatorname{Sp}^{\Sigma}\right)$.

We stress that since $f^{*}: \operatorname{Sp}_{X}^{\Sigma} \rightarrow \mathrm{Sp}_{Y}^{\Sigma}$ is, in general, not left Quillen, the functor $\mathbb{R} f_{*}$ is not the right derived functor of a right Quillen functor. In the context of topological spaces, an explicit description of $\mathbb{R} \Gamma$ in a useful special case is given in Lemma 7.27. We also point out that when working over simplicial sets, deriving the left adjoint $\Theta=(Y \rightarrow *)$ ! is not really necessary since it preserves level equivalences and thus sends local equivalences to stable equivalences.

The following statement will also be shown at the end of this section.

Proposition 7.3. Given maps of $\mathcal{I}$-spaces $f: X^{\prime} \rightarrow X$ and $g: Y^{\prime} \rightarrow Y$, the lax monoidal structure map $f^{*}(E, X) \bar{\wedge} g^{*}(F, Y) \rightarrow(f \otimes g)^{*}((E, X) \bar{\wedge}(F, Y))$ from (4.7) induces the following natural isomorphism in $\operatorname{Ho}\left(\operatorname{Sp}_{\mathcal{R}}^{\Sigma}\right)$ :

$$
\left(\mathbb{R} f^{*}\right)(E, X) \bar{\wedge}_{\mathbb{L}}\left(\mathbb{R} g^{*}\right)(F, Y) \stackrel{\cong}{\rightrightarrows}\left(\mathbb{R}\left(f \bigotimes_{\mathbb{L}} g\right)^{*}\right)\left((E, X) \bar{\wedge}_{\mathbb{L}}(F, Y)\right) .
$$

For objects $\left(E^{\prime}, X^{\prime}\right)$ and $\left(F^{\prime}, Y^{\prime}\right)$ in $\mathrm{Sp}_{\mathcal{R}}^{\Sigma}$, the isomorphism (7.4) and the units and counits for the adjunctions resulting from Proposition 7.1 applied to $f, g$, and $f \otimes g$ induce natural maps

$$
\mathbb{R} f_{*}\left(E^{\prime}, X^{\prime}\right) \bar{\bigwedge}_{\mathbb{L}} \mathbb{R} g_{*}\left(F^{\prime}, Y^{\prime}\right) \rightarrow \mathbb{R}\left(f \bigotimes_{\mathbb{L}} g\right)_{*}\left(\left(E^{\prime}, X^{\prime}\right) \bar{\bigwedge}_{\mathbb{L}}\left(F^{\prime}, Y^{\prime}\right)\right)
$$

in $\operatorname{Ho}\left(\mathrm{Sp}_{\mathcal{R}}^{\Sigma}\right)$ that are associative, commutative, and unital.

7.6. $\mathcal{I}$-spacification. To be able to define parametrized (co-)homology and Thom spectra from space level data, we now recall from [45, Section 4.2] and [6, Section 4.1] how one can pass from spaces to $\mathcal{I}$-spaces. For any $\mathcal{I}$-space $X$, there is an $\mathcal{I}$-spacification functor

$$
P_{X}: \mathcal{S} / X_{h \mathcal{I}} \rightarrow \mathcal{S}^{\mathcal{I}} / X, \quad\left(\tau: K \rightarrow X_{h \mathcal{I}}\right) \mapsto\left(P_{X}(\tau): P_{\tau}(K) \rightarrow X\right)
$$

that is a homotopy inverse of the homotopy colimit functor. We briefly recall its definition. Writing $\bar{X}$ for the homotopy left Kan extension of $X$ along $\mathrm{id}_{\mathcal{I}}$, the canonical map $t: \bar{X} \rightarrow X$ is a natural level equivalence that we refer to as 
the bar resolution. There is a natural isomorphism $\operatorname{colim}_{\mathcal{I}} \bar{X} \cong X_{h \mathcal{I}}$ with adjoint $\pi: \bar{X} \rightarrow$ const $_{\mathcal{I}} X_{h \mathcal{I}}$. A map of spaces $\tau: K \rightarrow X_{h \mathcal{I}}$ gives rise to a map of $\mathcal{I}$ spaces

$$
\text { const }_{\mathcal{I}} K \times_{\text {const }_{\mathcal{I}} X_{h \mathcal{I}}} \bar{X} \stackrel{\mathrm{pr}}{\rightarrow} \bar{X} \stackrel{t}{\rightarrow} X .
$$

This construction can be viewed as a functor $\mathcal{S} / X_{h \mathcal{I}} \rightarrow \mathcal{S}^{\mathcal{I}} / X$. In the topological case, the homotopy invariant $\mathcal{I}$-spacification functor (7.7) is defined by precomposing it with the standard Hurewicz fibrant replacement $\Gamma(\tau): \Gamma_{\tau}(K) \rightarrow X_{h \mathcal{I}}$ of $\tau$ (not to be confused with the meaning of $\Gamma$ in Definition 7.2). In the simplicial case, we replace the $\Gamma$ by the functor sending a map of simplicial sets $\tau: K \rightarrow X_{h \mathcal{I}}$ to the map $\Gamma(\tau)$ defined by the right-hand pullback square in the diagram

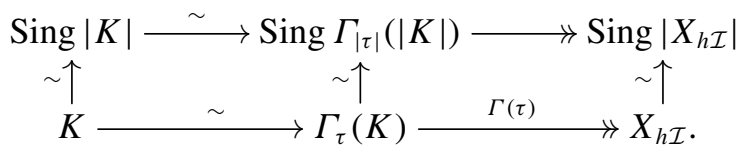

The lower left-hand map arises from the universal property of the pullback. Compared to a replacement by fibration obtained from the small object argument, this functor $\Gamma$ has the advantage of being lax monoidal and preserving operad actions. Both in the simplicial and the topological cases, the resulting $\mathcal{I}$ spacification functor $P_{X}$ then sends weak equivalences to $\mathcal{I}$-equivalences. When $M$ is a commutative $\mathcal{I}$-space monoid, $M_{h \mathcal{I}}$ is an algebra over the Barratt-Eccles operad, and $P_{M}$ preserves actions of operads augmented over the Barratt-Eccles operad and is lax monoidal.

We also note the following naturality statement for later use.

LEMMA 7.8. If $\rho: M \rightarrow N$ is a map of commutative $\mathcal{I}$-space monoids, then there is a natural map $\rho \circ P_{M}(\tau) \rightarrow P_{N}\left(\rho_{h \mathcal{I}} \circ \tau\right)$ of spaces over $N$ that is an $\mathcal{I}$-equivalence if $\rho$ is.

7.9. Parametrized homology and cohomology. To define the parametrized (co)homology groups associated with a parametrized spectrum $(E, X)$, we use the $\mathcal{I}$-spacification discussed in (7.7). Given a map $\tau: K \rightarrow X_{h \mathcal{I}}$, we use the shorthand notation $\tau_{\mathcal{I}}=P_{X}(\tau): P_{\tau}(K) \rightarrow X$ and write $\mathbb{R} \tau_{\mathcal{I}}^{*}: \operatorname{Ho}\left(\operatorname{Sp}_{X}^{\Sigma}\right) \rightarrow$ $\operatorname{Ho}\left(\operatorname{Sp}_{P_{\tau}(K)}^{\Sigma}\right)$ for the induced functor. Moreover, for any $\mathcal{I}$-space $Y$, the functors $\mathbb{L} \Theta, \mathbb{R} \Gamma: \operatorname{Ho}\left(\operatorname{Sp}_{Y}^{\Sigma}\right) \rightarrow \operatorname{Ho}\left(\operatorname{Sp}^{\Sigma}\right)$ denote the left and right adjoints, respectively, of $\mathbb{R}(Y \rightarrow *)^{*}$, the derived pullback functor along the unique map $Y \rightarrow *$. 
DeFinition 7.10. For a parametrized spectrum $(E, X) \in \mathrm{Sp}_{\mathcal{R}}^{\Sigma}$, the associated parametrized (co)homology theories are given by

$$
\begin{aligned}
& (E, X)_{n}: \mathcal{S} / X_{h \mathcal{I}} \longrightarrow \mathrm{Ab}, \quad\left(\tau: K \rightarrow X_{h \mathcal{I}}\right) \longmapsto \pi_{n}(\mathbb{L} \Theta)\left(\mathbb{R} \tau_{\mathcal{I}}^{*}\right)(E, X) \quad \text { and } \\
& (E, X)^{n}: \mathcal{S} / X_{h \mathcal{I}} \longrightarrow \mathrm{Ab}, \quad\left(\tau: K \rightarrow X_{h \mathcal{I}}\right) \longmapsto \pi_{-n}(\mathbb{R} \Gamma)\left(\mathbb{R} \tau_{\mathcal{I}}^{*}\right)(E, X) .
\end{aligned}
$$

The functoriality of parametrized homology (respectively cohomology) results from the adjunction counit of $\left(\mathbb{L} f_{!}, \mathbb{R} f^{*}\right)$ (respectively the adjunction unit of $\left(\mathbb{R} f^{*}, \mathbb{R} f_{*}\right)$ ).

Instead of directly verifying the usual properties of a (co)homology theory (including the construction of relative terms and boundary maps), let us proceed by comparing these definitions with those of May and Sigurdsson [37, Definition 20.2.4], which they show satisfy a version of the usual axioms for a (co)homology theory. In Proposition 10.18, we will also compare Definition 7.10 with the $\infty$ categorical counterparts from $[1,2]$.

Proposition 7.11. For a constant $\mathcal{I}$-space $X=$ const $_{\mathcal{I}} B$, a fiberwise orthogonal spectrum $E \in \mathrm{Sp}_{B}^{\mathrm{O}}$ (in the sense of [37, Ch. 11]), and $\tau: K \rightarrow$ $X_{h \mathcal{I}}=B \times B \mathcal{I}$, there is a canonical isomorphism between our $(E, X)_{*}(K, \tau)$ and $(E, X)^{*}(K, \tau)$ on the one hand and the definitions from [37, Definition 20.2.4] applied to $K \stackrel{\tau}{\rightarrow} X_{h \mathcal{I}} \stackrel{\mathrm{pr}}{\rightarrow} B$ on the other.

REMARK 7.12. Since in the situation of the proposition, the forgetful functor $\mathrm{Sp}_{B}^{\mathrm{O}} \rightarrow \mathrm{Sp}_{X}^{\Sigma}$ induces an equivalence on homotopy categories by the conjunction of [1, Theorem B.2], our Corollary 5.24, and Lemma 10.2, we can find a weakly equivalent orthogonal spectrum to an arbitrary $(E, X) \in \mathrm{Sp}_{X}^{\Sigma}$. Thus, we can deduce the (co)homological consequences of the proposition without the orthogonality assumption. Investing Corollary 5.24 also for nonconstant $X$, we can then also deduce them for arbitrary $(E, X) \in \mathrm{Sp}_{\mathcal{R}}^{\Sigma}$. We leave the details to the reader.

Proof of Proposition 7.11. Let us first recall the definitions: For a fiberwise orthogonal parametrized spectrum $E$ over a space $B$ as in [37, Definition 11.2.3] and a map $\sigma: K \rightarrow B$, May and Sigurdsson set

$$
E_{n}(K, \sigma)=\pi_{n}(\mathbb{L} \Theta)\left(\mathbb{L} \Sigma_{B}^{\infty} K_{+} \wedge_{B}^{\mathbb{L}} E\right)
$$

and

$$
E^{n}(K, \sigma)=\pi_{-n}(\mathbb{R} \Gamma)\left(\mathbb{R} F_{B}\left(\mathbb{L} \Sigma_{B}^{\infty} K_{+}, E\right)\right),
$$

where we have adapted those functors to our notation that have occurred in our presentation. The remaining ones are $\mathbb{L} \Sigma_{B}^{\infty}(-)_{+}: \operatorname{Ho}(\mathcal{S} / B) \rightarrow \operatorname{Ho}\left(\operatorname{Sp}_{B}^{O}\right)$ 
which adds a disjoint base section and then takes the suspension spectrum, the functor $\wedge_{B}^{\mathbb{L}}: \operatorname{Ho}\left(\mathrm{Sp}_{B}^{\mathrm{O}}\right) \times \operatorname{Ho}\left(\mathrm{Sp}_{B}^{\mathrm{O}}\right) \rightarrow \operatorname{Ho}\left(\mathrm{Sp}_{B}^{\mathrm{O}}\right)$, which is the fiberwise smash product obtained by internalizing the external smash product by pullback along the diagonal, and $\mathbb{R} F_{B}: \operatorname{Ho}\left(\mathrm{Sp}_{B}^{\mathrm{O}}\right)^{\text {op }} \times \operatorname{Ho}\left(\mathrm{Sp}_{B}^{\mathrm{O}}\right) \rightarrow \operatorname{Ho}\left(\mathrm{Sp}_{B}^{\mathrm{O}}\right)$, which takes fiberwise function spectra (and has no direct counterpart in our setup; compare Remark 2.26).

Now, from [37, Proposition 13.7.4], we find $\mathbb{L} \Sigma_{B}^{\infty} K_{+} \cong \mathbb{L} \sigma_{!}\left(\mathbb{S}_{K}\right)$, where $\mathbb{S}_{K}$ denotes the trivially parametrized sphere spectrum over $K$ that is the unit for $\wedge_{K}$. Then the projection formulas [37, (11.4.5) and (11.4.6)] (verified for the derived functors in [37, Proposition 13.7.5] or investing the comparison theorem [1, Theorem B.2] also in [1, Proposition 6.8]) show that the formulas of May and Sigurdsson can be rewritten as

$$
E_{n}(K, \sigma)=\pi_{n}(\mathbb{L} \Theta)\left(\mathbb{R} \sigma^{*}\right)(E, B) \quad \text { and } \quad E^{n}(K, \sigma)=\pi_{-n}(\mathbb{R} \Gamma)\left(\mathbb{R} \sigma^{*}\right)(E, B) .
$$

But then the conjunction of our comparison in Lemma 10.3 with [1, Theorem B.2] imply that for $X=$ const $_{\mathcal{I}} B$, we may interpret the above formulas in our categories $\mathrm{Sp}_{X}^{\Sigma}, \mathrm{Sp}_{\text {const }}^{\Sigma} K$, and $\mathrm{Sp}^{\Sigma}$. The commutative diagram

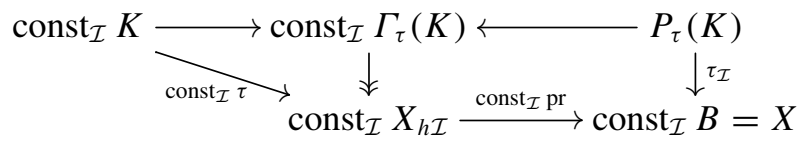

with pr: $X_{h \mathcal{I}}=B \times B \mathcal{I} \rightarrow B$ the projection then provides the desired isomorphisms

$$
(E, X)_{n}(K, \tau) \cong E_{n}(K, \operatorname{pr} \circ \tau) \quad \text { and } \quad(E, X)^{n}(K, \tau) \cong E^{n}(K, \operatorname{pr} \circ \tau) .
$$

Let $(R, M)$ be a parametrized ring spectrum in $\operatorname{Sp}_{\mathcal{R}}^{\Sigma}$ with multiplication on base $\mathcal{I}$-spaces $\mu: M \otimes M \rightarrow M$. Our next aim is to define pairings

$$
\begin{aligned}
& \times:(R, M)_{n}(K, \tau) \otimes(R, M)_{m}(L, \sigma) \longrightarrow(R, M)_{n+m}\left(K \times L, \tau \times \times_{\mu} \sigma\right) \\
& \times:(R, M)^{n}(K, \tau) \otimes(R, M)^{m}(L, \sigma) \longrightarrow(R, M)^{n+m}\left(K \times L, \tau \times{ }_{\mu} \sigma\right),
\end{aligned}
$$

where $\tau \times{ }_{\mu} \sigma$ refers to the composite $K \times L \stackrel{\tau \times \sigma}{\longrightarrow} M_{h \mathcal{I}} \times M_{h \mathcal{I}} \stackrel{\mu_{h \mathcal{I}}}{\longrightarrow} M_{h \mathcal{I}}$ in which the second map is the multiplication of the monoid $M_{h \mathcal{I}}$ in spaces. It follows from Remarks 6.18 and 6.21 that we may assume, without loss of generality, that $(R, M)$ is fibrant. Furthermore, if $(R, M)$ is commutative, we may assume that it is fibrant as a commutative parametrized ring spectrum by Theorem 6.17 and Remark 6.21. 
CONSTRUCTION 7.14. We observe that there is a natural chain of maps

$$
\begin{aligned}
\tau_{\mathcal{I}}^{*}(R, M) \bar{\wedge} \sigma_{\mathcal{I}}^{*}(R, M) & \rightarrow\left(\tau_{\mathcal{I}} \otimes \sigma_{\mathcal{I}}\right)^{*}((R, M) \bar{\wedge}(R, M)) \\
& \rightarrow\left(\tau_{\mathcal{I}} \otimes \sigma_{\mathcal{I}}\right)^{*} \mu^{*}(R, M) \rightarrow\left(\tau \times{ }_{\mu} \sigma\right)^{*}(R, M)
\end{aligned}
$$

in $\mathrm{Sp}_{\mathcal{R}}^{\Sigma}$ where the first map is an instance of (4.7), the second map is the canonical map induced by $\mu$, and the last map is induced by the monoidal structure map of the $\mathcal{I}$-spacification (see [45, Proposition 4.17] or [6, Lemma 4.5]). Precomposing this chain with cofibrant replacements of the $\bar{\wedge}$-factors in the source and using that $(R, M)$ is assumed to be fibrant gives a map

$$
\mathbb{R} \tau_{\mathcal{I}}^{*}(R, M) \bar{\wedge}_{\mathbb{L}} \mathbb{R} \sigma_{\mathcal{I}}^{*}(R, M) \rightarrow \mathbb{R}\left(\tau \times_{\mu} \sigma\right)_{\mathcal{I}}^{*}(R, M)
$$

on the homotopy category level. Precomposing it with the lax monoidal structure of $\mathbb{L} \Theta$ resulting from Lemma 4.6 and passing to homotopy groups induces the first pairing in (7.13). Using the monoidal structure map for $\mathbb{R} \Gamma$ resulting from (7.5) instead of that for $\mathbb{L} \Theta$ provides the analogous pairing in cohomology. Independence from the choices made during the construction, associativity and the fact that the unit of $(R, M)$ gives the unit $1 \in E_{0}(*, u)$ for the above product are now readily checked.

REMARK 7.16. Proposition 10.19 compares these pairings with the $\infty$ categorical variants from $[1,2]$.

Now we assume, in addition, that $(R, M)$ is commutative and check that these products are graded commutative. In order to give meaning to this, we first define an explicit twist homomorphism

$$
\text { tw: }(R, M)_{*}\left(K \times L, \tau \times \times_{\mu} \sigma\right) \rightarrow(R, M)_{*}\left(L \times K, \sigma \times_{\mu} \tau\right) .
$$

Since $M$ is supposed to be commutative, $M_{h \mathcal{I}}$ inherits the structure of an $E_{\infty}$ space with a canonical action of the Barratt-Eccles operad. Hence, there is an essentially unique homotopy $M_{h \mathcal{I}} \times M_{h \mathcal{I}} \times I \rightarrow M_{h \mathcal{I}}$ starting at the multiplication $\mu_{h \mathcal{I}}$ and ending at $\mu_{h \mathcal{I}} \circ \mathrm{tw}$. After precomposing with $\tau \times \sigma$, we get a homotopy $H$ from $\tau \times{ }_{\mu} \sigma$ to $\sigma \times{ }_{\mu} \tau \circ$ tw. Now we pull back $(R, M)$ via the $\mathcal{I}$-spacification of $H$ to obtain a chain of local equivalences

$$
\left(\tau \times_{\mu} \sigma\right)_{\mathcal{I}}^{*}(R, M) \stackrel{i_{0}}{\rightarrow} H_{\mathcal{I}}^{*}(R, M) \stackrel{i_{1}}{\leftarrow}\left(\sigma \times_{\mu} \tau \circ \mathrm{tw}\right)_{\mathcal{I}}^{*}(R, M) \stackrel{\mathrm{tw}}{\rightarrow}\left(\sigma \times_{\mu} \tau\right)_{\mathcal{I}}^{*}(R, M)
$$

in which $i_{0}$ and $i_{1}$ denote the endpoint inclusions. Applying $\mathbb{L} \Theta$, we get a diagram of stable equivalences, and (7.17) is the induced map of homotopy groups. Clearly, the latter does not depend on the choice of $H$. 
Proposition 7.19. The square

$$
\begin{aligned}
(R, M)_{n}(K, \tau) \otimes(R, M)_{m}(L, \sigma) \stackrel{\times}{\longrightarrow}(R, M)_{n+m}\left(K \times L, \tau \times \times_{\mu} \sigma\right) & \text { tw } \downarrow \\
& \stackrel{\text { tw }}{\downarrow} \\
(R, M)_{m}(L, \sigma) & \otimes(R, M)_{n}(K, \tau) \stackrel{\times}{\longrightarrow}(R, M)_{m+n}\left(L \times K, \sigma \times_{\mu} \tau\right)
\end{aligned}
$$

commutes up to the sign $(-1)^{n m}$. An analogous statement holds for parametrized cohomology groups.

Proof. It suffices to consider the topological setting. Let us write $\tau_{\mathcal{I}} \nabla_{\mu} \sigma_{\mathcal{I}}$ for the composition of $\tau_{\mathcal{I}} \otimes \sigma_{\mathcal{I}}$ with the multiplication $\mu: M \otimes M \rightarrow M$. The commutativity assumption on $(R, M)$ implies that the first square in the diagram

$$
\begin{aligned}
\tau_{\mathcal{I}}^{*}(R, M) & \bar{\wedge} \sigma_{\mathcal{I}}^{*}(R, M) \longrightarrow\left(\tau_{\mathcal{I}} \otimes_{\mu} \sigma_{\mathcal{I}}\right)^{*}(R, M) \longrightarrow\left(\tau \times_{\mu} \sigma\right)_{\mathcal{I}}^{*}(R, M) \\
{ }^{\mathrm{tw}} \downarrow & \downarrow \\
\sigma_{\mathcal{I}}^{*}(R, M) & \stackrel{\mathrm{tw}}{\wedge} \tau_{\mathcal{I}}^{*}(R, M) \longrightarrow\left(\sigma_{\mathcal{I}} \otimes_{\mu} \tau_{\mathcal{I}}\right)^{*}(R, M) \longrightarrow\left(\sigma \times_{\mu} \tau\right)_{\mathcal{I}}^{*}(R, M)
\end{aligned}
$$

is commutative. Here the horizontal maps are defined as in (7.15). It follows from the proof of [45, Lemma 6.7] that the maps $\pi: \bar{M} \rightarrow \operatorname{const}_{\mathcal{I}} M_{h \mathcal{I}}$ and $t: \bar{M} \rightarrow M$ going into the definition of the $\mathcal{I}$-spacification functor are compatible with the actions of the Barratt-Eccles operad on these $\mathcal{I}$-spaces. Hence, there is a commutative diagram of homotopies

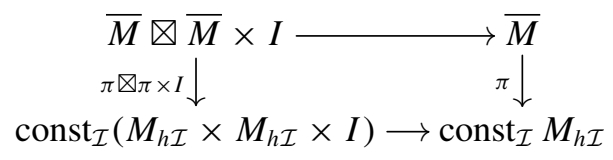

where the bottom homotopy is the one used to define the homotopy $H$ in (7.18) and the upper homotopy starts at $\bar{\mu}$ and ends at $\bar{\mu} \circ \mathrm{tw}$. Furthermore, the composition of the upper homotopy with $t$ is the constant homotopy on $t \circ \bar{\mu}$. Using both of these homotopies, we get a natural map of $\mathcal{I}$-spaces

$$
P_{\tau}(K) \otimes P_{\sigma}(L) \times I \rightarrow P_{H}(K \times L \times I),
$$

where the notation $P_{\tau}(K)$ denotes the domains for the $\mathcal{I}$-spacifications as in (7.7). This is, in fact, a map of $\mathcal{I}$-spaces over $M$ when we augment the left-hand side via the constant homotopy on $\tau_{\mathcal{I}} \bigotimes_{\mu} \sigma_{\mathcal{I}}$. Pulling back $(R, M)$ along these augmentations, we end up with the commutative diagram 


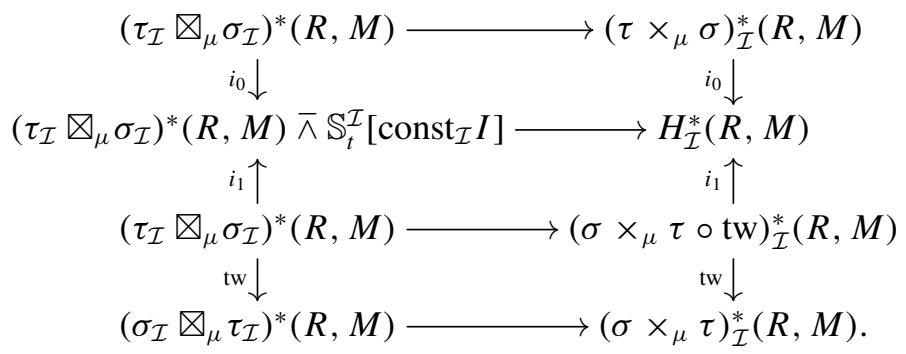

Applying $\mathbb{L} \Theta$ and identifying $\Theta\left(\mathbb{S}_{t}^{\mathcal{I}}\left[\right.\right.$ const $\left.\left._{\mathcal{I}} I\right]\right)$ with $\mathbb{S} \wedge I_{+}$, we get a homotopy commutative diagram from which we deduce the statement in the proposition. The cohomological statement follows by applying $\mathbb{R} \Gamma$ instead of $\mathbb{L} \Theta$.

7.20. Derived restriction as a left adjoint. We now begin to prepare for the proofs of Propositions 7.1 and 7.3. These proofs will rely on the following three lemmas which require us to work over simplicial sets and do not have direct topological counterparts. This will not lead to limitations for the propositions since they make statements about the homotopy category.

LEMMA 7.21. If $f: L \rightarrow K$ is a Kan fibration in $\mathrm{sSet}$, then the restriction functor $f^{*}: \operatorname{sSet}_{\mathcal{R}} / \iota_{b}(K) \rightarrow \operatorname{sSet}_{\mathcal{R}} / \iota_{b}(L)$ preserves weak equivalences and is left Quillen.

Proof. Since we are working over simplicial sets, $f^{*}$ has a right adjoint by the corresponding statement for the category of sets. Since base change preserves colimits and monomorphisms of sets, $f^{*}$ preserves cofibrations and colimits of simplicial sets and hence cofibrations in $\mathrm{sSet}_{\mathcal{R}}$ by their definition. Since sSet is right proper, base change along the Kan fibration $f$ preserves weak equivalences. Thus, $f^{*}$ is left Quillen.

LEMMA 7.22. The functor $\left(\text { const }_{\mathcal{I}} f\right)^{*}: \mathrm{Sp}_{\mathcal{R}}^{\Sigma} / \mathbb{S}_{b}^{\mathcal{I}}\left[\operatorname{const}_{\mathcal{I}} K\right] \rightarrow \mathrm{Sp}_{\mathcal{R}}^{\Sigma} / \mathbb{S}_{b}^{\mathcal{I}}\left[\right.$ const $\left._{\mathcal{I}} L\right]$ is left Quillen with respect to the absolute local model structures provided that $f: L \rightarrow K$ is a Kan fibration of Kan complexes.

Proof. The functor $\left(\text { const }_{\mathcal{I}} f\right)^{*}$ is a left adjoint by the corresponding statement for $\mathcal{S}_{\mathcal{R}}$ established in Lemma 7.21. Let $\mathbf{m}$, $\mathbf{n}$ be objects of $\mathcal{I}$, let $(W, P) \rightarrow \iota_{b}(K)$ be a map in $\mathcal{S}_{\mathcal{R}}$, and let $F_{\mathbf{m}}^{\mathrm{Sp}_{\mathcal{R}}^{\Sigma}}(W, P) \rightarrow \mathbb{S}_{b}^{\mathcal{I}}\left[\operatorname{const}_{\mathcal{I}} K\right]$ be the resulting object in $\mathrm{Sp}_{\mathcal{R}}^{\Sigma} / \mathbb{S}_{b}^{\mathcal{I}}\left[\right.$ const $\left._{\mathcal{I}} K\right]$. Then there is a natural isomorphism 


$$
\begin{array}{r}
\left(\left(\text { const }_{\mathcal{I}} f\right)^{*} F_{\mathbf{m}}^{\mathrm{Sp}_{\mathcal{R}}^{\Sigma}}(W, P)\right)(\mathbf{n})=\left(\operatorname{const}_{\mathcal{I}} f\right)^{*}\left(\coprod_{\alpha \in \mathcal{I}(\mathbf{m}, \mathbf{n})}(W, P) \bar{\wedge} S^{\mathbf{n}-\alpha}\right) \\
\stackrel{\cong}{\rightarrow} \coprod_{\alpha \in \mathcal{I}(\mathbf{m}, \mathbf{n})} f^{*}(W, P) \bar{\wedge} S^{\mathbf{n}-\alpha}=F_{\mathbf{m}}^{\mathrm{Sp}_{\mathcal{R}}^{\Sigma}}\left(f^{*}(W, P)\right)(\mathbf{n})
\end{array}
$$

where the coproducts are taken in $\mathcal{S}_{\mathcal{R}}$ and the base change on the right-hand side is formed along $(W, P) \rightarrow \iota_{b}(K)$. Since the cofibrations and generating acyclic cofibrations of the absolute level model structure on $\mathrm{Sp}_{\mathcal{R}}^{\Sigma} / \mathbb{S}_{b}^{\mathcal{I}}$ [const $\left.{ }_{\mathcal{I}} K\right]$ are obtained from those of $\mathrm{Sp}_{\mathcal{R}}^{\Sigma}$ by allowing all possible augmentations [24], the claim for the level model structure follows from the isomorphism (7.23) and Lemma 7.21. Since we assume $K$ to be fibrant, $\mathbb{S}_{b}^{\mathcal{I}}$ [const ${ }_{\mathcal{I}} K$ ] is fibrant in $\mathrm{Sp}_{\mathcal{R}}^{\Sigma}$ so that we can use [46, Proposition 3.4] to deduce that the local model structure on $\mathrm{Sp}_{\mathcal{R}}^{\Sigma} / \mathbb{S}_{b}^{\mathcal{I}}\left[\right.$ const $\left._{\mathcal{I}} K\right]$ can be viewed as the left Bousfield localization at a set of maps whose domains and codomains are of the form $F_{\mathbf{m}}^{\mathrm{Sp}_{\mathcal{R}}^{\Sigma}}(W, P) \rightarrow \mathbb{S}_{b}^{\mathcal{I}}$ [const $\left.{ }_{\mathcal{I}} K\right]$. So (7.23) implies that $\left(\operatorname{const}_{\mathcal{I}} f\right)^{*}$ is also left Quillen with respect to the local model structure.

REMARK 7.24. The preceding lemma does not hold in general if we consider the base change along $\mathbb{S}_{b}^{\mathcal{I}}[g]$ for an arbitrary map of $\mathcal{I}$-spaces $g$ since in this case, the different levels of $g^{*} F_{\mathbf{m}}^{\mathrm{Sp}_{\mathcal{R}}^{\Sigma}}(W, P)$ are coproducts over $g(\mathbf{m})^{*}(W, P)$ which may vary in $\mathbf{m}$.

Recall that if $K$ is a space, $\operatorname{Sp}_{K}^{\Sigma}=\operatorname{Sp}_{\text {const }_{\mathcal{I}} K}^{\Sigma}$ is the stabilization of $\mathcal{S}_{K}$.

LEMMA 7.25. The functor (const $\mathcal{I} f)^{*}: \mathrm{Sp}_{K}^{\Sigma} \rightarrow \mathrm{Sp}_{L}^{\Sigma}$ is left Quillen if $f: L \rightarrow K$ is a fibration of Kan complexes.

Proof. The functor $\left(\operatorname{const}_{\mathcal{I}} f\right)^{*}$ is a left adjoint since we are working with simplicial sets. The homotopical statement follows from Lemma 7.22 and Corollary 6.8 (or by adapting the argument in Lemma 7.22 to $\operatorname{Sp}_{K}^{\Sigma}$ and $\mathrm{Sp}_{L}^{\Sigma}$ ).

We have now developed enough tools to verify the statements about $f^{*}: \mathrm{Sp}_{X}^{\Sigma} \rightarrow \mathrm{Sp}_{Y}^{\Sigma}$ and its monoidal behavior made in the beginning of this section.

Proof of Proposition 7.1. Using Proposition 6.15, it suffices to verify the claim in the simplicial case. Since $X$ is $\mathcal{I}$-equivalent to $X^{\prime}=\operatorname{const}_{\mathcal{I}}\left(X^{\mathrm{fib}}(\mathbf{0})\right)$, the Quillen equivalences relating $\mathrm{Sp}_{X}^{\Sigma}$ to $\mathrm{Sp}_{X^{\prime}}^{\Sigma}$ allow us to assume that $f$ is of the form const ${ }_{\mathcal{I}} g$ for a map of Kan complexes $g: L \rightarrow K$. We factor $g$ as an acyclic cofibration $k: L \rightarrow P$ followed by a fibration $h: P \rightarrow X$. Then $\left(\text { const }_{\mathcal{I}} h\right)^{*}$ is left Quillen 
by Lemma 7.22, and applying $\left(\text { const }_{\mathcal{I}} h\right)^{*}$ to objects that are both cofibrant and fibrant shows that $\mathbb{L}\left(\text { const }_{\mathcal{I}} h\right)^{*}=\mathbb{R}\left(\text { const }_{\mathcal{I}} h\right)^{*}$. Since $\left(\text { const }_{\mathcal{I}} k\right)^{*}$ participates in a Quillen equivalence by Lemma $5.17, \mathbb{R}\left(\operatorname{const}_{\mathcal{I}} k\right)^{*}$ is an equivalence of categories. Hence,

$$
\mathbb{R}\left(\text { const }_{\mathcal{I}} g\right)^{*}=\mathbb{R}\left(\text { const }_{\mathcal{I}} k\right)^{*} \circ \mathbb{R}\left(\text { const }_{\mathcal{I}} h\right)^{*}=\mathbb{R}\left(\text { const }_{\mathcal{I}} k\right)^{*} \circ \mathbb{L}\left(\text { const }_{\mathcal{I}} h\right)^{*}
$$

is a left adjoint.

Given maps of cofibrant $\mathcal{I}$-spaces $f: X^{\prime} \rightarrow X$ and $g: Y^{\prime} \rightarrow Y$ as well as cofibrant and fibrant objects $(E, X)$ in $\operatorname{Sp}_{X}^{\Sigma}$ and $(F, Y)$ in $\operatorname{Sp}_{Y}^{\Sigma}$, there is a chain of maps

$$
\begin{aligned}
& f^{*}(E, X)^{\mathrm{cof}} \bar{\wedge} g^{*}(F, Y)^{\mathrm{cof}} \rightarrow f^{*}(E, X) \bar{\wedge} g^{*}(F, Y) \\
& \rightarrow(f \otimes g)^{*}((E, X) \bar{\wedge}(F, Y)) \rightarrow(f \otimes g)^{*}((E, X) \bar{\wedge}(F, Y))^{\mathrm{fib}}
\end{aligned}
$$

induced by cofibrant replacements in $\mathrm{Sp}_{X^{\prime}}^{\Sigma}$ and $\mathrm{Sp}_{Y^{\prime}}^{\Sigma}$, the map (4.7), and a fibrant replacement in $\mathrm{Sp}_{X \bowtie Y}^{\Sigma}$.

Proof of Proposition 7.3. For the statement of the proposition, it is sufficient to show that the map (7.26) is a local equivalence. Arguing in $\mathrm{Sp}_{\mathcal{R}}^{\Sigma}$ with arguments analogous to those in the proof of Proposition 7.1, we may assume that $f=$ const $_{\mathcal{I}} \tilde{f}$ and $g=$ const $_{\mathcal{I}} \tilde{g}$, where $\tilde{f}: K^{\prime} \rightarrow K$ and $\tilde{g}: L^{\prime} \rightarrow L$ are Kan fibrations of Kan complexes. Then $f \otimes g \cong \operatorname{const}_{\mathcal{I}}(\tilde{f} \times \tilde{g})$, and it follows from Lemma 7.25 that the first and the last map in (7.26) are local equivalences. Working over a constant base, Lemma 2.29 and the fact that base change preserves pullbacks show that the map (4.7) is even an isomorphism.

We conclude with an explicit description of the topological version of $\mathbb{R} \Gamma=$ $\mathbb{R}(Y \rightarrow *)_{*}$ and its monoidal structure in an important special case. This will become relevant in [21].

By [32, Proposition 1.5], the functor $f^{*}: \mathrm{Sp}_{\text {const }_{\mathcal{I}}}^{\Sigma} \rightarrow \mathrm{Sp}_{\text {const }_{\mathcal{I}} L}^{\Sigma}$ admits a right adjoint if and only if the map $f: L \rightarrow K$ is open. This is certainly the case for the map $r: K \rightarrow *$ and the adjoint $\Gamma$ is given by sending a parametrized spectrum $\left(E\right.$, const $\left._{\mathcal{I}} K\right)$ to the spectrum whose $m$ th level is given by the section space of the projection $E(\mathbf{m}) \rightarrow K$.

LEMMA 7.27. Suppose that $K$ is a cell complex or more generally cofibrant. Then $\Gamma: \mathrm{Sp}_{\text {const } \mathcal{I}_{K}}^{\Sigma} \rightarrow \mathrm{Sp}^{\Sigma}$ preserves (positive) level equivalences between parametrized spectra whose projections are Serre fibrations. In particular, $\Gamma$ preserves local equivalences between (positive) locally fibrant spectra and carries these to (positive) fibrant spectra. 
Proof. This is immediate from the fiber sequence $\Gamma(B, E) \rightarrow E^{B} \rightarrow B^{B}$, whenever $E \rightarrow B$ is a Serre fibration and $B$ is a cell complex, and the fact that $(-)^{B}$ preserves weak equivalences. The last claim follows since $\Gamma$ commutes with taking (fiberwise) loops by adjunction.

Thus, $\mathbb{R} \Gamma\left(E\right.$, const $\left._{\mathcal{I}} K\right)$ is represented by $\Gamma\left(E^{\mathrm{fib}}\right.$, const $\left._{\mathcal{I}} K\right)$, the value of $\Gamma$ on a locally fibrant replacement of $E$. Furthermore, by construction, the map

$$
\mathbb{R} \Gamma\left(E, \text { const }_{\mathcal{I}} K\right) \wedge_{\mathbb{L}} \mathbb{R} \Gamma\left(F, \text { const }_{\mathcal{I}} L\right) \rightarrow \mathbb{R} \Gamma\left(E \bar{\wedge}_{\mathbb{L}} F, \text { const }_{\mathcal{I}} K \times L\right)
$$

is represented by the natural map

$$
\Gamma\left(E, \text { const }_{\mathcal{I}} K\right) \wedge \Gamma\left(F, \text { const }_{\mathcal{I}} L\right) \longrightarrow \Gamma\left(E \bar{\wedge} F, \text { const }_{\mathcal{I}} K \times L\right)
$$

taking products of sections, whenever $\left(E\right.$, const $\left._{\mathcal{I}} K\right)$ and $\left(F, \operatorname{const}_{\mathcal{I}} L\right)$ are bifibrant.

A similar description still applies when we are presented with a (positive) level equivalence $f: X \rightarrow$ const $_{\mathcal{I}} K$. For $(E, X) \in \operatorname{Sp}_{X}^{\Sigma}$, we then find

$$
\mathbb{R} \Gamma(E, X) \cong \mathbb{R} \Gamma \mathbb{R} f_{*}(E, X) \cong \Gamma\left(\mathbb{L} f_{!}(E, X)\right)^{\mathrm{fib}},
$$

an observation which is made use of in the comparison of operator algebraic and homotopical twisted $K$-theory in [21, Proposition 6.2].

\section{The universal line bundle}

In this section, we construct an important example of a commutative parametrized ring spectrum, namely the universal line bundle $\gamma_{R}$ associated with a commutative symmetric ring spectrum $R$. We are interested in $\gamma_{R}$ for several reasons. In Section 10.12, we show that it represents its $\infty$-categorical counterpart studied in $[1,2]$. This leads to a multiplicative comparison of the parametrized (co)homology groups from Section 7 with the $\infty$-categorical ones from [1]. The universal line bundle also allows us to relate multiplicative pointset-level Thom spectrum functors to $\infty$-categorical ones (see Theorem 1.8). Finally, it also plays a prominent role in the multiplicative comparison of twisted $K$-theory spectra in [21].

8.1. The construction of the universal line bundle. In the following, we use the notion of commutative $\mathcal{I}$-space monoids from Definition 4.1, the positive $\mathcal{I}$-model structure on the resulting category of commutative $\mathcal{I}$-space monoids $\mathcal{C S}^{\mathcal{I}}$ [42, Section 3], and the adjunction $\mathbb{S}^{\mathcal{I}}: \mathcal{C} \mathcal{S}^{\mathcal{I}} \rightleftarrows \mathcal{C} \operatorname{Sp}^{\Sigma}: \Omega^{\mathcal{I}}$ relating them 
to commutative symmetric ring spectra [42, (3.9)]. Moreover, we say that a commutative $\mathcal{I}$-space monoid $M$ is grouplike if the monoid $\pi_{0}\left(M_{h \mathcal{I}}\right)$ is a group [42, Section 3.17].

Let $R$ be a positive fibrant commutative symmetric ring spectrum. Its multiplicative $E_{\infty}$ space is modeled by the commutative $\mathcal{I}$-space monoid $\Omega^{\mathcal{I}}(R)$, and its units $\mathrm{GL}_{1}^{\mathcal{I}} R$ are given by the subcommutative $\mathcal{I}$-space monoid of invertible path components of $\Omega^{\mathcal{I}}(R)$. The fibrancy condition on $R$ is needed to ensure that $\Omega^{\mathcal{I}}(R)$ and $\mathrm{GL}_{1}^{\mathcal{I}} R$ capture a well-defined homotopy type. It can be enforced by applying a fibrant replacement to $R$ (and could be relaxed to only asking $R$ to be positive level fibrant and semistable [6, Remark 2.6]).

We let $G=\left(\mathrm{GL}_{1}^{\mathcal{I}} R\right)^{\text {cof }}$ be a cofibrant replacement in the positive $\mathcal{I}$-model structure on $\mathcal{C S}^{\mathcal{I}}$. The adjoint of $G \rightarrow \mathrm{GL}_{1}^{\mathcal{I}} R \rightarrow \Omega^{\mathcal{I}}(R)$ is a map of commutative symmetric ring spectra $\mathbb{S}^{\mathcal{I}}[G] \rightarrow R$. Via the strong symmetric monoidal functor $\mathbb{S}_{t}^{\mathcal{I}}$ from Lemma 6.22, $G$ also gives rise to a commutative monoid $\mathbb{S}_{t}^{\mathcal{I}}[G]=$ $\mathbb{S}_{\mathcal{R}}^{\mathcal{I}}\left[\iota_{t}(G)\right]=\mathbb{S}_{\mathcal{R}}^{\mathcal{I}}[G \amalg G, G]$ in $\operatorname{Sp}_{\mathcal{R}}^{\Sigma}$ whose base commutative $\mathcal{I}$-space monoid is $G$. The unique map $G \rightarrow *$ induces a commutative monoid map $(G \amalg G, G)$ $\rightarrow(* \amalg G, *)$ in $\mathcal{S}_{\mathcal{R}}^{\mathcal{I}}$, and the composite

$$
\mathbb{S}_{t}^{\mathcal{I}}[G] \rightarrow \mathbb{S}_{\mathcal{R}}^{\mathcal{I}}[* \amalg G, *] \stackrel{\cong}{\rightarrow} \mathbb{S}^{\mathcal{I}}[G] \rightarrow R
$$

allows us to view $R$ as a commutative $\mathbb{S}_{t}^{\mathcal{I}}[G]$-algebra in $\mathrm{Sp}_{\mathcal{R}}^{\Sigma}$. We may also view $\mathbb{S}=\mathbb{S}_{t}^{\mathcal{I}}[*]$ as a commutative $\mathbb{S}_{t}^{\mathcal{I}}[G]$-algebra via the map induced by $G \rightarrow *$. Altogether, this allows us to form the two-sided bar construction

$$
B^{\bar{\wedge}}\left(\mathbb{S}, \mathbb{S}_{t}^{\mathcal{I}}[G], R\right)=\left|[q] \mapsto \mathbb{S} \bar{\wedge} \mathbb{S}_{t}^{\mathcal{I}}[G]^{\bar{\wedge} q} \bar{\wedge} R\right| .
$$

Being the realization of a simplicial object in $\mathcal{C} \operatorname{Sp}_{\mathcal{R}}^{\Sigma}$, it is itself a commutative parametrized ring spectrum. Its underlying commutative $\mathcal{I}$-space monoid is $B G=B(*, G, *)$, the bar construction of $G$ with respect to $\bigotimes$. As explained in [6, Section 2.9], $B G$ classifies $G$-modules. Its underlying $E_{\infty}$ space $(B G)_{h \mathcal{I}} \simeq$ $B\left(G_{h \mathcal{I}}\right)$ models the usual classifying space $B \mathrm{GL}_{1} R$ of the units of $R$.

DEFINITION 8.2. Let $R$ be a positive fibrant commutative symmetric ring spectrum in simplicial sets. Its universal line bundle is defined to be

$$
\gamma_{R}=\left(B^{\bar{\lambda}}\left(\mathbb{S}, \mathbb{S}_{t}^{\mathcal{I}}[G], R\right)\right)^{\mathrm{fib}},
$$

a fibrant replacement of $B^{\bar{\wedge}}\left(\mathbb{S}, \mathbb{S}_{t}^{\mathcal{I}}[G], R\right)$ in the positive local model structure on $\mathcal{C S p}{ }_{B G}^{\Sigma}$.

It follows from Lemmas 8.7 and 8.8 and the fact that $G$ is flat as an $\mathcal{I}$ space [42, Proposition 3.15(i)] that a stable equivalence $R \rightarrow R^{\prime}$ of positive fibrant commutative symmetric ring spectra induces a local equivalence $\gamma_{R} \rightarrow \gamma_{R}^{\prime}$. 
REMARK 8.3. The above construction can also be carried out for notnecessarily commutative ring spectra $R$ by using associative cofibrant and fibrant replacements instead of commutative ones. In this case, $\gamma_{R}$ is only an $R$-module and no longer a parametrized ring spectrum. The constructions from Section 7.9 still produce twisted $R$-(co)homology functors, but these are no longer equipped with products.

REMARK 8.4. For a positive fibrant commutative symmetric ring spectrum in topological spaces, we cannot directly implement Definition 8.2 because we have not established the topological version of the model structure on $\mathcal{C} \operatorname{Sp}_{B G}^{\Sigma}$ (and the topological counterparts of Lemmas 8.7 and 8.8). Rather than going through this, we content ourselves with the following construction: Given a positive fibrant commutative symmetric ring spectrum $R$ in topological spaces, we apply the above construction to Sing $R$ and define $\gamma_{R}$ to be $\left|\gamma_{\operatorname{Sing} R}\right|$. Then the realization of the simplicial $B G$ models the topological one by the discussion in Section 9.4, and $\left|\gamma_{\text {Sing } R}\right|$ is locally fibrant by Remark 6.21.

We again work over simplicial sets and let $E$ be an $R$-module spectrum. Then we can view $E$ as an $\mathbb{S}_{t}^{\mathcal{I}}[G]$-module by restriction along $\mathbb{S}_{t}^{\mathcal{I}}[G] \rightarrow R$ and generalize $\gamma_{R}$ by considering $\gamma_{E}=\left(B^{\bar{\wedge}}\left(\mathbb{S}, \mathbb{S}_{t}^{\mathcal{I}}[G], E\right)\right)^{\mathrm{fib}}$. Here the fibrant replacement is taken in a lifted model structure on $B^{\bar{\lambda}}\left(\mathbb{S}, \mathbb{S}_{t}^{\mathcal{I}}[G], R\right)$-module spectra that exists by [41, Proposition 3.4.2]. Based on this notion, we now describe the behavior of universal bundles under pullback. On the one hand, this is crucial for the applications in [21], and on the other, it shows that the fiber of $\gamma_{E}$ over the basepoint of $B G$ is just $E$ itself, as should be expected.

Proposition 8.5. We work in simplicial sets and let $H \rightarrow G$ be a map of $\mathcal{I}$ space monoids with $H$ flat and grouplike. Then the canonical map

$$
B^{\bar{\wedge}}\left(\mathbb{S}, \mathbb{S}_{t}^{\mathcal{I}}[H], E\right) \rightarrow(B H \rightarrow B G)^{*}\left(\gamma_{E}\right)
$$

is a local equivalence of parametrized spectra. When $E=R$ and $H$ is commutative, it is a local equivalence of commutative $B H$-relative parametrized ring spectra.

The proof requires some preparation and will be given at the end of this section.

8.6. Homotopy invariance properties. We now establish a series of lemmas needed for the homotopy invariance of $\gamma_{R}$, the proof of Proposition 8.5, and the next section. For this, we work again only over $\mathcal{I}$-spaces and (parametrized) symmetric spectra of simplicial sets. 
The realization of simplicial objects in $\mathrm{Sp}_{\mathcal{R}}^{\Sigma}$ can be defined by diagonalizing along the two simplicial directions and immediately lifts to a realization functor $\operatorname{Fun}\left(\Delta^{\mathrm{op}}, \mathcal{C} \operatorname{Sp}_{\mathcal{R}}^{\Sigma}\right) \rightarrow \mathcal{C} \operatorname{Sp}_{\mathcal{R}}^{\Sigma}$.

LEMMA 8.7. Let $\varphi:(E, X) \bullet \rightarrow(F, Y)$. be a natural transformation between simplicial objects in $\operatorname{Sp}_{\mathcal{R}}^{\Sigma}$ with each $(E, X)_{q} \rightarrow(F, Y)_{q}$ a local equivalence. Then the realization of $\varphi$ is a local equivalence.

Proof. We consider the Reedy model structure on Fun $\left(\Delta^{\mathrm{op}}, \mathrm{Sp}_{\mathcal{R}}^{\Sigma}\right)$ induced by the absolute local model structure. The realization of a Reedy cofibrant replacement is a level equivalence by applying the realization lemma for simplicial sets. The claim follows because realization preserves weak equivalences between Reedy cofibrant objects.

We say that an ordinary symmetric spectrum $E$ is flat if it is cofibrant in the flat (or $S$-) model structure on symmetric spectra (see [47, 49]). This notion is useful because $E \wedge-$ preserves stable equivalences if $E$ is flat and the underlying symmetric spectra of cofibrant objects in the positive stable model structure on $\mathcal{C S p}^{\Sigma}$ are flat. Analogously, there is the notion of a flat $\mathcal{I}$-space such that $X \otimes-$ preserves $\mathcal{I}$-equivalences if $X$ is flat and underlying $\mathcal{I}$-spaces of cofibrant commutative $\mathcal{I}$-space monoids are flat [42, Section 3.8].

LEMMA 8.8. Let $(E, X)$ be cofibrant in $\mathrm{Sp}_{\mathcal{R}}^{\Sigma}$, let $F$ be a flat symmetric spectrum, and let $Z$ be a flat $\mathcal{I}$-space. Then $(E, X) \bar{\Lambda}-, F \bar{\wedge}-$, and $\mathbb{S}_{t}^{\mathcal{I}}[Z] \bar{\wedge}-$ preserve local equivalences as functors $\mathrm{Sp}_{\mathcal{R}}^{\Sigma} \rightarrow \mathrm{Sp}_{\mathcal{R}}^{\Sigma}$.

Proof. By [41, Propositions 2.3.10 and 3.3.6], the category $\mathrm{Sp}_{\mathcal{R}}^{\Sigma}$ also has a flat absolute local model structure with more cofibrations and with weak equivalences the local equivalences. We call the cofibrant objects in this model structure flat and note that the $\bar{\wedge}$-product with flat objects preserves local equivalences by the flatness statement subsumed in [41, Proposition 3.4.2]. Hence, $(E, X) \bar{\wedge}-$ preserves local equivalences. One can check on the generating cofibrations that both $\mathbb{S}_{t}^{\mathcal{I}}: \mathcal{S}^{\mathcal{I}} \rightarrow \mathrm{Sp}_{\mathcal{R}}^{\Sigma}$ and the inclusion functor $\mathrm{Sp}^{\Sigma} \rightarrow \mathrm{Sp}_{\mathcal{R}}^{\Sigma}$ preserve the cofibrations of the flat model structures and thus flat objects.

COROLlary 8.9. If $(E, X)$ is an object in $\operatorname{Sp}_{\mathcal{R}}^{\Sigma}$ and $f: Y \rightarrow Y^{\prime}$ is an $\mathcal{I}$ equivalence between flat $\mathcal{I}$-spaces, then $\mathbb{S}_{t}^{\mathcal{I}}[f] \bar{\wedge}(E, X)$ is a local equivalence.

Proof. Taking a cofibrant replacement of $(E, X)$, this follows from the previous lemma by two out of three for local equivalences. 
Our next aim is to obtain homotopy invariance results for restriction functors beyond what can be deduced directly from Lemma 7.22.

LEMMA 8.10. If $f: Y \rightarrow X$ is a fibration between fibrant objects in the absolute $\mathcal{I}$-model structure on $\mathcal{S}^{\mathcal{I}}$, then $f^{*}: \mathrm{Sp}_{\mathcal{R}}^{\Sigma} / \mathbb{S}_{b}^{\mathcal{I}}[X] \rightarrow \mathrm{Sp}_{\mathcal{R}}^{\Sigma} / \mathbb{S}_{b}^{\mathcal{I}}[Y]$ preserves local equivalences.

Proof. Since absolute $\mathcal{I}$-fibrant $\mathcal{I}$-spaces are naturally level equivalent to constant $\mathcal{I}$-spaces, we may assume that $f$ is a fibration of fibrant and constant $\mathcal{I}$-spaces. Then $f^{*}$ is left Quillen by Lemma 7.22 and right Quillen by general model category theory. Hence, $f^{*}$ preserves weak equivalences.

We now consider the following commutative diagram in $\mathrm{Sp}_{\mathcal{R}}^{\Sigma}$ where the righthand horizontal maps are the identity on the base:

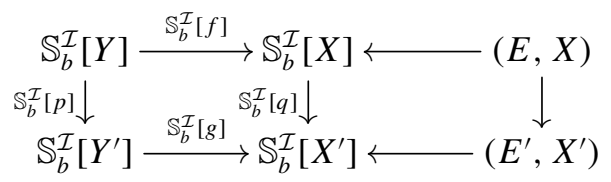

The next proposition uses the description of $f^{*}$ from (3.27) and essentially states that the local model structure satisfies a weak form of right properness where the fibrations are only allowed to be in the image of $\mathbb{S}_{b}^{\mathcal{I}}$. Its proof is based on Bousfield's observation that it is sufficient to check right properness of model categories on fibrations between fibrant objects [10, Lemma 9.4].

Proposition 8.11. If $f$ and $g$ are absolute $\mathcal{I}$-fibrations, $p$ and $q$ are $\mathcal{I}$ equivalences, and $(E, X) \rightarrow\left(E^{\prime}, X^{\prime}\right)$ is a local equivalence, then the induced map of pullbacks $f^{*}(E, X) \rightarrow g^{*}\left(E^{\prime}, X^{\prime}\right)$ is a local equivalence. The same statement holds when working over topological spaces.

Setting $p=\mathrm{id}$ and $q=\mathrm{id}$ in the proposition implies that the statements of Lemmas 7.22 and 8.10 hold without the fibrancy conditions on the objects.

Proof. Since the topological statement follows from the simplicial one by applying the singular complex, it suffices to verify the latter. By choosing a replacement of $f$ by an $\mathcal{I}$-fibration between $\mathcal{I}$-fibrant objects $\tilde{f}: \tilde{Y} \rightarrow \tilde{X}$, Lemma 3.19 provides the left-hand square in the following commutative diagram:

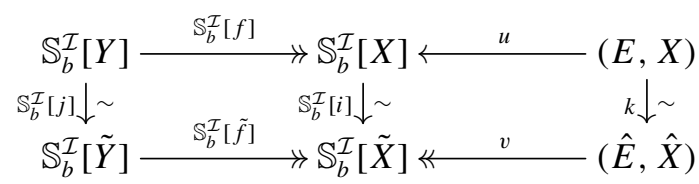


The right-hand square is obtained by factoring $(E, X) \rightarrow \mathbb{S}_{b}^{\mathcal{I}}[\tilde{X}]$ as a local equivalence followed by a fibration. We get the following sequence of maps where $(-)^{*}$ denotes the base change along the respective map in $\mathrm{Sp}_{\mathcal{R}}^{\Sigma}$ :

$\mathbb{S}_{b}^{\mathcal{I}}[f]^{*}(E, X) \cong u^{*}\left(\mathbb{S}_{b}^{\mathcal{I}}[Y]\right) \stackrel{\sim}{\rightarrow} u^{*}\left(\mathbb{S}_{b}^{\mathcal{I}}[i]^{*}\left(\mathbb{S}_{b}^{\mathcal{I}}[\tilde{Y}]\right)\right) \stackrel{\cong}{\rightarrow} \mathbb{S}_{b}^{\mathcal{I}}[\tilde{f}]^{*}(E, X) \stackrel{\sim}{\rightarrow}(\hat{E}, \hat{X})$.

Here the first map is a local equivalence since $u^{*}$ is right Quillen when viewed as a functor $\operatorname{Sp}_{R}^{\Sigma} / \mathbb{S}_{b}^{\mathcal{I}}[X] \rightarrow \operatorname{Sp}_{R}^{\Sigma} /(E, X)$ and $\mathbb{S}_{b}^{\mathcal{I}}[Y] \rightarrow \mathbb{S}_{b}^{\mathcal{I}}[i]^{*}\left(\mathbb{S}_{b}^{\mathcal{I}}[\tilde{Y}]\right)$ is a weak equivalence between fibrant objects in $\mathrm{Sp}_{R}^{\Sigma} / \mathbb{S}_{b}^{\mathcal{I}}[X]$ because the $\mathcal{I}$-model structure on $\mathcal{S}^{\mathcal{I}}$ is right proper. The last map is a local equivalence by Lemma 8.10. Hence, we have shown that the pullback of the top row is locally equivalent to the pullback of the bottom row, and the latter is homotopy invariant since both maps are fibrations with fibrant codomain. Since this construction can be arranged to be natural with respect to $(E, X) \rightarrow\left(E^{\prime}, X^{\prime}\right)$ and $Y \rightarrow Y^{\prime}$, the claim follows.

LEMMA 8.12. Let $f:(Z, Y) \rightarrow\left(Z^{\prime}, Y^{\prime}\right)$ be a map in $\mathcal{S}_{\mathcal{R}}^{\mathcal{I}}$ such that both $Z \rightarrow Z^{\prime}$ and $Y \rightarrow Y^{\prime}$ are absolute level (respectively $\mathcal{I}$-) equivalences in $\mathcal{S}^{\mathcal{I}}$. Then $\mathbb{S}_{\mathcal{R}}^{\mathcal{I}}[f]$ is an absolute level (respectively local) equivalence in $\mathrm{Sp}_{\mathcal{R}}^{\Sigma}$.

Proof. Since $-\bar{\wedge} S^{m}: \mathcal{S}_{\mathcal{R}} \rightarrow \mathcal{S}_{\mathcal{R}}$ preserves weak equivalences as we work over sSet, $\mathbb{S}_{\mathcal{R}}^{\mathcal{I}}$ preserves level equivalences. Since $\mathbb{S}_{\mathcal{R}}^{\mathcal{I}}$ is left Quillen with respect to the local model structures, arguing with a cofibrant replacement shows the second claims.

LEMMA 8.13. Let $f: Y \rightarrow X$ be an absolute $\mathcal{I}$-fibration in $\mathcal{S}^{\mathcal{I}}$, let $Z \rightarrow X$ be a map of $\mathcal{I}$-spaces, and let $E$ be a flat symmetric spectrum. Then the canonical map

$$
\mathbb{S}_{\mathcal{R}}^{\mathcal{I}}\left[Y \amalg f^{*}(Z), Y\right] \bar{\wedge} E \rightarrow f^{*}\left(\mathbb{S}_{\mathcal{R}}^{\mathcal{I}}[X \amalg Z, X] \bar{\wedge} E\right)
$$

is a local equivalence.

Proof. Arguing with the absolute $\mathcal{I}$-model structure on $\mathcal{S}^{\mathcal{I}}$ and the Quillen equivalence $\operatorname{colim}_{\mathcal{I}}: \mathcal{S}^{\mathcal{I}} \rightleftarrows \mathcal{S}$ : const $_{\mathcal{I}}$, we can construct a commutative diagram

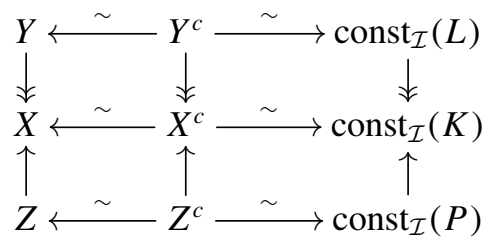

with the $\mathcal{I}$-fibrations and $\mathcal{I}$-equivalences as indicated. Arguing with this diagram, Lemmas 8.8 and 8.12 and Proposition 8.11 reduce the claim to the case where all 
$\mathcal{I}$-spaces are constant. In this situation, the map in question is an isomorphism by Lemma 2.29 and the explicit description of $\mathbb{S}_{\mathcal{R}}^{\mathcal{I}}$ in Construction 3.16.

Proof of Proposition 8.5. By Lemmas 8.7 and 8.8, both sides send cofibrant replacements of $R$ and $E$ to local equivalences. Thus, we may assume $E$ to be a cofibrant module over a cofibrant commutative ring spectrum and therefore to be flat as a symmetric spectrum. Next we choose a factorization of $f: B H \rightarrow B G$ into an acyclic cofibration $g: B H \rightarrow Y$ followed by a fibration $h: Y \rightarrow B G$ in the absolute $\mathcal{I}$-model structure and consider the following diagram:

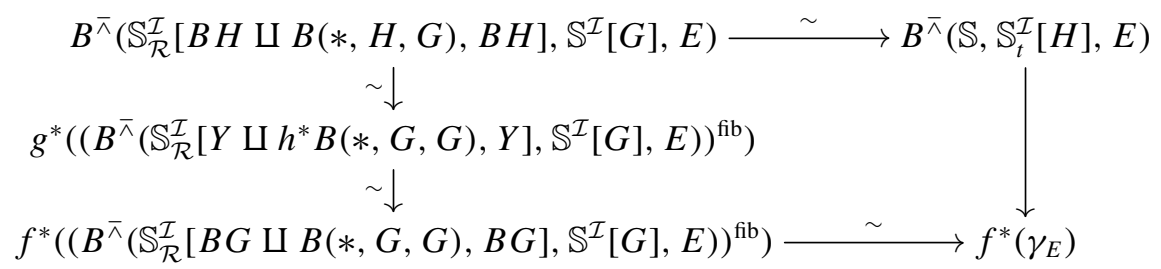

The top horizontal map arises by identifying $\mathbb{S}_{\mathcal{R}}^{\mathcal{I}}[B H \amalg B(*, H, G)]$ with the bar construction $B^{\bar{\lambda}}\left(\mathbb{S}, \mathbb{S}_{t}^{\mathcal{I}}[H], \mathbb{S}^{\mathcal{I}}[G]\right)$, commuting bar constructions, and using the map induced by the canonical stable equivalence $B\left(\mathbb{S}^{\mathcal{I}}[G], \mathbb{S}^{\mathcal{I}}[G], E\right) \rightarrow E$. The resulting map is a local equivalence since $B^{\bar{\lambda}}\left(\mathbb{S}, \mathbb{S}_{t}^{\mathcal{I}}[H],-\right)$ preserves local equivalences by Lemmas 8.7 and 8.8 . The lower horizontal map arises in the same way by setting $H=G$ and taking fibrant replacements and base change along the right Quillen functor $f^{*}$ in addition. The map

$$
\begin{aligned}
& B^{\bar{\lambda}}\left(\mathbb{S}_{\mathcal{R}}^{\mathcal{I}}\left[Y \amalg h^{*} B(*, G, G), Y\right], \mathbb{S}^{\mathcal{I}}[G], E\right) \\
& \quad \rightarrow B^{\wedge}\left(\mathbb{S}_{\mathcal{R}}^{\mathcal{I}}[B G \amalg B(*, G, G), B G], \mathbb{S}^{\mathcal{I}}[G], E\right)
\end{aligned}
$$

is a local equivalence by Lemmas 8.7 and 8.13. Since $h^{*}\left(\gamma_{E}\right)$ is fibrant, we can extend the resulting local equivalence to $h^{*}\left(\gamma_{E}\right)$ over a fibrant replacement of the domain and apply $g^{*}$ to get the lower left-hand vertical local equivalence. The upper left-hand vertical equivalence arises from the fact that $B(*, H, G)$ $\rightarrow h^{*} B(*, G, G)$ is an $\mathcal{I}$-equivalence since $G$ and $H$ are grouplike [6, Proof of Proposition 3.15], the homotopy invariance of $\mathbb{S}_{\mathcal{R}}^{\mathcal{I}}$ established in Lemma 8.12 and that of $B\left(-, \mathbb{S}^{\mathcal{I}}[G], E\right)$ resulting from Lemmas 8.7 and 8.8 and from Corollary 6.9. It follows that the right-hand vertical map is a local equivalence.

\section{Point-set-level Thom spectrum functors}

We now explain how our approach to parametrized spectra gives rise to a multiplicative $R$-module Thom spectrum functor. As an application, we compare it to various other approaches to generalized Thom spectra. 
9.1. Generalized Thom spectra via universal bundles. Let $R$ be a commutative ring spectrum in simplicial sets that is positive fibrant or, more generally, level fibrant and semistable (cf. [6, Remark 2.6]). We now write $\left(\mathrm{Sp}^{\Sigma}\right)_{R}$ for the category of (right) $R$-modules in $\mathrm{Sp}^{\Sigma}$ and $\left(\mathrm{Sp}_{\mathcal{R}}^{\Sigma}\right)_{R}$ for the category of (right) $R$-modules in $\operatorname{Sp}_{\mathcal{R}}^{\Sigma}$. Via the composite $R \rightarrow B^{\bar{\wedge}}\left(\mathbb{S}, \mathbb{S}_{t}^{\mathcal{I}}[G], R\right) \rightarrow \gamma_{R}$, we can view the universal line bundle $\gamma_{R}$ as a commutative $R$-algebra, that is, a commutative monoid with respect to the resulting product $\bar{\Lambda}_{R}$ in $\left(\operatorname{Sp}_{\mathcal{R}}^{\Sigma}\right)_{R}$. We obtain a Thom spectrum functor

$$
\begin{gathered}
T_{\mathcal{R}}^{\mathcal{I}}: \mathcal{S}^{\mathcal{I}} / B G \rightarrow\left(\operatorname{Sp}_{\mathcal{R}}^{\Sigma}\right)_{R} \rightarrow \operatorname{Mod}_{R}, \\
(f: X \rightarrow B G) \mapsto \Theta\left(f^{*} \gamma_{R}\right)=(X \rightarrow *) !\left(f^{*} \gamma_{R}\right) .
\end{gathered}
$$

This functor takes values in right $R$-modules since $\gamma_{R}$ is a right $R$-module and both base change and the collapse of base space functor $\Theta$ preserve right $R$ module structures as follows from the monoidality in Lemma 4.6 and (4.7). Precomposing $T_{\mathcal{R}}^{\mathcal{I}}$ with the $\mathcal{I}$-spacification $P_{B G}$ provides a space level Thom spectrum functor

$$
T_{\mathcal{R}}: \mathcal{S} /(B G)_{h \mathcal{I}} \rightarrow \operatorname{Mod}_{R}, \quad\left(\tau: K \rightarrow(B G)_{h \mathcal{I}}\right) \mapsto T_{\mathcal{R}}^{\mathcal{I}}\left(\tau_{\mathcal{I}}\right)
$$

that sends weak equivalences to stable equivalences and preserves actions of operads augmented over the Barratt-Eccles operad. Since $\gamma_{R}$ is fibrant and $\Theta$ coincides with its left derived functors, the homotopy groups $\pi_{n}\left(T_{\mathcal{R}}(\tau)\right)$ are just the parametrized homology groups associated with the universal line bundle $\gamma_{R}$ and the map $\tau$.

Proposition 9.3. The functor $T_{\mathcal{R}}^{\mathcal{I}}$ is lax symmetric monoidal and sends $\mathcal{I}$ equivalences over $B G$ to stable equivalences of $R$-modules. It preserves colimits, tensors with simplicial sets, and actions of operads in simplicial sets.

Proof. We get a natural map $f^{*}\left(\gamma_{R}\right) \bar{\wedge}_{R} g^{*}\left(\gamma_{R}\right) \rightarrow(f \otimes g)^{*}\left(\gamma_{R}\right)$ since $\gamma_{R}$ is a commutative $R$-algebra. This exhibits $\mathcal{S}^{\mathcal{I}} / B G \rightarrow\left(\operatorname{Sp}_{\mathcal{R}}^{\Sigma}\right)_{R}, f \mapsto f^{*}\left(\gamma_{R}\right)$ as a lax symmetric monoidal functor. Since $\Theta$ is strong symmetric monoidal by Lemma 4.6, it follows that $T_{\mathcal{R}}^{\mathcal{I}}$ is lax symmetric monoidal. For the homotopy invariance, we note that an $\mathcal{I}$-equivalence $g: Y \rightarrow X$ and a map $f: X \rightarrow B G$ give rise to a map $(f g)^{*}\left(\gamma_{R}\right)=g^{*} f^{*}\left(\gamma_{R}\right) \rightarrow f^{*}\left(\gamma_{R}\right)$ that is a local equivalence by the fibrancy assertion on $\gamma_{R}$ and Corollary 6.9. The functor $\Theta$ maps this local equivalence to a stable equivalence $T_{\mathcal{R}}^{\mathcal{I}}(f g) \rightarrow T_{\mathcal{R}}^{\mathcal{I}}(f)$. Compatibility with the tensor and colimits follows since the individual functors have this property.

If $R \rightarrow R^{\prime}$ is a stable equivalence between positive fibrant objects, we get a natural stable equivalence between the resulting Thom spectrum functors that is induced by the above local equivalence $\gamma_{R} \rightarrow \gamma_{R}^{\prime}$. 
9.4. Generalized Thom spectra via classifying spaces for $\boldsymbol{G}$-modules. We begin by reviewing the Thom spectrum functor introduced in [6]. In the latter paper, the focus is on the topological setting, but the analogous construction works equally well in the simplicial setting; cf. [6, Remark 3.7]. Thus, in the following discussion, the underlying category of spaces $\mathcal{S}$ can be either sSet or Top.

Let $R$ be a positive fibrant and flat commutative symmetric ring spectrum, write $\mathrm{GL}_{1}^{\mathcal{I}} R$ for its $\mathcal{I}$-space units, and let $G \rightarrow \mathrm{GL}_{1}^{\mathcal{I}} R$ be a cofibrant replacement in $\mathcal{C S}^{\mathcal{I}}$. We define $E G$ by choosing a factorization of the form

$$
B(*, G, G) \stackrel{\sim}{\longrightarrow} E G \longrightarrow B G
$$

in the positive model structure of $\mathcal{C} \mathcal{S}^{\mathcal{I}}$. Now let $U: \mathcal{S}^{\mathcal{I}} / B G \rightarrow \operatorname{Mod}_{G}$ be the functor to $G$-modules in $\mathcal{S}^{\mathcal{I}}$ sending a map $f: X \rightarrow B G$ to the pullback $U(f)$ of the diagram $X \stackrel{f}{\rightarrow} B G \leftarrow E G$ where both $X$ and $B G$ carry the trivial $G$ module structure. The fibrant replacement in (9.5) ensures that $U$ preserves $\mathcal{I}$ equivalences.

The $\mathcal{I}$-space version of the Thom spectrum functor [6, Definition 3.6] is the composite

$$
T_{E G}^{\mathcal{I}}: \mathcal{S}^{\mathcal{I}} / B G \stackrel{U}{\rightarrow} \operatorname{Mod}_{G} \stackrel{\mathbb{S}^{\mathcal{I}}}{\rightarrow} \operatorname{Mod}_{\mathbb{S}^{\mathcal{I}}[G]} \stackrel{B\left(-, \mathbb{S}^{\mathcal{I}}[G], R\right)}{\longrightarrow} \operatorname{Mod}_{R}
$$

where we use the subscript $E G$ to distinguish it from $T_{\mathcal{R}}^{\mathcal{I}}$. Precomposing $T_{E G}^{\mathcal{I}}$ with the $\mathcal{I}$-spacification (7.7) defines a space level Thom spectrum functor

$$
T_{E G}: \mathcal{S} / B G_{h \mathcal{I}} \rightarrow \operatorname{Mod}_{R}
$$

with favorable properties; see [6, Section 4.6]. It is proved in [6, Proposition 4.6] that $T_{E G}$ is homotopy invariant by our assumption that $R$ is flat.

REMARK 9.7. In [6], the commutative $\mathcal{I}$-space monoids $G$ and $E G$ were defined using the so-called flat $\mathcal{I}$-model structure on $\mathcal{C} \mathcal{S}^{\mathcal{I}}$. For the definition of the Thom spectrum functors $T_{E G}^{\mathcal{I}}$ and $T_{E G}^{\mathcal{I}}$, we may equally work well with the projective $\mathcal{I}$-model structure used in the present paper since the latter model structure has fewer cofibrations.

We now explain why the simplicial and topological versions are equivalent. First, geometric realization and singular complex induce Quillen equivalences between the simplicial and topological versions of commutative symmetric ring spectra and commutative $\mathcal{I}$-space monoids. Up to isomorphism, geometric realization commutes with $\mathbb{S}^{\mathcal{I}}$ and thus Sing commutes with $\Omega^{\mathcal{I}}$. Moreover, geometric realization preserves positive fibrant objects. When $R$ is a topological 
positive fibrant commutative symmetric ring spectrum, then $\operatorname{Sing}\left(\mathrm{GL}_{1}^{\mathcal{I}} R\right) \cong$ $\mathrm{GL}_{1}^{\mathcal{I}} \operatorname{Sing}(R)$. If $R$ is a positive fibrant commutative symmetric ring spectrum in simplicial sets and $G \rightarrow \mathrm{GL}_{1}^{\mathcal{I}} R$ is a cofibrant replacement of its units, then the adjoint of $\mathbb{S}^{\mathcal{I}}[|G|] \stackrel{\cong}{\rightarrow}\left|\mathbb{S}^{\mathcal{I}}[G]\right| \rightarrow|R|$ exhibits $|G|$ as a cofibrant replacement of $\mathrm{GL}_{1}^{\mathcal{I}}|R|$ since its image under Sing participates as the upper left-hand horizontal arrow in the commutative diagram

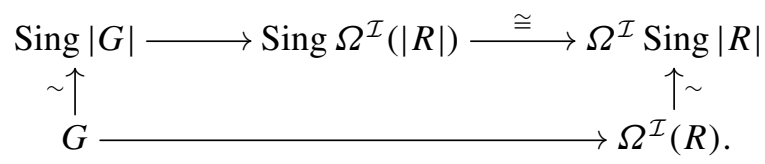

Hence, $|B G|$ models $B\left(\mathrm{GL}_{1}^{\mathcal{I}}|R|\right)^{\text {cof }}$. Since realization also preserves positive $\mathcal{I}$ fibrations, $|E G|$ models its topological counterpart for $|R|$.

Proposition 9.8. Let $R$ be a positive fibrant commutative symmetric ring spectrum in simplicial sets and let $f: X \rightarrow B G$ be a map of $\mathcal{I}$-spaces, also in simplicial sets. Defining the topological Thom spectrum functor $T_{|E G|}^{\mathcal{I}}$ for $|R|$ using $|G|$ and $|E G|$ as explained above, there is a natural isomorphism $\left|T_{E G}^{\mathcal{I}}(f)\right| \cong T_{|E G|}^{\mathcal{I}}(|f|)$. It induces a monoidal natural stable equivalence $\left.\left|T_{E G}(\tau)\right| \rightarrow T_{|E G|}|| \tau \mid\right)$ of space level Thom spectra preserving actions of operads augmented over the Barratt-Eccles operad.

Proof. The statement for $T_{E G}^{\mathcal{I}}$ follows since geometric realization preserves pullback and is strong symmetric monoidal both for $\mathcal{I}$-spaces and symmetric spectra. The space level version results from the natural $\mathcal{I}$-equivalence $\left|P_{B G}(\tau)\right| \rightarrow P_{|B G|}(|\tau|)$ induced by the adjunction $(|-|$, Sing).

Conversely, let $R$ be a topological positive fibrant commutative symmetric ring spectrum and $G \rightarrow \mathrm{GL}_{1}^{\mathcal{I}}(\operatorname{Sing} R)^{\text {cof }}$ be a cofibrant replacement of the units of a cofibrant replacement of $\operatorname{Sing} R$ in commutative ring spectra. Given any map $f: X \rightarrow|B G|$, a homotopy pullback construction provides a map $f^{\prime}: X^{\prime} \rightarrow B G$ such that $\left|X^{\prime}\right|$ and $X$ are weakly equivalent over $|B G|$ so that $\left|T_{E G}^{\mathcal{I}}\left(f^{\prime}\right)\right| \simeq T_{E G}^{\mathcal{I}}(f) \simeq T^{\mathcal{I}}(f)$ as modules over $\left|(\operatorname{Sing} R)^{\text {cof }}\right| \simeq R$. This shows that the topological Thom spectrum functor can be expressed in terms of the simplicial one.

9.9. Comparing $\boldsymbol{R}$-module Thom spectra. Our next aim is to compare the simplicial version of $T_{E G}^{\mathcal{I}}$ to the Thom spectrum functor $T_{\mathcal{R}}^{\mathcal{I}}$ of Section 9.1. For this, we consider the following commutative diagram in $\operatorname{Sp}_{B G}^{\Sigma}$ : 


$$
\begin{aligned}
& B^{\bar{\wedge}}\left(\mathbb{S}_{\mathcal{R}}^{\mathcal{I}}[B G \amalg B(*, G, G), B G], \mathbb{S}^{\mathcal{I}}[G], R\right) \stackrel{\sim}{\longrightarrow} B^{\bar{\wedge}}\left(\mathbb{S}_{\mathcal{R}}^{\mathcal{I}}[B G \amalg E G, B G], \mathbb{S}^{\mathcal{I}}[G], R\right) \\
& \sim \downarrow \quad \sim \downarrow v \\
& \mathbb{S}_{\mathcal{R}}^{\mathcal{I}}[B G \amalg B(*, G, G), B G] \bar{\wedge}_{\mathbb{S}^{\mathcal{I}}[G]} R \succ \sim \mathbb{S}_{\mathcal{R}}^{\mathcal{I}}[B G \amalg E G, B G] \bar{\wedge}_{\mathbb{S}^{\mathcal{I}}[G]} R
\end{aligned}
$$

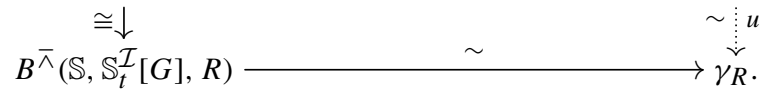

The two upper horizontal maps are induced by $B(*, G, G) \rightarrow E G$. The upper one is a local equivalence by Lemmas 8.7 and 8.12. To analyze the second, we again use the functor $\mathbb{S}_{\mathrm{ar}}^{\mathcal{I}}=\mathbb{S}_{\mathcal{R}}^{\mathcal{I}} \circ \iota_{\mathrm{ar}}: \operatorname{Ar}\left(\mathcal{S}^{\mathcal{I}}\right) \rightarrow \mathrm{Sp}_{\mathcal{R}}^{\Sigma}$ from (4.8). It induces a functor

$$
(G \rightarrow *) \downarrow \operatorname{Ar}\left(\mathcal{C S}^{\mathcal{I}}\right) \rightarrow \mathbb{S}^{\mathcal{I}}[G] \downarrow \mathcal{C} \operatorname{Sp}_{\mathcal{R}}^{\Sigma}
$$

from commutative $(G \rightarrow *)$-algebras in $\operatorname{Ar}\left(\mathcal{S}^{\mathcal{I}}\right)$ to commutative $\mathbb{S}^{\mathcal{I}}[G]$ algebras in $\operatorname{Sp}_{\mathcal{R}}^{\Sigma}$. With respect to the injective model structure on $\operatorname{Ar}\left(\mathcal{C S}^{\mathcal{I}}\right)$ (cf. Lemma 5.14), it sends the acyclic cofibration $B(*, G, G) \rightarrow E G$ over $B G$ to an acyclic cofibration of commutative $\mathbb{S}^{\mathcal{I}}[G]$-algebras in $\mathrm{Sp}_{\mathcal{R}}^{\Sigma}$. Extending the latter map along $\mathbb{S}^{\mathcal{I}}[G] \rightarrow R$ shows that the middle horizontal map in the diagram is an acyclic cofibration in $\mathcal{C} \operatorname{Sp}_{\mathcal{R}}^{\Sigma}$. The upper vertical maps are instances of the natural map from the two-sided bar construction to the relative $\bar{\wedge}$-product. The lower left-hand isomorphism results from commuting $\mathbb{S}_{\mathcal{R}}^{\mathcal{I}}$ with the bar construction (compare the argument in the proof of Proposition 8.5). The left-hand vertical composite can be identified with the map

$$
B^{\bar{\wedge}}\left(\mathbb{S}, \mathbb{S}_{t}^{\mathcal{I}}[G], B^{\bar{\wedge}}\left(\mathbb{S}^{\mathcal{I}}[G], \mathbb{S}^{\mathcal{I}}[G], R\right)\right) \rightarrow B^{\bar{\lambda}}\left(\mathbb{S}, \mathbb{S}_{t}^{\mathcal{I}}[G], R\right)
$$

and is thus a weak equivalence by Lemmas 8.7 and 8.8. So $v$ is a local equivalence by two out of three. The lower horizontal map is the fibrant replacement defining $\gamma_{R}$. Finally, $u$ arises as a lift in the positive local model structure on $\mathcal{C S p}{ }_{\mathcal{R}}^{\Sigma}$

Given a map of $\mathcal{I}$-spaces $f: X \rightarrow B G$, we get a natural map

$$
\begin{aligned}
B^{\bar{\lambda}}\left(\mathbb { S } _ { \mathcal { R } } ^ { \mathcal { I } } \left[X \amalg f^{*}\right.\right. & \left.(E G), X], \mathbb{S}^{\mathcal{I}}[G], R\right) \\
& \rightarrow f^{*} B^{\bar{\lambda}}\left(\mathbb{S}_{\mathcal{R}}^{\mathcal{I}}[B G \amalg E G, B G], \mathbb{S}^{\mathcal{I}}[G], R\right) \rightarrow f^{*}\left(\gamma_{R}\right)
\end{aligned}
$$

where the first map results from the universal property of $f^{*}$ and the second map is induced by the composite $u v$ in (9.10).

LEMMA 9.12. The composite map in (9.11) is a local equivalence in $\operatorname{Sp}_{X}^{\Sigma}$.

Proof. We first suppose that $f: X \rightarrow B G$ is an absolute $\mathcal{I}$-fibration. Then the first map in (9.11) is a local equivalence by Lemmas 8.7 and 8.13 since both $\mathbb{S}^{\mathcal{I}}[G]$ 
and $R$ are flat. The second map in (9.11) is a local equivalence by Proposition 8.11 and the above observation that $u v$ is a local equivalence. Since $U(f)=f^{*}(E G)$ is a homotopy pullback, both sides send $\mathcal{I}$-equivalences to local equivalences, and the map is a local equivalence for all $f$.

Applying the collapse of base space functor $\Theta$ to (9.11) provides maps

$$
T_{E G}^{\mathcal{I}}(f) \rightarrow T_{\mathcal{R}}^{\mathcal{I}}(f) \quad \text { and } \quad T_{E G}(\tau) \rightarrow T_{\mathcal{R}}(\tau)
$$

where the second is obtained from the first by precomposing with the $\mathcal{I}$ spacification.

Proposition 9.14. The first map $T_{E G}^{\mathcal{I}} \rightarrow T_{\mathcal{R}}^{\mathcal{I}}$ in (9.13) is a natural lax symmetric monoidal stable equivalence of functors $\mathbb{S}^{\mathcal{I}} / B G \rightarrow \operatorname{Mod}_{R}$, and the second is a natural lax monoidal stable equivalence $T_{E G} \rightarrow T_{\mathcal{R}}$ of functors $\mathcal{S} /(B G)_{h \mathcal{I}} \rightarrow \operatorname{Mod}_{R}$ that respects actions of operads augmented over the BarrattEccles operad.

Proof. Since the composite $u v$ in (9.10) is a map of parametrized commutative ring spectra, $T_{E G}^{\mathcal{I}}(-) \rightarrow T_{\mathcal{R}}^{\mathcal{I}}(-)$ is a lax symmetric monoidal transformation. By the homotopy invariance of $\Theta$ and Lemma 9.12, it is a stable equivalence. The second statement then follows from the properties of the $\mathcal{I}$-spacification discussed in Section 7.6.

9.15. Thom spectra over the sphere spectrum. We now consider the case of the sphere spectrum $\mathbb{S}$, work over topological spaces, and write $F=\mathrm{GL}_{1}^{\mathcal{I}}(\mathbb{S})$ for the units of $\mathbb{S}$. (Since $\mathbb{S}$ is semistable, [6, Lemma 2.5] implies that we do not need to replace it fibrantly before forming $\mathrm{GL}_{1}^{\mathcal{I}}(\mathbb{S})$ and applying our Thom spectrum functor constructions.) In this case, $F(\mathbf{m}) \subseteq \Omega^{\mathcal{I}}(\mathbb{S})(\mathbf{m})=\Omega^{m}\left(S^{m}\right)$ is the space of self-homotopy equivalences of $S^{m}$ which is a monoid under composition. The multiplications of the $F(\mathbf{m})$ assemble to an associative and unital multiplication map $F \times F \rightarrow F$ in $\mathcal{S}^{\mathcal{I}}$. The canonical $F(\mathbf{m})$-action on the $S^{m}$ assemble to an action $F \times \mathbb{S} \rightarrow \mathbb{S}$ in $\operatorname{Sp}_{\mathcal{R}}^{\Sigma}$ where $\times$ now denotes the action introduced in (4.20). This action and the multiplication of $F$ allow us to form the two-sided bar construction

$$
B^{\times}(*, F, \mathbb{S})=\left|[q] \mapsto * \times F^{\times q} \times \mathbb{S}\right|
$$

in $\mathrm{Sp}_{\mathcal{R}}^{\Sigma}$. Its evaluation at $\mathbf{m}$ is the classifying space for sectioned fibrations with fiber equivalent to $S^{m}$ which was considered in [33, Section IX] (see also [45, Section 2]). Writing $T_{B F}^{\mathcal{I}}$ : $\operatorname{Top}^{\mathcal{I}} / B F \rightarrow \mathrm{Sp}^{\Sigma}$ for the Thom spectrum functor introduced in [45, Definition 3.3], we thus obtain a natural isomorphism 
$T_{B F}^{\mathcal{I}}(f) \cong \Theta\left(f^{*} B^{\times}(*, F, \mathbb{S})\right)$. It follows from [45, Corollaries 4.13 and 6.9] that the space level counterpart $T_{B F}=T_{B F}^{\mathcal{I}} \circ P_{B F}$ is a monoidal homotopy functor on Top $^{\mathcal{I}} / B F_{h \mathcal{I}}$ that respects actions by operads augmented over the Barratt-Eccles operad.

Our goal is to compare $T_{B F}$ to the Thom spectrum functor $T_{\mathcal{R}}$ in (9.2) with $R=\operatorname{Sing} \mathbb{S}$. We first observe that the restriction of the multiplication $F \times F \rightarrow$ $F$ of $F$ along the map $F \otimes F \rightarrow F \times F$ arising from Construction 4.21 (or from [43, Section 2.24]) provides the commutative $\mathcal{I}$-space monoid structure of $F$. A cofibrant replacement $G \rightarrow F=\mathrm{GL}_{1}^{\mathcal{I}}(\mathbb{S})$ in commutative $\mathcal{I}$-space monoids and the maps from Construction 4.21 induce comparison maps $\mathbb{S}_{t}^{\mathcal{I}}[G]^{\bar{\wedge} q} \bar{\wedge} \rightarrow$ $F^{\times q} \times \mathbb{S}$ for every $q \geqslant 0$. Using (4.23), one can check that these are compatible with the simplicial structure maps and induce a well-defined map of commutative parametrized ring spectra

$$
B^{\bar{\wedge}}\left(\mathbb{S}, \mathbb{S}_{t}^{\mathcal{I}}[G], \mathbb{S}\right) \rightarrow B^{\times}(*, F, \mathbb{S})
$$

We write $\rho: B G \rightarrow B^{\times}(F)$ for the underlying map of commutative $\mathcal{I}$-space monoids.

Proposition 9.17. The map $B^{\bar{\lambda}}\left(\mathbb{S}, \mathbb{S}_{t}^{\mathcal{I}}[G], \mathbb{S}\right) \rightarrow B^{\times}(*, F, \mathbb{S})$ is a local equivalence.

Proof. As a first step, we show that the degeneracy maps in the underlying simplicial objects are levelwise $h$-cofibrations on the base and the total spaces. For $B^{\bar{\lambda}}\left(\mathbb{S}, \mathbb{S}_{t}^{\mathcal{I}}[G], \mathbb{S}\right)$, this follows from [42, Proposition 12.7 and Lemma 7.7] and the explicit description of $\mathbb{S}_{t}^{\mathcal{I}}$ in (3.18). For $B^{\times}(*, F, \mathbb{S})$, this holds because $F$ is well based [31, Theorem 2.1].

Next we show that $\rho$ is an $\mathcal{I}$-equivalence. Since we checked that the underlying simplicial object of base $\mathcal{I}$-spaces is good, it is sufficient to show that for fixed $q$, the map of $\mathcal{I}$-spaces $G^{\boxplus q} \rightarrow F^{\times q}$ is an $\mathcal{I}$-equivalence. For $q=2$, it can be identified as the composite of the morphism $\rho_{G, G}: G \otimes G \rightarrow G \times G$ considered in [43, Section 2.24] and the level equivalence $G \times G \rightarrow F \times F$ induced by $\rho$. Since $G$ is semistable and flat by construction, $\rho_{G, G}$ is an $\mathcal{I}$-equivalence by [43, Proposition 2.27]. The assertion for $q>2$ follows by an inductive argument based on [43, Proposition 2.27].

To check that (9.16) is a local equivalence, we form a commutative diagram

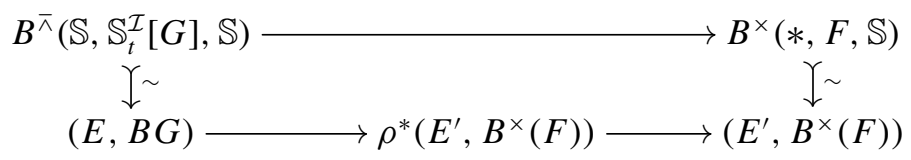


where the vertical maps are fibrant replacements in the absolute local model structures on $\mathrm{Sp}_{B G}^{\Sigma}$ and $\mathrm{Sp}_{B^{\times}(F)}^{\Sigma}$ and the lower left-hand horizontal map arises by extending the resulting map $B^{\bar{\wedge}}\left(\mathbb{S}, \mathbb{S}_{t}^{\mathcal{I}}[G], \mathbb{S}\right) \rightarrow \rho^{*}\left(E^{\prime}, B^{\times}(F)\right)$ in $\operatorname{Sp}_{B G}^{\Sigma}$ over the left-hand acyclic cofibration. By Corollary 6.9, it is sufficient to show that the map of fibrant objects $(E, B G) \rightarrow \rho^{*}\left(E^{\prime}, B^{\times}(F)\right)$ is a local equivalence in $\operatorname{Sp}_{B G}^{\Sigma}$.

Let $\iota: * \rightarrow B G$ be the unit. As in the discussion preceding Proposition 9.8, we may assume that $G$ is the realization of a cofibrant replacement of $\mathrm{GL}_{1}^{\mathcal{I}}(\operatorname{Sing} \mathbb{S})$ and that $\mathbb{S}^{\mathcal{I}}[G] \rightarrow \mathbb{S}$ factors through $|\operatorname{Sing} \mathbb{S}| \rightarrow \mathbb{S}$. Togetherwith the above statement about $h$-cofibrations, this implies that the map

$$
B^{\bar{\lambda}}\left(\mathbb{S}, \mathbb{S}_{t}^{\mathcal{I}}[G],|\operatorname{Sing} \mathbb{S}|\right) \rightarrow B^{\bar{\wedge}}\left(\mathbb{S}, \mathbb{S}_{t}^{\mathcal{I}}[G], \mathbb{S}\right)
$$

is an absolute level equivalence. Using this, it follows from Proposition 8.5 that the canonical map $\mathbb{S} \rightarrow \iota^{*}(E, B G)$ is a stable equivalence. To get an analogous statement for $\rho^{*}\left(E^{\prime}, B^{\times}(F)\right)$, we use the absolute $\mathcal{I}$-model structure and the standard levelwise replacement by a Hurewicz fibration to factor $\rho \iota$ as an $\mathcal{I}$ equivalence $j: * \rightarrow X$ followed by a map $q: X \rightarrow B^{\times}(F)$ that is both an absolute $\mathcal{I}$-fibration and a levelwise Hurewicz fibration. This factorization gives rise to a commutative square

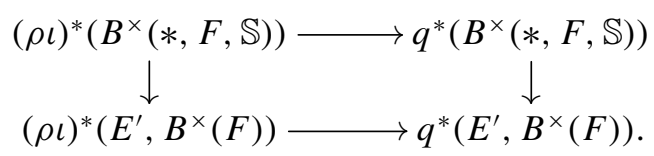

The right-hand vertical map is a local equivalence by Proposition 8.11 and thus a stable equivalence after applying $\mathbb{L} \Theta$. The top horizontal map is a stable equivalence after applying $\mathbb{L} \Theta$ by [45, Theorem 1.4] (where the $T$-goodness assumption is taken care of by [45, Lemmas 2.2 and 2.3]). Since $j$ is an $\mathcal{I}$ equivalence and $\left(E^{\prime}, B^{\times}(F)\right)$ is fibrant, Corollary 6.9 implies that the bottom horizontal map is a local equivalence. Hence, $\mathbb{S} \cong(\rho \iota)^{*}\left(B^{\times}(*, F, \mathbb{S})\right) \rightarrow$ $(\rho \iota)^{*}\left(E^{\prime}, B^{\times}(F)\right)$ is a stable equivalence.

It follows that $(E, B G) \rightarrow \rho^{*}\left(E^{\prime}, B^{\times}(F)\right)$ induces a stable equivalence after pullback along $\iota$. Since the fibers are $\Omega$-spectra, the induced map of fibers is even a level equivalence. By inspecting the simplicial object defining $B G$, each $B G(\mathbf{n})$ is connected. The long exact sequences of homotopy groups of the Serre fibrations $E_{n} \rightarrow B G(\mathbf{n})$ and $\rho(\mathbf{n})^{*} E_{n}^{\prime} \rightarrow B G(\mathbf{n})$ show that $(E, G) \rightarrow \rho^{*}\left(E^{\prime}, B^{\times}(F)\right)$ is a level equivalence and hence a local equivalence.

We now explain how the proposition leads to a comparison of Thom spectrum functors. Let $R=\operatorname{Sing} \mathbb{S}$ and let $G \rightarrow \mathrm{GL}_{1}^{\mathcal{I}} R$ be a cofibrant replacement of its $\mathcal{I}$-space units. (This is homotopically meaningful since $R$ is level fibrant and 
semistable.) By the discussion before Proposition 9.8, we get a map $\mathbb{S}^{\mathcal{I}}[|G|] \rightarrow \mathbb{S}$ and note that $|G|$ can be used as a model for the cofibrant replacement of the units of the sphere spectrum in topological spaces.

Next we consider $\bar{\gamma}_{\mathbb{S}}$, the bar resolution of $B^{\bar{\lambda}}\left(\mathbb{S}, \mathbb{S}_{t}^{\mathcal{I}}[G], R\right)$. It is the object in $\mathrm{Sp}_{\mathcal{R}}^{\Sigma}$ defined by

$$
\left(\bar{\gamma}_{\mathbb{S}}\right)_{n}=\operatorname{hocolim}_{\alpha: \mathbf{k} \rightarrow \mathbf{n}} B^{\bar{\wedge}}\left(\mathbb{S}, \mathbb{S}_{t}^{\mathcal{I}}[G], R\right)_{k} \bar{\wedge} S^{\mathbf{n}-\alpha}
$$

where the homotopy colimit is taken over the category $\mathcal{I} \downarrow \mathbf{n}$. The base $\mathcal{I}$-space of $\bar{\gamma}_{\mathbb{S}}$ is the bar resolution of $B G$ used in the $\mathcal{I}$-spacification. As in the case of symmetric spectra (see [7, Section 7.3]), there is a canonical level equivalence $\bar{\gamma}_{\mathbb{S}} \rightarrow B^{\bar{\wedge}}\left(\mathbb{S}, \mathbb{S}_{t}^{\mathcal{I}}[G], R\right)$. Applying realization, composing this map with the fibrant replacement defining $\gamma_{R}$, and using Proposition 9.17 give a zig-zag of local equivalences

$$
\left|\gamma_{R}\right| \leftarrow\left|\bar{\gamma}_{\mathbb{S}}\right| \rightarrow B^{\times}(*, F, \mathbb{S})
$$

Let $\tau: K \rightarrow B G_{h \mathcal{I}}$ be a map of simplicial sets. We note that its $\mathcal{I}$-spacification $\tau_{\mathcal{I}}: P_{\tau}(K) \rightarrow B G$ factors by definition as an absolute $\mathcal{I}$-fibration $\bar{\tau}_{\mathcal{I}}: P_{\tau}(K) \rightarrow$ $\overline{B G}$ and the canonical map $t: \overline{B G} \rightarrow B G$. This factorization, the maps (9.18), and the map from Lemma 7.8 give rise to a zig-zag

$$
\left|T_{\mathcal{R}}(\tau)\right|=\left|T_{\mathcal{R}}^{\mathcal{I}}\left(\tau_{\mathcal{I}}\right)\right| \leftarrow\left|\Theta\left(\bar{\tau}_{I}^{*}\left(\bar{\gamma}_{\mathbb{S}}\right)\right)\right| \rightarrow T_{B F}^{\mathcal{I}}\left(\left(\rho_{h \mathcal{I}} \circ|\tau|\right)_{\mathcal{I}}\right)=T_{B F}\left(\rho_{h \mathcal{I}} \circ|\tau|\right) .
$$

Here we use the bar resolution in the middle term since $\bar{\tau}_{\mathcal{I}}$ being a fibration ensures that $\bar{\tau}_{I}^{*}\left(\bar{\gamma}_{\mathbb{S}}\right)$ captures a well-defined homotopy type without $\bar{\gamma}_{\mathbb{S}}$ being locally fibrant.

Proposition 9.20. The maps in (9.19) are natural monoidal stable equivalences that respect actions of operads augmented over the Barratt-Eccles operad.

Proof. The claim about monoidality and operad actions is clear since all constructions involved preserve these structures. To see that the first map in (9.19) is a stable equivalence, we factor $\tau_{\mathcal{I}}$ as an $\mathcal{I}$-equivalence followed by an absolute fibration and use Corollary 6.9, Proposition 8.11, and the fact that the $\Theta$ in simplicial sets sends local equivalences to stable equivalences. For the second map, we argue as in the proof of the previous proposition, factor $\left(\rho_{h \mathcal{I}} \circ|\tau|\right)_{\mathcal{I}}$ as an $\mathcal{I}$-equivalence followed by a map that is both an absolute $\mathcal{I}$-fibration and a levelwise Hurewicz fibration, and apply Proposition 9.17, the $\mathcal{I}$-equivalence part of Lemma 7.8, Proposition 8.11, and [45, Theorem 1.4]. 
Together with the $\infty$-categorical comparison to be proved in Section 10.12, these results now combine to give the statement of Theorem 1.8.

Proof of Theorem 1.8. This is a combination of Propositions 9.8, 9.14, and 9.20, the $\infty$-categorical comparison in Lemma 10.3, and Proposition 10.15.

\section{Comparison to $\infty$-categorical parametrized spectra}

If $\mathcal{C}$ is a model category, we write $\mathcal{C}_{\infty}$ for the underlying $\infty$-category of $\mathcal{C}$. In particular, we write $\mathcal{S}_{\infty}$ (respectively $\mathrm{Sp}_{\infty}$ ) for the $\infty$-category of spaces (respectively spectra). When $\mathcal{C}$ is a symmetric monoidal model category, then $\mathcal{C}_{\infty}$ inherits the structure of a symmetric monoidal $\infty$-category from $\mathcal{C}$ [35, Example 4.1.7.6]. More specifically, the localization functor $\mathcal{C} \rightarrow \mathcal{C}_{\infty}$ is lax symmetric monoidal and strong symmetric monoidal when restricted to cofibrant objects; see [22, Proposition 3.2.2] and also [40, Appendix A].

10.1. Comparison of categories. The next lemma is closely related to [1, Proposition B.1 and Theorem B.4].

LEMMA 10.2. Let $X$ be an $\mathcal{I}$-space. Then the $\infty$-category $\left(\operatorname{Sp}_{X}^{\Sigma}\right)_{\infty}$ resulting from the local model structure on $\operatorname{Sp}_{X}^{\Sigma}$ is equivalent to $\operatorname{Fun}\left(X_{h \mathcal{I}}, \mathrm{Sp}_{\infty}\right)$, the $\infty$-category of functors from the underlying $\infty$-groupoid of $X_{h \mathcal{I}}$ to the $\infty$-category of spectra.

Proof. By [35, Remark 1.4.2.9], stabilization commutes with the passage to presheaf categories. Hence, $\operatorname{Fun}\left(X_{h \mathcal{I}}, \operatorname{Sp}_{\infty}\right)$ is equivalent to $\operatorname{Sp}\left(\operatorname{Fun}\left(X_{h \mathcal{I}}, \mathcal{S}_{\infty}\right)\right)$, the stabilization of the category of space-valued functors on $X_{h \mathcal{I}}$. The $\infty$-category $\operatorname{Fun}\left(X_{h \mathcal{I}}, \mathcal{S}_{\infty}\right)$ is equivalent to $\mathcal{S}_{\infty} / X_{h \mathcal{I}} \simeq\left(\mathcal{S} / X_{h \mathcal{I}}\right)_{\infty}$ by [34, Theorem 2.2.1.2]. The fact that stabilization commutes with the passage to underlying $\infty$-categories and [26, Corollary 10.4] imply that $\operatorname{Sp}\left(\left(\mathcal{S} / X_{h \mathcal{I}}\right)_{\infty}\right)$ is equivalent to $\left(\operatorname{Sp}_{X_{h \mathcal{I}}}^{\Sigma}\right)_{\infty}$. The claim follows from the Quillen equivalence between $\mathrm{Sp}_{X}^{\Sigma}$ and $\mathrm{Sp}_{X_{h \mathcal{I}}}^{\Sigma}$ established in Corollary 5.24.

In the next step, we show that the equivalence in the last lemma is natural so that we can identify the functors between $\operatorname{Sp}_{X}^{\Sigma}$ and $\operatorname{Sp}_{Y}^{\Sigma}$ induced by $f: X \rightarrow Y$ with their $\infty$-categorical counterparts including coherences between compositions.

LEMMA 10.3. The functors $\left(\mathcal{S}^{\mathcal{I}}\right)_{\infty} \rightarrow \mathrm{Cat}_{\infty}$ given by $\operatorname{Fun}\left((-)_{h \mathcal{I}}, \mathrm{Sp}_{\infty}\right)$ and $\left(\mathrm{Sp}_{(-)}^{\Sigma}\right)_{\infty}$ are equivalent. 
Proof. Our strategy is to show that they classify equivalent bicartesian fibrations. For this, we first show that (up to replacement by categorical fibrations) the functors

$$
\left(\mathrm{Sp}_{\mathcal{R}}^{\Sigma}\right)_{\infty} \stackrel{u}{\rightarrow} \operatorname{Ar}\left(\mathcal{S}^{\mathcal{I}}\right)_{\infty} \stackrel{p}{\rightarrow}\left(\mathcal{S}^{\mathcal{I}}\right)_{\infty}
$$

induced by the right Quillen functors $\Omega_{\mathrm{ar}}^{\mathcal{I}}=\pi_{\mathrm{ar}}^{\mathcal{I}} \circ \Omega_{\mathcal{R}}^{\mathcal{I}}$ and $\pi_{b}$ (see Corollary 5.13 and Lemmas 5.12 and 5.14) exhibit $u$ as the stable envelope of $p$ in the sense of [35, Definition 7.3.1.1]. For [35, Definition 7.3.1.1(1)], we note that both $p$ and $p u$ are presentable fibrations because the fibers are the underlying $\infty$ categories of combinatorial model categories (using the simplicial version of our categories) and they are cartesian and cocartesian by [20, Proposition 3.1.2] and (the dual of) [34, Corollary 5.2.2.5]. For the condition [35, Definition 7.3.1.1(2)], we need the following observation: By [34, Proposition 2.4.4.3], a morphism $(f, g):(E, X) \rightarrow\left(E^{\prime}, X^{\prime}\right)$ in $\left(\operatorname{Sp}_{\mathcal{R}}^{\Sigma}\right)_{\infty}$ is $p u$-cartesian if (after (co)fibrant replacement of source and target, respectively) the induced map $(f$, id $):(E, X)$ $\rightarrow g^{*}\left(E^{\prime}, X^{\prime}\right)$ is a local equivalence and similarly for the case of $p$-cartesian morphisms. But this condition is preserved by $u$ since $g^{*} \pi_{\mathrm{ar}}^{\mathcal{I}} \Omega_{X^{\prime}}^{\mathcal{I}} \cong \Omega_{X}^{\mathcal{I}} \pi_{\mathrm{ar}}^{\mathcal{I}} g^{*}$ for any map $g: X \rightarrow X^{\prime}$ and all functors involved are right Quillen. For [35, Definition 7.3.1.1(3)], we note that [35, Example 7.3.1.4] and Lemma 5.21 imply that $u$ restricts to a stable envelope on fibers.

Now,

$$
\int_{\mathcal{S}_{\infty}} \operatorname{Fun}\left(-, \operatorname{Sp}_{\infty}\right) \stackrel{\int \Omega^{\infty}}{\longrightarrow} \int_{\mathcal{S}_{\infty}} \operatorname{Fun}\left(-,\left(\mathcal{S}_{\infty}\right)_{*}\right) \stackrel{\text { pr }}{\rightarrow} \mathcal{S}_{\infty}
$$

is also well known to be a stable envelope. Thus, the uniqueness theorem [35, Proposition 7.3.1.7(3)] reduces the claim to the corresponding space level statement. The latter is obtained from the Quillen equivalence

$$
\operatorname{colim}_{\mathcal{I}}: \mathcal{S} \rightleftarrows \mathcal{S}^{\mathcal{I}}: \text { const }_{\mathcal{I}}
$$

and the equivalences

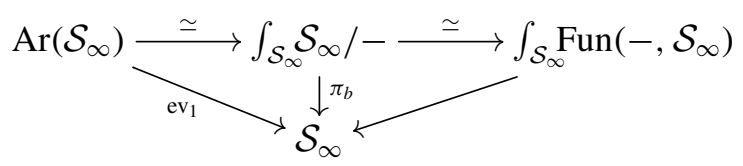

of bicartesian fibrations where the right equivalence follows from the unstraightening equivalence [34, Theorem 2.2.1.2], and the left is immediate from the main theorem of [20] together with the compatibility between diagram categories of model and $\infty$-categories. 
Next we give an $\infty$-categorical interpretation of the category of symmetric spectra in retractive spaces $\mathrm{Sp}_{\mathcal{R}}^{\Sigma}$. To do so, let us denote

$$
T \mathcal{S}_{\infty}=\int_{\mathcal{S}_{\infty}} \operatorname{Fun}\left(-, \mathrm{Sp}_{\infty}\right)
$$

the $\infty$-categorical Grothendieck construction of $\operatorname{Fun}\left(-, \mathrm{Sp}_{\infty}\right): \mathcal{S}_{\infty} \rightarrow \mathrm{Cat}_{\infty}$, which is a model for the tangent bundle of the category $\mathcal{S}_{\infty}$ as in $[35$, Section 7.3.1].

PROPOSITION 10.4. The $\infty$-category $\left(\mathrm{Sp}_{\mathcal{R}}^{\Sigma}\right)_{\infty}$ resulting from the local model structure on $\mathrm{Sp}_{\mathcal{R}}^{\Sigma}$ is canonically equivalent to $T \mathcal{S}_{\infty}$.

Proof. Combining [20, Proposition 3.1.2], Theorem 6.7, and Lemma 10.2, we see that the $\infty$-category $\left(\mathrm{Sp}_{\mathcal{R}}^{\Sigma}\right)_{\infty}$ is equivalent to the $\infty$-categorical Grothendieck construction of $\left(\operatorname{Sp}_{(-)}^{\Sigma}\right)_{\infty}:\left(\mathcal{S}^{\mathcal{I}}\right)_{\infty} \rightarrow \mathrm{Cat}_{\infty}$. By Lemma 10.3 , we can identify it with the $\infty$-categorical Grothendieck construction of

$$
\operatorname{Fun}\left(-, \mathrm{Sp}_{\infty}\right):\left(\mathcal{S}^{\mathcal{I}}\right)_{\infty} \rightarrow \mathrm{Cat}_{\infty}
$$

The claim follows because $\mathcal{S}^{\mathcal{I}}$ and $\mathcal{S}$ are Quillen equivalent [42, Theorem 3.3].

10.5. Comparison of symmetric monoidal categories. When $\mathcal{C}$ is a symmetric monoidal $\infty$-category, then the Day convolution product gives rise to a symmetric monoidal structure on $\operatorname{Fun}\left(\mathcal{C}, \mathcal{S}_{\infty}\right)$; see [16]. Using [15, Theorem 5.1] and the equivalence $\operatorname{Sp}\left(\operatorname{Fun}\left(\mathcal{C}, \mathcal{S}_{\infty}\right)\right) \simeq \operatorname{Fun}\left(\mathcal{C}, \operatorname{Sp}_{\infty}\right)$, we thus get a uniquely determined symmetric monoidal structure on $\operatorname{Fun}\left(\mathcal{C}, \mathrm{Sp}_{\infty}\right)$.

If $M$ is a commutative $\mathcal{I}$-space monoid, then $M_{h \mathcal{I}}$ inherits an action of the Barratt-Eccles operad [45, Proposition 6.5]. By [39, Proposition 4.1], the underlying $\infty$-groupoid of $M_{h \mathcal{I}}$ represents a symmetric monoidal $\infty$ groupoid, and $\operatorname{Fun}\left(M_{h \mathcal{I}}, \mathrm{Sp}_{\infty}\right)$ is a symmetric monoidal $\infty$-category by the above discussion.

THEOREM 10.6. Let $M$ be a commutative $\mathcal{I}$-space monoid. Then the symmetric monoidal $\infty$-category $\left(\mathrm{Sp}_{M}^{\Sigma}\right)_{\infty}$ resulting from the absolute or positive local model structures on $\mathrm{Sp}_{M}^{\Sigma}$ and $\operatorname{Fun}\left(M_{h \mathcal{I}}, \mathrm{Sp}_{\infty}\right)$ are equivalent as symmetric monoidal $\infty$ categories.

REMARK 10.7. There is a functorial rigidification $(-)^{\text {rig }}$ of $E_{\infty}$ spaces (in their incarnation as spaces with an action of the Barratt-Eccles operad) to commutative 
$\mathcal{I}$-space monoids so that $M \simeq\left(M^{\mathrm{rig}}\right)_{h \mathcal{I}}$ as $E_{\infty}$ spaces [42, Corollary 3.7]. In this situation, the previous theorem implies that $\mathrm{Sp}_{M^{\text {rig }}}^{\Sigma}$ represents the symmetric monoidal $\infty$-category $\operatorname{Fun}\left(M, \mathrm{Sp}_{\infty}\right)$.

The existence of a rigidification of $\operatorname{Fun}\left(M, \mathrm{Sp}_{\infty}\right)$ is also implied by [39, Theorem 1.1], but the above construction provides a smaller model.

Proof of Theorem 10.6. We show that the assignments $M \mapsto \operatorname{Fun}\left(M_{h \mathcal{I}}, \operatorname{Sp}_{\infty}\right)$ and $M \mapsto\left(\operatorname{Sp}_{M}^{\Sigma}\right)_{\infty}$ are equivalent as functors $\left(\mathcal{C S}^{\mathcal{I}}\right)_{\infty} \rightarrow$ SymMonCat $_{\infty}$. By Lemma 10.3 , they are equivalent as functors to $\mathrm{Cat}_{\infty}$. So it remains to compare the symmetric monoidal structures. For this, we adapt the argument given in the proof of [39, Proposition 2.4]. The only change that is necessary to apply it in the case at hand is that we have to argue with the forgetful functor $U:\left(\mathcal{C S}^{\mathcal{I}}\right)_{\infty} \rightarrow \mathcal{S}_{\infty}$ rather than with the one SymMonCat ${ }_{\infty} \rightarrow \mathrm{Cat}_{\infty}$.

In order to compare our construction of the universal bundle $\gamma_{R}$ with that of [2], we also have to compare the monoidal structures on $\operatorname{Sp}_{\mathcal{R}}^{\Sigma}$ and $T \mathcal{S}_{\infty}$. We are, however, not aware of a construction of the requisite symmetric monoidal structures on tangent categories in the literature ([35, Example 7.3.1.15] only gives the cartesian monoidal structure on $T \mathcal{S}_{\infty}$ ). To describe it, consider therefore the following general situation: Suppose we are given cocartesian fibrations of $\infty$-operads

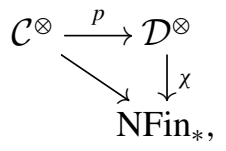

admitting finite operadic limits. In particular, by definition, $\mathcal{C}$ and $\mathcal{D}$ are then symmetric monoidal $\infty$-categories (see [35, Example 2.1.2.18]). The example of relevance for us is $\mathcal{D}^{\otimes}=\mathcal{S}_{\infty}^{\times}$and $\mathcal{C}^{\otimes}=\operatorname{Ar}\left(\mathcal{S}_{\infty}\right)^{\times}$with $p$ the projection to the base space. We are then looking for a left exact map of $\infty$-operads $u: \mathcal{E}^{\otimes} \rightarrow \mathcal{C}^{\otimes}$, that is, a lax symmetric monoidal functor, that exhibits $\mathcal{E}^{\otimes}$ as a fiberwise stabilization of $p$ in the following sense. We require that $\mathcal{E}$ is a stable $\mathcal{D}$-monoidal $\infty$-category in the sense of [35, Definition 7.3.4.1], and for all other stable $\mathcal{D}$-monoidal $\infty$ categories $\mathcal{B}$, postcomposition with $u$ induces an equivalence

$$
\mathrm{Op}_{/ \mathcal{D}}^{\text {lex }}(\mathcal{B}, \mathcal{E}) \longrightarrow \mathrm{Op}_{/ \mathcal{D}}^{\text {lex }}(\mathcal{B}, \mathcal{C})
$$

where we have used $O p^{\text {lex }}$ to denote the category of operads with finite operadic limits and operad maps preserving these (as in [38, Definition 2.3]). The case $\mathcal{D}=$ $p t$ (that is, $\mathcal{D}^{\otimes}=\mathrm{NFin}_{*}, \chi=\mathrm{id}$ ) is extensively discussed in both [35, Sections 6.2.4-6.2.6] and [38, Section 4]. The construction in [35, Section 6.2.5] provides a candidate $u: \mathrm{St}_{\chi}(p) \rightarrow \mathcal{C}^{\otimes}$ for such a fiberwise stabilization also in our case. We will review this construction in the next proof. 
Already for $\chi=\mathrm{id}$, however, the construction in general does not provide a map $u$ such that $p u$ is cocartesian but rather only locally so, for example, if $\mathcal{C}$ is the category of pointed spaces under the cartesian product; see [35, Example 6.2.4.17].

A useful criterion for $p u$ to be cocartesian is established in [38, Proposition 4.9]. This result readily generalizes to give the following.

Proposition 10.8. Let $p: \mathcal{C}^{\otimes} \rightarrow \mathcal{D}^{\otimes}$ be a cocartesian fibration of $\infty$ operads between symmetric monoidal $\infty$-categories. Assume further that $\mathcal{C}$ is differentiable and that for any multimorphism $\left(d_{1}, \ldots, d_{n}\right) \rightarrow d^{\prime}$ in $\mathcal{D}^{\otimes}$, the induced functor $\mathcal{C}_{d_{1}} \times \cdots \times \mathcal{C}_{d_{n}} \rightarrow \mathcal{C}_{d^{\prime}}$ commutes with colimits in each variable.

Then pu: $\mathrm{St}_{\chi}(p) \rightarrow \mathcal{D}^{\otimes}$ makes $\mathrm{St}_{\chi}(p)$ a cocartesian fibration of $\infty$-operads. In particular, the composite $\chi$ pu makes $\mathrm{St}_{\chi}(p)$ into a symmetric monoidal $\infty$ category and $u: \mathrm{St}_{\chi}(p) \rightarrow \mathcal{C}^{\otimes}$ is a map of $\infty$-operads, that is, it is lax symmetric monoidal. Furthermore, the $\mathcal{D}$-algebra structure on $\mathrm{St}_{\chi}(p)$ satisfies the same commutation with colimits as that of $\mathcal{C}$; in particular, the symmetric monoidal structure commutes with colimits in each variable. Finally, $u$ exhibits $\mathrm{St}_{\chi}(p)$ as a fiberwise stabilization of $p$ in the sense above.

Note that as cocartesian fibrations, both $p$ and $p u$ automatically preserve cocartesian lift of morphisms in $\mathrm{NFin}_{*}$ and are therefore strong symmetric monoidal.

REMARK 10.9. Contrary to another claim in [35, Example 6.2.4.17], [38, Proposition 4.9] or [35, Examples 6.2.1.5 or 6.2.3.28] implies that the composite $p u$ is a cocartesian fibration for $\mathcal{C}^{\otimes}=\left(\mathcal{S}_{*}\right)_{\infty}^{\wedge}$, the category of pointed spaces with the smash product.

Proof of Proposition 10.8. Let us recall the definition of $\mathrm{St}_{\chi}(p)$ from [35, Construction 6.2.5.20]. Consider first the simplicial set $q: P \mathrm{St}_{\chi}(p) \rightarrow \mathcal{D}^{\otimes}$ given by the universal property that for all simplicial sets $K$ over $\mathcal{D}^{\otimes}$, we have

$$
\operatorname{Hom}_{\mathcal{D}^{\otimes}}\left(K, P \mathrm{St}_{\chi}(p)\right) \cong \operatorname{Hom}_{\mathcal{D}^{\otimes}}\left(K \times_{\mathrm{NFin}_{*}}\left(\mathcal{S}_{*}^{\mathrm{fin}}\right)^{\wedge}, \mathcal{C}^{\otimes}\right) .
$$

Then $D \mathrm{St}_{\chi}(p)$ is given as the full simplicial subset of $P \mathrm{St}_{\chi}(p)$ spanned by those vertices $v$ that correspond to product functors

$$
\prod_{\chi q(v) \backslash\{*\}} \mathcal{S}_{*}^{\mathrm{fin}} \simeq\left(\mathcal{S}_{*}^{\mathrm{fin}}\right)_{\chi q(v)}^{\wedge} \longrightarrow \mathcal{C}_{q(v)}^{\otimes} \simeq \prod_{i \in \chi q(v) \backslash\{*\}} \mathcal{C}_{q_{i}(v)}
$$

and $\mathrm{St}_{\chi}(p)$ is spanned by those vertices corresponding to product functors whose factors are reduced and excisive. The structure map $u: D \mathrm{St}_{\chi}(p) \subseteq P \mathrm{St}_{\chi}(p) \rightarrow$ 
$\mathcal{C}^{\otimes}$ is given by taking $K=P \operatorname{St}_{\chi}(p) \rightarrow \mathcal{D}^{\otimes}$ in the universal property above and precomposing the map corresponding to the identity with the section $\mathrm{NFin}_{*} \rightarrow$ $\left(\mathcal{S}_{*}^{\mathrm{fin}}\right)^{\wedge}$ that witnesses the $E_{\infty}$-structure of the unit $S^{0}$. By construction, we have $q=p u$.

Now the restriction of $q$ to $D \operatorname{St}_{x}(p)$ is a cocartesian fibration just as in [16, Lemma 2.10] (which treats the case $\mathcal{D}=p t$ ). It follows that in order to recognize $D \mathrm{St}_{\chi}(p)$ as a symmetric monoidal category, we only have to verify the Segal condition for $\chi q$, but this is immediate (compare [16, Proposition 2.11]). By definition, $q$ is then a cocartesian fibration of operads. Part (2) of [35, Proposition 2.2.1.9] (which should have $\mathcal{O}^{\otimes}$ instead of $\mathrm{NFin}_{*}$ as the target of $p \mid \mathcal{D}^{\otimes}$ ) applied to the localization functor provided by [35, Theorem 6.1.1.10] (precomposed with reduction) therefore implies the same for the restriction of $q$ to $\mathrm{St}_{\chi}(p)$, as desired; the assumptions of [35, Theorem 2.1.1.9] follow from the chain rule for the first derivative [35, Theorem 6.2.1.22]. That $u$ is lax symmetric monoidal follows just as in [38, Corollary 3.8], and for the restriction to $\operatorname{St}_{\chi}(p)$, it then follows from [35, Proposition 2.2.1.9(3)]. Commutation with colimits for the operad structure on $D \mathrm{St}_{\chi}(p)$ can be verified as in [16, Lemma 2.13], and for $\mathrm{St}_{\chi}(p)$, it follows from [35, Proposition 2.2.1.9(3)].

Finally, to see that $u$ indeed exhibits $\operatorname{St}_{\chi}(p)$ as a stabilization of $p$, we note that $\mathrm{St}_{\chi}(p)$ is stable by part (3) of [35, Theorem 6.2.5.25], and, in particular, $u$ is left exact. To finish the proof, we invoke part (3) of [35, Theorem 6.2.6.6] and need to check that a left exact decomposition-stable functor $F: \mathcal{E}^{\otimes} \rightarrow \mathrm{St}_{\chi}(p)$ with fiberwise stable source is a map of $\infty$-operads if and only $u F: \mathcal{E}^{\otimes} \rightarrow \mathcal{C} \otimes$ is. But this can be shown almost verbatim as in the proof of [35, Theorem 6.2.6.2] on the same page.

We apply the above to the cocartesian fibration of operads $\pi_{c}: \operatorname{Ar}\left(\mathcal{S}_{\infty}\right)^{\times} \longrightarrow$ $\mathcal{S}_{\infty}^{\times}$projecting to the codomain. It produces a symmetric monoidal structure on $T \mathcal{S}_{\infty}$ that models the exterior smash product $\bar{\wedge}$.

THEOREM 10.10. The categories $\left(\mathrm{Sp}_{\mathcal{R}}^{\Sigma}\right)_{\infty}$ and $T \mathcal{S}_{\infty}$ are canonically equivalent as stable $\left(\mathcal{S}_{\infty}\right)^{\times}$-monoidal categories and thus, in particular, as symmetric monoidal $\infty$-categories.

Proof. To avoid confusion, we shall denote the monoidal structure on $T \mathcal{S}_{\infty}$ by the generic $\otimes$ with unit $\mathbf{1}$ in the present proof.

Let $\left(\mathcal{S}^{\mathcal{I}}\right)_{\infty}^{\bigotimes}$ denote the $\infty$-operad underlying the symmetric monoidal structure on $\mathcal{S}^{\mathcal{I}}$ given by the $\bigotimes$-product. By Propositions 5.19 and 5.11, $\pi_{b}:\left(\operatorname{Sp}_{\mathcal{R}}^{\Sigma}\right)_{\infty} \rightarrow$ $\left(\mathcal{S}^{\mathcal{I}}\right)_{\infty}^{\bigotimes}$ exhibits $\left(\operatorname{Sp}_{\mathcal{R}}^{\Sigma}\right)_{\infty}^{\bar{\lambda}}$ as a stable $\left(\mathcal{S}^{\mathcal{I}}\right)_{\infty}^{\bigotimes}$-monoidal category. By Lemmas 4.9, 5.12, and 5.14, the functor $\left(\Omega_{\mathrm{ar}}^{\mathcal{I}}\right)_{\infty}:\left(\operatorname{Sp}_{\mathcal{R}}^{\Sigma}\right)_{\infty}^{\wedge} \rightarrow \operatorname{Ar}\left(\mathcal{S}^{\mathcal{I}}\right)_{\infty}^{\otimes}$ is left exact and a map 
of $\infty$-operads, that is, lax symmetric monoidal. From the equivalence $\left(\mathcal{S}^{\mathcal{I}}\right)_{\infty}^{\bigotimes} \simeq$ $\mathcal{S}_{\infty}^{\times}$and Proposition 10.8, we therefore obtain a left exact map of $\infty$-operads

$$
c:\left(\mathrm{Sp}_{\mathcal{R}}^{\Sigma}\right)_{\infty}^{\bar{\lambda}} \longrightarrow T \mathcal{S}_{\infty}^{\otimes}
$$

that is, a lax symmetric monoidal functor. By the universal property of stabilizations, it agrees with that from Proposition 10.4 when restricted to $\left(\mathrm{Sp}_{\mathcal{R}}^{\Sigma}\right)_{\infty}$. Once we show that $c$ is in fact strong symmetric monoidal, then [35, Remark 2.1.3.8] implies that it is an equivalence of symmetric monoidal $\infty$ categories.

To see the latter, we need to verify that the canonical maps

$$
\mathbf{1} \rightarrow c(\mathbb{S}) \quad \text { and } \quad c(X) \otimes c(Y) \rightarrow c(X \bar{\wedge} Y)
$$

are equivalences, where 1 denotes the unit of $T \mathcal{S}_{\infty}$. To do so, we note that the functor $\Omega^{\infty}: T \mathcal{S}_{\infty} \rightarrow \operatorname{Ar}\left(\mathcal{S}_{\infty}\right)$ admits a strong symmetric monoidal adjoint $\Sigma_{+}^{\infty}$. To construct it, recall the map of $\infty$-operads $u: D \mathrm{St}_{\chi}(t) \rightarrow \operatorname{Ar}\left(\mathcal{S}_{\infty}\right)^{\times}$from the previous proof. We then invoke [35, Corollary 7.3.2.12] for $u$ and [35, Proposition 2.2.1.9] for the restriction to the localization $T \mathcal{S}_{\infty}=\mathrm{St}_{\chi}\left(\mathrm{ev}_{1}\right)$ of $D \mathrm{St}_{\chi}\left(\mathrm{ev}_{1}\right)$. Their assumptions are verified just as in [38, Corollary 3.8 and Proposition 4.9]. This argument immediately shows that the map between unit objects is an equivalence as the sphere $\mathbb{S} \in \operatorname{Sp}_{\mathcal{R}}^{\Sigma}$ is also given by the left adjoint $\mathbb{S}_{\mathcal{R}}^{\mathcal{I}} \circ l_{\text {ar }}^{\mathcal{I}}: \operatorname{Ar}\left(\mathcal{S}^{\mathcal{I}}\right) \rightarrow \operatorname{Sp}_{\mathcal{R}}^{\Sigma}$ evaluated on the unit $* \rightarrow *$ of $\operatorname{Ar}\left(\mathcal{S}^{\mathcal{I}}\right)$. The second claim follows since the class of pairs of spectra, for which the map in question is an equivalence, is closed under colimits in either variable and contains pairs of suspension spectra by the monoidality of $\Sigma_{+}^{\infty}$.

REMARK 10.11. When applied to the example of the target fibration

$$
\operatorname{Ar}\left(\operatorname{Alg}_{E_{\infty}}\left(\operatorname{Sp}_{\infty}\right)\right)^{\otimes} \longrightarrow \operatorname{Alg}_{E_{\infty}}\left(\operatorname{Sp}_{\infty}\right)^{\otimes}
$$

considered in detail in [35, Section 7.3], the symmetric monoidal structure from Proposition 10.8 on $T\left(\operatorname{Alg}_{E_{\infty}}\left(\operatorname{Sp}_{\infty}\right)\right)$ is also readily identified: under the equivalence of $T\left(\operatorname{Alg}_{E_{\infty}}\left(\operatorname{Sp}_{\infty}\right)\right)$ with the category of all modules over $E_{\infty}$-ring spectra [35, Theorem 7.3.4.18], it corresponds to the smash product on both the rings and the modules.

We have now verified all parts of the $\infty$-categorical theorem from Section 1 .

Proof of Theorem 1.6. This is a combination of Lemma 10.3, Proposition 10.4, and Theorems 10.6 and 10.10. 
10.12. Comparison of universal bundles. In order to formulate the comparison, we will denote objects and functors corresponding on the infinity categorical side to objects of the same nature as those introduced in the previous sections by the same name without the decoration $\mathcal{I}$.

Let us then first recall the definition of $B \mathrm{GL}_{1} R$ in the $\infty$-categorical setting. For an $E_{\infty}$-ring spectrum $R$, the invertible $R$-module spectra and their equivalences span a sub- $\infty$-groupoid $\operatorname{Pic}(R) \in \mathcal{S}_{\infty}$ in the $\infty$-category of all $R$-module spectra. This groupoid inherits a symmetric monoidal structure from the tensor product of $R$-modules, making it an $E_{\infty}$-space with unit $R$. The component of the unit $R$ is usually denoted by $\operatorname{Pic}_{0}(R)$ and as a mere space is clearly also defined for an $E_{1}$-ring spectrum $R$.

LEMMA 10.13. Let $R$ be an $E_{1}$ (respectively $E_{\infty}$ ) ring spectrum and let $\left(\Omega^{\infty} R\right)^{\times}$ be the subspace of $\Omega^{\infty} R$ corresponding to the units in the multiplicative $E_{1}$ (respectively $E_{\infty}$ ) structure. Then there are canonical equivalences

$$
\operatorname{Pic}_{0}(R) \simeq B \operatorname{Aut}_{R}(R) \simeq B\left(\Omega^{\infty} R\right)^{\times}
$$

in $\mathcal{S}_{\infty}$ (respectively $\operatorname{Alg}_{E_{\infty}}\left(\mathcal{S}_{\infty}\right)$ ).

We shall refer to any of these equivalent spaces as $B \mathrm{GL}_{1} R$.

Proof. By definition of mapping space, there is, for every pair of objects $x, y$ of an $\infty$-category $\mathcal{C}$, a cartesian diagram in $\mathrm{Cat}_{\infty}$

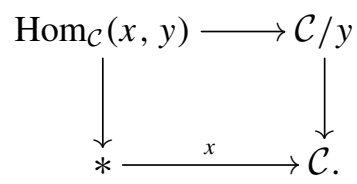

If now $\mathcal{C} \in \mathcal{S}_{\infty}$ is an $\infty$-groupoid and $x=y, \mathcal{C} / y$ is a contractible $\infty$-groupoid and we obtain an equivalence $\Omega_{x} \mathcal{C} \simeq \operatorname{Aut}_{\mathcal{C}}(x)$ and consequently $\mathcal{C}_{x} \simeq B \operatorname{Aut}_{\mathcal{C}}(x)$, where $\mathcal{C}_{x}$ denotes the path component of $x$ in $\mathcal{C}$. Furthermore, if $\mathcal{C}$ is symmetric monoidal and $x$ its unit, then $\mathcal{C} / x$ inherits a symmetric monoidal structure from $\mathcal{C}$, and the diagram defines an $E_{\infty}$-structure on $\operatorname{Aut}_{\mathcal{C}}(x)$ and then becomes cartesian in $\operatorname{Alg}_{E_{\infty}}\left(\mathcal{S}_{\infty}\right)$. Applied to $\mathcal{C}=\operatorname{Pic}_{0}(R)$ and $x=R$, we obtain the first desired equivalence.

For the second, we have to distinguish the two cases: If $R$ is an $E_{\infty}$-ring spectrum, it arises from the adjunction equivalence

$$
\Omega^{\infty}(R) \simeq \operatorname{Hom}_{\mathbb{S}}(\mathbb{S}, R) \stackrel{-\wedge R}{\longrightarrow} \operatorname{Hom}_{R}(R, R \wedge R) \stackrel{\mu}{\rightarrow} \operatorname{End}_{R}(R)
$$


since the functor $-\wedge R$ is symmetric monoidal and the multiplication of $R$ is an $E_{\infty}$-map.

In the case of an $E_{1}$-ring spectrum $R$, the middle term does not carry an evident multiplication, so we have to argue differently. We can obtain a map of $E_{1}$-ring spectra $R \rightarrow \operatorname{end}_{R}(R)$ from [35, Corollary 4.7.1.41 and Remark 7.1.2.2]; here, we apply the corollary to $R$ considered as a left $R$-module spectrum in the $\infty$ category of right $R$-module spectra, which is tensored over and consequently enriched in the $\infty$-category of spectra (see [35, Proposition 4.2.1.33 and Remark 4.8.2.20]). Applying the (symmetric) monoidal functor $\Omega^{\infty} \simeq \operatorname{Hom}_{\mathbb{S}}(\mathbb{S},-$ ) to the above arrow produces a map $\Omega^{\infty} R \rightarrow \operatorname{End}_{R}(R)$ of $E_{1}$-spaces, which equals the above composite and is therefore an equivalence.

It remains to check that the $E_{1}$-structure constructed on $\operatorname{Aut}_{R}(R)$ above via the identification with $\Omega \mathrm{Pic}_{0}(R)$ agrees with the restriction of that just constructed on $\operatorname{End}_{R}(R)$ to its units. But this is clear since lifts of the functor $\Omega: * / \mathcal{S}_{\infty}^{\geqslant 1} \rightarrow \mathcal{S}_{\infty}$ to $E_{1}$-monoids correspond to comonoid structures on $S^{1}$. Under the equivalence of $* / \mathcal{S}_{\infty} \geqslant 1$ with $E_{1}$-groups, these correspond to cogroup structures on the integers, of which there are only two, corresponding to forwards and backwards concatenation of loops (and the above constructions evidently give the same concatenation map up to homotopy).

REMARK 10.14. For an $E_{n}$-ring spectrum with $n \geqslant 2$, the category of left $R$ modules is $E_{n-1}$-monoidal, so $\operatorname{Pic}(R)$ is an $E_{n-1}$-space in this case, as is $B \Omega^{\infty} R$. The method we gave for the $E_{\infty}$-case identifies these as $E_{n-2}$-spaces and one can check that the argument we gave for $E_{1}$-ring spectra identifies the remaining $E_{1}$ structures in a compatible fashion. In total, one thus obtains an identification as $E_{n-2} \otimes E_{1} \simeq E_{n-1}$-spaces.

The space $B \mathrm{GL}_{1} R$ comes equipped with the canonical functor

$$
F_{R}: B \mathrm{GL}_{1} R \simeq \operatorname{Pic}_{0}(R) \longrightarrow R-\operatorname{Mod} \longrightarrow \mathrm{Sp}_{\infty},
$$

which witnesses the action of $\mathrm{GL}_{1} R$ on $R$.

PROPOSITION 10.15. For any symmetric ring spectrum $R$, the equivalence from Proposition 10.4 carries $\gamma_{R} \in \mathrm{Sp}_{\mathcal{R}}^{\Sigma}$ to the image under the inclusion

$$
\operatorname{Fun}\left(B \mathrm{GL}_{1} R, \mathrm{Sp}_{\infty}\right) \longrightarrow \int_{\mathcal{S}_{\infty}} \operatorname{Fun}\left(-, \mathrm{Sp}_{\infty}\right)=T \mathcal{S}_{\infty}
$$

of the functor $F_{R}$. If $R$ is commutative, then $F_{R}$ is a lax symmetric monoidal functor, and the same is true as $E_{\infty}$-monoid objects of $T \mathcal{S}_{\infty}$. 
As preparation, we record the following.

LEMMA 10.16. Let $R$ be a positive fibrant symmetric ring spectrum and $G$ a cofibrant replacement of $\mathrm{GL}_{1}^{\mathcal{I}} R$. Then the image of $B G$ under the equivalence $\mathcal{S}_{\infty}^{\mathcal{I}} \simeq \mathcal{S}_{\infty}$ is $B \mathrm{GL}_{1} R$ and the evaluation map $\mathbb{S}[G] \rightarrow R$ corresponds to the canonical map $\mathbb{S}\left[\mathrm{GL}_{1} R\right] \rightarrow R$. When $R$ is commutative, the same identifications hold in the $\infty$-categories $\left(\mathcal{C S}^{\mathcal{I}}\right)_{\infty} \simeq \operatorname{Alg}_{E_{\infty}}\left(\mathcal{S}_{\infty}\right)$ and $\left(\mathcal{C S p}^{\Sigma}\right)_{\infty} \simeq \operatorname{Alg}_{E_{\infty}}\left(\operatorname{Sp}_{\infty}\right)$.

Proof. All of the assertions follow immediately from the previous lemma and the commutative square

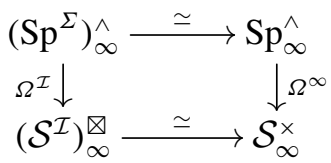

of symmetric monoidal $\infty$-categories and symmetric monoidal functors (lax in the case of the vertical functors): By the monoidality of the horizontal functors, the objects $\Omega^{\mathcal{I}} R$ and $\Omega^{\infty} R$ correspond as $E_{1} / E_{\infty}$ objects and the units are given by the same restriction to path components. The respective bar constructions then agree as simplicial objects by the cofibrancy assumption on $G$ and Hinich's result on the strong monoidality on cofibrant objects of the localization functor from a monoidal model category to its underlying $\infty$-category; see [22, Proposition 3.2.2] and also [40, Appendix A]. Finally, geometric realization in a simplicial model category models the colimit in its underlying $\infty$-category by [34, Theorem 4.2 .4 .1$]$, which gives the claim about $B \mathrm{GL}_{1} R$.

The maps from the spherical group rings to $R$ are the counits of the vertical adjunction with the suspension functor, and so also correspond under the above equivalences.

Proof of Proposition 10.15. The idea is to specify both $\gamma_{R}$ and $F_{R}$ in terms of data pinned down by Theorem 10.10 and the previous lemma. Namely, we will show that both objects are given by the relative $\bar{\wedge}$-product of the diagram $\mathbb{S} \leftarrow \mathbb{S}_{t}\left[\mathrm{GL}_{1} R\right] \rightarrow R$ (with the appropriate interpretations in $\left(\mathrm{Sp}_{\mathcal{R}}^{\Sigma}\right)_{\infty}$ and $T \mathcal{S}_{\infty}$, respectively). Note that this really is determined by the lemma since the map $\mathbb{S}_{t}\left[\mathrm{GL}_{1} R\right] \rightarrow R$ factors by definition (as an $E_{1} / E_{\infty}$-map as appropriate) through the tautological map $\mathbb{S}_{t}\left[\mathrm{GL}_{1} R\right] \rightarrow \mathbb{S}\left[\mathrm{GL}_{1} R\right]$. For $\gamma_{R} \in\left(\mathrm{Sp}_{\mathcal{R}}^{\Sigma}\right)_{\infty}$, the identification with the relative $\bar{\wedge}$-product holds by [35, Theorem 4.4.2.8(ii)] since relative tensor products are computed by the bar construction.

In case $R$ is commutative, we first note that [35, Example 3.2.4.4, Proposition 3.2.4.7 and Theorem 4.5.2.1] together say that for an $E_{\infty}$-ring spectrum $A$, the 
coproduct of $E_{\infty}$-algebras in $A$-modules is given by the relative tensor product. Combining this with the facts that $E_{\infty}$-algebras in $A$-modules are the same thing as $E_{\infty}$-algebras under $A$ [35, Corollary 3.4.1.7] and that coproducts in slice categories are computed as pushouts in the original category, it follows that the above relative tensor product inherits an $E_{\infty}$-structure which makes it the pushout of $E_{\infty}$-rings. In particular, this structure is again determined by data preserved under the equivalence of Theorem 10.10. By applying [35, Theorem 4.4.2.8] to both the category of parametrized spectra and $E_{\infty}$ algebras therein, we find that the $E_{\infty}$ structure on the relative tensor product agrees with that coming from the termwise one on the bar construction (the forgetful functor from $E_{\infty}$ algebras commutes with geometric realization by [35, Proposition 3.2.3.1]). We therefore find that the $E_{\infty}$ structure on $\gamma_{R} \in\left(\operatorname{Sp}_{\mathcal{R}}^{\Sigma}\right)_{\infty}$ agrees with that on the relative tensor product.

For the case of $F_{R} \in T S_{\infty}$, we argue by identifying both $F_{R}$ and the relative $\bar{\wedge}$-product as colimits of the functor

$$
G_{R}: B \mathrm{GL}_{1} R \stackrel{F_{R}}{\longrightarrow} \mathrm{Sp}_{\infty} \longrightarrow T \mathcal{S}_{\infty}
$$

whose second part is the inclusion over the one point space. If $R$ is commutative, it will be an identification as $E_{\infty}$-algebras. This has meaning since $F_{R}$ and thus $G_{R}$ are lax symmetric monoidal in this case, whence the colimit of $G_{R}$ inherits an $E_{\infty}$-structure, for example, by [38, Proposition 3.3 and Corollary 3.8].

To see that colim $G_{R} \simeq F_{R}$, we employ one direction of [35, Proposition 7.3.1.12(1)]. Informally speaking, it says that

$$
\operatorname{colim}_{b \in B \mathrm{GL}_{1} R} F_{R}(b) \simeq \operatorname{colim}_{b \in B \mathrm{Gl}_{1} R}\left(\iota_{b}\right) ! F_{R}(b),
$$

where $\iota_{b}: * \rightarrow B \mathrm{GL}_{1} R$ denotes the inclusion of $b$. Formally, the right-hand side arises as follows: Choose a colimit extension of $G_{R}$ to the cone category $B \mathrm{GL}_{1} R^{\triangleright}$ and consider the lifting problem consisting of the solid parts of

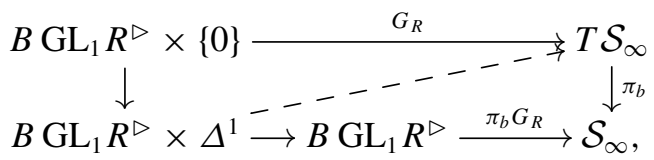

using the map $B \mathrm{GL}_{1} R^{\triangleright} \times \Delta^{1} \rightarrow B \mathrm{GL}_{1} R^{\triangleright}$ that is the evident projection on $B \mathrm{GL}_{1} R^{\triangleright} \times\{0\}$ and collapses $B \mathrm{GL}_{1} R^{\triangleright} \times\{1\}$ to the cone point. By (the duals of) [34, Remark 2.4.1.9 and Propositions 3.1.2.1], there exists an essentially unique diagonal filler mapping every edge $\{b\} \times \Delta^{1}$ to a cocartesian edge since $\pi_{b}$ is cocartesian. The restriction $H_{R}$ of this filler to $B \mathrm{GL}_{1} R \times\{1\}$ canonically factors through the inclusion $\operatorname{Fun}\left(B \mathrm{GL}_{1} R, \mathrm{Sp}_{\infty}\right) \rightarrow T \mathcal{S}_{\infty}$ since $\pi_{b} \circ H_{R}$ is 
the constant functor with value $B \mathrm{GL}_{1} R$ by the reverse implication of [35, Proposition 7.3.1.12(1)]. Regarded as a functor $H_{R}: B \mathrm{GL}_{1} R \rightarrow \operatorname{Fun}\left(B \mathrm{GL}_{1} R\right.$, $\mathrm{Sp}_{\infty}$ ), the $H_{R}$ then defines the right-hand side in the original assertion (10.17) and the agreement of the two colimits is an instance of [34, Proposition 4.3.1.10]. Now $H_{R}$ is adjoint to $\Delta_{!} F_{R}: B \mathrm{GL}_{1} R \times B \mathrm{GL}_{1} R \rightarrow \mathrm{Sp}_{\infty}$ : The value of the adjoint of $H_{R}$ on a pair of objects $\left(b, b^{\prime}\right) \in B \mathrm{GL}_{1} R \times B \mathrm{GL}_{1} R$ is by construction the colimit of the functor

$$
\iota_{b} / b^{\prime} \longrightarrow\{b\} \stackrel{F_{R}}{\longrightarrow} \mathrm{Sp}_{\infty}
$$

where $\iota_{b}: * \rightarrow B \mathrm{GL}_{1} R$ denotes the inclusion of $b$ again, whereas the left Kan extension along $\Delta: B \mathrm{GL}_{1} R \rightarrow B \mathrm{GL}_{1} R \times B \mathrm{GL}_{1} R$ by definition evaluates to the colimit of

$$
\Delta /\left(b, b^{\prime}\right) \longrightarrow B \mathrm{GL}_{1} R \stackrel{F_{R}}{\longrightarrow} \mathrm{Sp}_{\infty} .
$$

But taking products with $\mathrm{id}_{b}$ induces an equivalence $\iota_{b} / b^{\prime} \rightarrow \Delta /\left(b, b^{\prime}\right)$ making the above triangle commute, from which the claim follows by the uniqueness of Kan extensions. We thus find

$$
\begin{aligned}
\operatorname{Hom}_{B \mathrm{GL}_{1} R}\left(\operatorname{colim} H_{R},-\right) & \simeq \operatorname{Hom}_{B \mathrm{GL}_{1} R \times B \mathrm{GL}_{1} R}\left(\Delta_{!} F_{R}, \mathrm{pr}_{2}^{*}-\right) \\
& \simeq \operatorname{Hom}_{B \mathrm{GL}_{1} R}\left(F_{R},-\right)
\end{aligned}
$$

as functors on $\operatorname{Fun}\left(B \mathrm{GL}_{1} R, \mathrm{Sp}_{\infty}\right)$, the first by definition and the second by the adjunction $\left(\Delta_{!}, \Delta^{*}\right)$. It follows that colim $G_{R} \simeq \operatorname{colim} H_{R} \simeq F_{R}$ as desired.

For $R$ commutative, the functor $G_{R}$ extends to a map $G_{R}^{\otimes}:\left(B \mathrm{GL}_{1} R\right)^{\wedge} \rightarrow$ $(T S)^{\wedge}$ witnessing the lax monoidality of $G_{R}$. Repeating the same argument then shows that, in this case, colim $G_{R} \simeq F_{R}$ as lax symmetric monoidal functors.

To see that the relative $\bar{\wedge}$-product is also a colimit of $G_{R}$, we follow the proof of [2, Proposition 3.26]: They consider the inclusion of the category $B \mathrm{GL}_{1} R$ into the category Fun $\left(B \mathrm{GL}_{1} R, \mathcal{S}_{\infty}\right)$ of all $\mathrm{GL}_{1} R$-spaces as the automorphisms of $\mathrm{GL}_{1} R$. Then the diagram

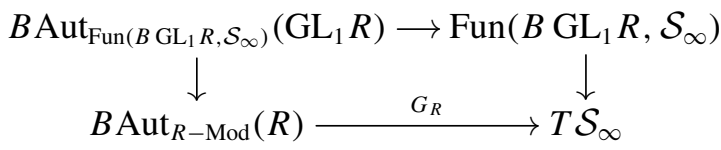

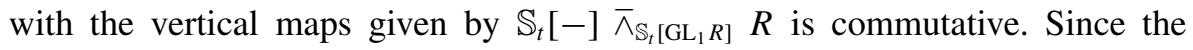
left vertical map is an equivalence of $\infty$-groupoids, the colimit over $G_{R}$ may equally well be computed using the upper composite. Since the right vertical map preserves colimits, this amounts to computing the relative $\bar{\wedge}$-product of $R$ with the suspension spectrum of the colimit of the inclusion

$$
B \operatorname{Aut}_{\mathrm{Fun}\left(B \mathrm{GL}_{1} R, \mathcal{S}_{\infty}\right)}\left(\mathrm{GL}_{1} R\right) \rightarrow \operatorname{Fun}\left(B \mathrm{GL}_{1} R, \mathcal{S}_{\infty}\right)
$$


This is the terminal object by the argument preceding [2, Proposition 3.26], and we obtain the desired identification.

In case $R$ is commutative, all functors in sight are lax symmetric monoidal (for the Day convolution on the upper right corner), whence the identification of colimits preserves $E_{\infty}$-structures.

We can now also compare our definition of twisted (co)homology from Section 7 with the $\infty$-categorical one given in $[1,2]$.

Proposition 10.18. Let $R$ be a positive fibrant symmetric ring spectrum, let $G \rightarrow \mathrm{GL}_{1}^{\mathcal{I}}(R)$ be a cofibrant replacement, and let $\left(\gamma_{R}, B G\right)$ be the universal line bundle. Given a map $\tau: K \rightarrow B G_{h \mathcal{I}}$, there are canonical isomorphisms relating our $\left(\gamma_{R}, B G\right)_{n}(K, \tau)$ and $\left(\gamma_{R}, B G\right)^{n}(K, \tau)$ with [1, Definition 1.2] applied to

$$
K \stackrel{\tau}{\rightarrow}\{n\} \times B \mathrm{GL}_{1} R \rightarrow \operatorname{Pic}(R) .
$$

In the formulation, we have again used Lemma 10.16 to identify $B G_{h \mathcal{I}}$ with the $\infty$-categorical $B \mathrm{GL}_{1} R$.

Proof. By the suspension isomorphism, it suffices to consider the case $n=0$. Let us then recall the relevant definitions (in our notation) for $\sigma: L \rightarrow B \mathrm{GL}_{1} R$ :

$$
R^{0}(L, \sigma)=\pi_{0} F^{R}\left(\Theta \check{\sigma}^{*} \gamma_{R}, R\right) \quad \text { and } \quad R_{0}(L, \sigma)=\pi_{0} F^{R}\left(R, \Theta \sigma^{*} \gamma_{R}\right),
$$

where $F^{R}$ denotes the spectrum of $R$-linear maps and $\check{\sigma}$ denotes the composite

$$
L \stackrel{\sigma}{\rightarrow} B \mathrm{GL}_{1} R \stackrel{(-)^{-1}}{\longrightarrow} B \mathrm{GL}_{1} R
$$

The functor $\Theta$ being left adjoint to pullback along the constant map makes it the $\infty$-categorical colimit. In the $\infty$-category of spectra, we then have

$$
F^{R}\left(\Theta \check{\sigma}^{*} \gamma_{R}, R\right) \simeq F_{L}^{R}\left(\check{\sigma}^{*} \gamma_{R}, R_{L}\right) \simeq F_{L}^{R}\left(R_{L}, \sigma^{*} \gamma_{R}\right) \simeq F^{R}\left(R, \Gamma \sigma^{*} \gamma_{R}\right),
$$

where $(-)_{L}$ denotes the constant diagram functor $\operatorname{Sp}_{\infty} \rightarrow \operatorname{Fun}\left(L, \operatorname{Sp}_{\infty}\right)$, and the second equivalence is given by fiberwise smashing (over $R$ and $L$ ) with $\sigma^{*} \gamma_{R}$ and the remainder by the definition of $\Theta$ and $\Gamma$ as adjoints to $(-)_{L}$. Identifying $F^{R}(R,-)$ with the identity functor on $R$-module spectra, we therefore obtain from Lemma 10.3 and Proposition 10.15 that both the definitions of homology and cohomology agree with ours.

Finally, we record the agreement of our products (7.13) with their $\infty$ categorical counterparts. 
Proposition 10.19. Let $R$ be a positive fibrant commutative symmetric ring spectrum. Then the products (7.13) agree with those from [1, Theorem 4.21] under the isomorphisms from Proposition 10.18.

Proof. This follows immediately from the conjunction of Theorem 10.6, Proposition 10.16, and Proposition 10.15 by unwinding the definitions.

\section{Acknowledgements}

The authors would like to thank Samik Basu, Emanuele Dotto, Gijs Heuts, Michael Joachim, Cary Malkiewich, Irakli Patchkoria, and Tomer Schlank for useful conversations and particularly Thomas Nikolaus for help with the final chapter. The first author held a scholarship of the German Academic Exchange Service during a year at the University of Notre Dame, when initial work on this project was undertaken. The third author was supported by Meltzers Høyskolefond. Furthermore, the authors would like to thank the Isaac Newton Institute for Mathematical Sciences for support and hospitality during the program 'Homotopy harnessing higher structures'. This work was supported by EPSRC grants EP/K032208/1 and EP/R014604/1 and the Hausdorff Center for Mathematics, DFG GZ 2047/1, project ID 390685813.

Conflict of Interest: There are no conflicts of interest.

\section{References}

[1] M. Ando, A. J. Blumberg and D. Gepner, 'Parametrized spectra, multiplicative Thom spectra, and the twisted Umkehr map', Geom. Topol. 22 (2018), 3761-3825.

[2] M. Ando, A. J. Blumberg, D. Gepner, M. J. Hopkins and C. Rezk, 'An $\infty$-categorical approach to $R$-line bundles, $R$-module Thom spectra, and twisted $R$-homology', J. Topol. 7(3) (2014), 869-893.

[3] M. Ando, A. J. Blumberg, D. Gepner, M. J. Hopkins and C. Rezk, 'Units of ring spectra, orientations and Thom spectra via rigid infinite loop space theory', J. Topol. 7(4) (2014), 1077-1117.

[4] B. Antieau, D. Gepner and J. M. Gómez, 'Actions of $K(\pi, n)$ spaces on $K$-theory and uniqueness of twisted $K$-theory', Trans. Amer. Math. Soc. 366(7) (2014), 3631-3648.

[5] C. Barwick, 'On left and right model categories and left and right Bousfield localizations', Homology Homotopy Appl. 12(2) (2010), 245-320.

[6] S. Basu, S. Sagave and C. Schlichtkrull, 'Generalized Thom spectra and their topological Hochschild homology', J. Inst. Math. Jussieu 19(1) (2020), 21-64.

[7] A. J. Blumberg, R. L. Cohen and C. Schlichtkrull, 'Topological Hochschild homology of Thom spectra and the free loop space', Geom. Topol. 14(2) (2010), 1165-1242.

[8] F. Borceux, Handbook of Categorical Algebra. 1, Encyclopedia of Mathematics and its Applications, 50 (Cambridge University Press, Cambridge, 1994). 
[9] F. Borceux, Handbook of Categorical Algebra. 2, Encyclopedia of Mathematics and its Applications, 51 (Cambridge University Press, Cambridge, 1994).

[10] A. K. Bousfield, 'On the telescopic homotopy theory of spaces', Trans. Amer. Math. Soc. 353(6) (2001), 2391-2426.

[11] V. S. Braunack-Mayer, 'Rational parametrised stable homotopy theory', PhD Thesis, University of Zurich, 2018, available at https://doi.org/10.5167/uzh-153000.

[12] P. Cagne and P.-A. Melliès, 'On bifibrations of model categories'. Preprint, 2017, arXiv:1709.10484.

[13] M. Clapp and D. Puppe, 'The homotopy category of parametrized spectra', Manuscripta Math. 45(3) (1984), 219-247.

[14] D. Dugger, 'Replacing model categories with simplicial ones', Trans. Amer. Math. Soc. 353(12) (2001), 5003-5027.

[15] D. Gepner, M. Groth and T. Nikolaus, 'Universality of multiplicative infinite loop space machines', Algebr. Geom. Topol. 15(6) (2015), 3107-3153.

[16] S. Glasman, 'Day convolution for $\infty$-categories', Math. Res. Lett. 23(5) (2016), 1369-1385.

[17] P. G. Goerss and J. F. Jardine, Simplicial Homotopy Theory, Progress in Mathematics, 174 (Birkhäuser, Basel, 1999).

[18] S. Gorchinskiy and V. Guletskiǔ, 'Positive model structures for abstract symmetric spectra', Appl. Categ. Structures 26(1) (2018), 29-46.

[19] Y. Harpaz, J. Nuiten and M. Prasma, 'The tangent bundle of a model category', Theory Appl. Categ. 34 (2019), 1039-1072, Paper No. 33.

[20] Y. Harpaz and M. Prasma, 'The Grothendieck construction for model categories', Adv. Maths 281 (2015), 1306-1363.

[21] F. Hebestreit and S. Sagave, 'Homotopical and operator algebraic twisted $K$-theory', Preprint, 2019, arXiv:1904.01872v1.

[22] V. Hinich, 'Dwyer-Kan localization revisited', Homology Homotopy Appl. 18(1) (2016), 27-48.

[23] P. S. Hirschhorn, Model Categories and Their Localizations, Mathematical Surveys and Monographs, 99 (American Mathematical Society, Providence, RI, 2003).

[24] P. S. Hirschhorn, 'Overcategories and undercategories of model categories', Preprint, 2015, arXiv:1507.01624.

[25] M. Hovey, Model Categories, Mathematical Surveys and Monographs, 63 (American Mathematical Society, Providence, RI, 1999).

[26] M. Hovey, 'Spectra and symmetric spectra in general model categories', J. Pure Appl. Algebra 165(1) (2001), 63-127.

[27] M. Hovey, B. Shipley and J. Smith, 'Symmetric spectra', J. Amer. Math. Soc. 13(1) (2000), 149-208.

[28] M. Joachim, 'Higher coherences for equivariant $K$-theory', in Structured Ring Spectra, London Mathematical Society Lecture Note Series, 315 (Cambridge University Press, Cambridge, 2004), 87-114.

[29] A. Joyal, 'The theory of quasi-categories and its applications', 2008. Available at http://mat.uab.cat/ kock/crm/hocat/advanced-course/Quadern45-2.pdf.

[30] L. G. Lewis Jr., 'The stable category and generalized Thom spectra', PhD Thesis, University of Chicago, 1978.

[31] L. G. Lewis Jr., 'When is the natural map $X \rightarrow \Omega \Sigma X$ a cofibration?', Trans. Amer. Math. Soc. 273(1) (1982), 147-155.

[32] L. G. Lewis Jr., 'Open maps, colimits, and a convenient category of fibre spaces', Topology Appl. 19(1) (1985), 75-89. 
[33] L. G. Lewis Jr., J. P. May, M. Steinberger and J. E. McClure, Equivariant Stable Homotopy Theory, Lecture Notes in Mathematics, 1213 (Springer, Berlin, 1986), With contributions by J. E. McClure.

[34] J. Lurie, Higher Topos Theory, Annals of Mathematics Studies, 170 (Princeton University Press, Princeton, NJ, 2009).

[35] J. Lurie, 'Higher algebra', Preprint, 2016, available at http://www.math.harvard.edu/ lurie/.

[36] M. A. Mandell, J. P. May, S. Schwede and B. Shipley, 'Model categories of diagram spectra', Proc. Lond. Math. Soc. (3) 82(2) (2001), 441-512.

[37] J. P. May and J. Sigurdsson, Parametrized Homotopy Theory, Mathematical Surveys and Monographs, 132 (American Mathematical Society, Providence, RI, 2006).

[38] T. Nikolaus, 'Stable $\infty$-operads and the multiplicative Yoneda lemma', Preprint, 2016, arXiv:1608.02901.

[39] T. Nikolaus and S. Sagave, 'Presentably symmetric monoidal $\infty$-categories are represented by symmetric monoidal model categories', Algebr. Geom. Topol. 17(5) (2017), 3189-3212.

[40] T. Nikolaus and P. Scholze, 'On topological cyclic homology', Acta Math. 221(2) (2018), 203-409.

[41] D. Pavlov and J. Scholbach, 'Symmetric operads in abstract symmetric spectra', J. Inst. Math. Jussieu 18(4) (2019), 707-758.

[42] S. Sagave and C. Schlichtkrull, 'Diagram spaces and symmetric spectra', Adv. Maths 231(34) (2012), 2116-2193.

[43] S. Sagave and C. Schlichtkrull, 'Group completion and units in $\mathcal{I}$-spaces', Algebr. Geom. Topol. 13(2) (2013), 625-686.

[44] C. Schlichtkrull, 'Units of ring spectra and their traces in algebraic $K$-theory', Geom. Topol. 8 (2004), 645-673 (electronic).

[45] C. Schlichtkrull, 'Thom spectra that are symmetric spectra', Doc. Math. 14 (2009), 699-748.

[46] J. Schulz, 'Logarithmic structures on commutative $H k$-algebra spectra', $\mathrm{PhD}$ Thesis, Universität Hamburg, 2018, available at http://ediss.sub.uni-hamburg.de/volltexte/2018/9312/.

[47] S. Schwede, 'Symmetric spectra', 'Book project', 2012, available at http://www.math.uni-bonn.de/people/schwede/.

[48] S. Schwede and B. E. Shipley, 'Algebras and modules in monoidal model categories', Proc. Lond. Math. Soc. (3) 80(2) (2000), 491-511.

[49] B. Shipley, 'A convenient model category for commutative ring spectra', in Homotopy Theory: Relations with Algebraic Geometry, Group Cohomology, and Algebraic K-theory, Contemporary Mathematics, 346 (American Mathematical Society, Providence, RI, 2004), 473-483.

[50] R. W. Thomason, 'Homotopy colimits in the category of small categories', Math. Proc. Cambridge Philos. Soc. 85(1) (1979), 91-109. 